\title{
Grazoprevir plus elbasvir in treatment-naive and treatment-experienced patients with hepatitis $C$ virus genotype 1 infection and stage 4-5 chronic kidney disease (the C-SURFER study): a combination phase 3 study
}

\author{
David Roth, David R Nelson, Annette Bruchfeld, AnnMarie Liapakis, Marcelo Silva, Howard Monsour Jr, Paul Martin, Stanislas Pol, \\ Maria-Carlota Londoño, Tarek Hassanein, Philippe J Zamor, Eli Zuckerman, Shuyan Wan, Beth Jackson, Bach-Yen Nguyen, Michael Robertson, \\ Eliav Barr, Janice Wahl, Wayne Greaves
}

\section{Summary}

Background Chronic hepatitis C virus (HCV) infection in patients with stage $\mathbf{4} \mathbf{- 5}$ chronic kidney disease increases the risk of death and renal graft failure, yet patients with hepatitis $\mathrm{C}$ and chronic kidney disease have few treatment options. This study assesses an all-oral, ribavirin-free regimen in patients with HCV genotype 1 infection and stage 4-5 chronic kidney disease.

Methods In this phase 3 randomised study of safety and observational study of efficacy, patients with HCV genotype 1 infection and chronic kidney disease (stage 4-5 with or without haemodialysis dependence) were randomly assigned to receive grazoprevir (100 mg, NS3/4A protease inhibitor) and elbasvir (50 mg, NS5A inhibitor; immediate treatment group) or placebo (deferred treatment group) once daily for 12 weeks. Randomisation was done centrally with an interactive voice response system. An additional cohort of patients who were not randomised received the same regimen open-label and underwent intensive pharmacokinetic sampling. The primary efficacy outcome was a nonrandomised comparison of sustained virological response at 12 weeks (SVR12) after the end of therapy for the combined immediate treatment group and the pharmacokinetic population with a historical control. The primary safety outcome was a randomised comparison between the immediate treatment group and the deferred treatment group. After 4 weeks of follow-up (study week 16), unmasking occurred and patients in the deferred treatment group received grazoprevir and elbasvir. The primary efficacy hypothesis was tested at a two-sided significance level (type I error) of $\mathbf{0 . 0 5}$ using an exact test for a binomial proportion. Safety event rates were compared between immediate treatment and deferred treatment groups using the stratified Miettinen and Nurminen method with baseline dialysis status as the strata. The study is registered at ClinicalTrials.gov, number NCT02092350.

Findings 224 patients were randomly assigned to the immediate treatment group with grazoprevir and elbasvir $(n=111)$ or the deferred treatment group $(n=113)$, and 11 were assigned to the intensive pharmacokinetic population. Overall, 179 (76\%) were haemodialysis-dependent, 122 (52\%) had HCV genotype 1a infection, 189 (80\%) were HCV treatment-naive, 14 (6\%) were cirrhotic, and 108 (46\%) were African American. Of the 122 patients receiving grazoprevir and elbasvir, six were excluded from the primary efficacy analysis for non-virological reasons (death, lost-to-follow-up [n=2], non-compliance, patient withdrawal, and withdrawal by physician for violent behaviour). No patients in the combined immediate treatment group and intensive pharmacokinetic population and five $(4 \%)$ in the deferred treatment group discontinued because of an adverse event. Most common adverse events were headache, nausea, and fatigue, occurring at similar frequencies in patients receiving active and placebo drugs. SVR12 in the combined immediate treatment group and intensive pharmacokinetic population was $99 \%$ (95\% CI $95 \cdot 3-100 \cdot 0 ; 115 / 116)$, with one relapse 12 weeks after end of treatment when compared with a historical control of $45 \%$, based on meta-analyses of interferon-based regimens used in clinical trials of patients infected with $\mathrm{HCV}$ who are on haemodialysis.

Interpretation Once-daily grazoprevir and elbasvir for 12 weeks had a low rate of adverse events and was effective in patients infected with HCV genotype 1 and stage 4-5 chronic kidney disease.

Funding Merck Sharp \& Dohme Corp.

\section{Introduction}

Hepatitis C infection accelerates the decline in kidney function in patients with chronic kidney disease and increases mortality among patients on haemodialysis ${ }^{1}$ compared with patients not infected with hepatitis $\mathrm{C}$ on dialysis..$^{2-6}$ Studies among kidney transplant patients show infection with hepatitis $\mathrm{C}$ also has an adverse effect on patient and graft survival. ${ }^{-9}$ These data suggest that clearance of hepatitis $\mathrm{C}$ infection among patients with stage 4-5 chronic kidney disease (estimated glomerular
Published Online October 6, 2015 http://dx.doi.org/10.1016/ S0140-6736(15)00349-9

See Online/Comment http://dx.doi.org/10.1016/ S0140-6736(15)00381-5

Division of Nephrology and Hypertension, University of Miami Miller School of Medicine, Miami, FL, USA (D Roth MD); Clinical and Translational Science Institute, University of Florida, Gainesville, FL, USA (D R Nelson MD); Department of Renal Medicine, Karolinska University Hospital, Karolinska Institute, Stockholm, Sweden (A Bruchfeld MD); Yale University Digestive Disease, Yale New Haven Hospita Transplant Center, New Haven, $\mathrm{CT}$, USA

(A M Liapakis MD); Hospital Universitario Austral, Pilar Argentina (M Silva MD); Hepatology \& Transplant Medicine, Houston Methodist Hospital, Houston, TX, USA (H Monsour Jr MD); Division of Hepatology, University of Miami Miller School of Medicine, Miami, FL, USA (P Martin MD); Unité d'Hépatologie, Hôpital Cochin; Université Paris Descartes; and UMS20, Institut Pasteur; Paris, France (S Pol MD); Liver Unit, Hospital Clinic Barcelona, IDIBAPS, CIBERehd, Barcelona, Spain (M-C Londoño MD); Southern California Research Center, Coronado, CA, USA (T Hassanein MD); Division of Hepatology, Carolinas Medical Center, Charlotte, NC, USA (P) Zamor MD); Liver Unit, Carmel Medical Center Technion Faculty of Medicine, Haifa, Israel (E Zuckerman MD); and Merck \& Co, Inc 


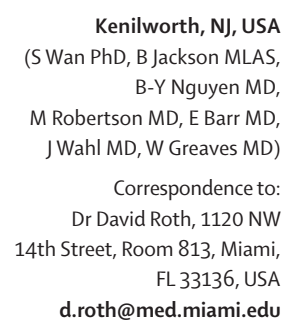

Kenilworth, NJ, USA B Jackson MLAS, B-Y Nguyen MD, Robertson MD, E Barr MD, Correspondence to: Street, Room 813, Miami, d.roth@med.miami.edu

\section{Research in context}

\section{Evidence before this study}

Patients with stage 4-5 chronic kidney disease and hepatitis $C$ infection have few treatment options for hepatitis C virus (HCV). At the time this study was designed, the Kidney Disease: Improving Global Outcomes (KDIGO) recommended treatment was interferon or pegylated interferon. Some investigators also explored adding ribavirin to pegylated interferon. Unfortunately, these regimens are associated with treatment-limiting toxic effects and suboptimum efficacy. We searched PubMed for clinical trials published before Jan 31, 2015, describing the treatment of hepatitis $\mathrm{C}$ in patients with advanced chronic kidney disease. Although this search returned a total of 97 relevant articles, the data on this subject are summarised most concisely in a series of meta-analyses. The most recent of these meta-analyses (based on data from 28 clinical trials done between 1990 and 2006, and including 645 patients) suggests that interferon or pegylated interferon monotherapy was associated with a sustained virological response (SVR) in about one in three patients when treated for 16-48 weeks, whereas about $20-25 \%$ of patients did not complete treatment. In the past 5 years, the introduction of direct-acting antiviral therapies has dramatically improved treatment options for patients with hepatitis C. High rates of SVR, coupled with improved tolerability, are now available to many patients with HCV infection; however, in our literature review, we were unable to identify any published studies of direct-acting antiviral therapies in patients with advanced chronic kidney disease. Thus, patients with hepatitis $C$ and stage 4-5 chronic kidney disease remain underserved by current direct-acting antiviral hepatitis $C$ treatment regimens.

\section{Added value of this study}

This study is the first phase 3 study to assess an interferon-free, ribavirin-free, all-oral treatment regimen for patients with HCV infection and advanced (stage 4-5) chronic kidney disease. Patients receiving grazoprevir plus elbasvir for 12 weeks had a low rate of adverse events compared with a deferred treament group and achieved a 99\% SVR12 compared with a historical control.

\section{Implications of all the available evidence}

Grazoprevir and elbasvir is an investigational medicine and is not approved for the treatment of HCV infection. However, data from the present study suggest that the availability of a grazoprevir and elbasvir regimen for patients with stage 4-5 chronic kidney disease could represent a marked improvement in treatment for this significantly underserved patient group. filtration rate $[\mathrm{eGFR}] \leq 29 \mathrm{~mL} / \mathrm{min}$ per $1.73 \mathrm{~m}^{2}$ or on dialysis), especially those who are candidates for kidney transplantation, is of great importance.

Treatment options for patients with hepatitis C infection and stage 4-5 chronic kidney disease remain suboptimum. Approved all-oral therapies are not ideal regimens because they contain drugs whose metabolites are cleared by the kidney (such as sofosbuvir) or because they need co-administration with ribavirin, which is associated with anaemia.

Grazoprevir, a hepatitis C virus (HCV) NS3/4A protease inhibitor, and elbasvir, an NS5A protein inhibitor, are undergoing clinical assessment as a once-daily regimen for the treatment of HCV genotype 1, 4, and 6 infections. ${ }^{10-14}$ Phase 1 studies have shown that less than $1 \%$ of grazoprevir and elbasvir are renally excreted, and that dose adjustments of grazoprevir or elbasvir are not needed in the setting of non-dialysis-dependent stage 4-5 chronic kidney disease and dialysis-dependent stage 5 chronic kidney disease. ${ }^{15}$ C-SURFER (Hepatitis C: Study to Understand Renal Failure's Effect on Responses) is the first phase 3 study of an all-oral HCV regimen in patients with stage 4-5 chronic kidney disease and HCV genotype 1 infection. The aims of the study were to assess the efficacy, safety, and tolerability of grazoprevir plus elbasvir in patients with HCV genotype 1 infection and with chronic kidney disease stage 4-5.

\section{Methods}

\section{Study design and participants}

C-SURFER is a multicentre, phase 3, double-blind study comprising a randomised study of safety and an observational study of efficacy. Adult patients infected with HCV genotype 1 and with chronic kidney disease (stage 4-5 with or without haemodialysis dependence) were selected for inclusion. Complete eligibility criteria are provided in the study protocol. Chronic kidney disease stages 4 and 5 were defined based on eGFR (according to the Modification of Diet in Renal Disease [MDRD]-4 equation) $)^{16} 15-29 \mathrm{~mL} /$ min per $1.73 \mathrm{~m}^{2}$ and less than $15 \mathrm{~mL} / \mathrm{min}$ per $1.73 \mathrm{~m}^{2}$ or on dialysis, respectively. Patients were either treatmentnaive for HCV or had previously received an interferon regimen. Liver staging was based on biopsy within 24 months of enrolment; Fibroscan within 12 months of enrolment; or a combination of Fibrotest score greater than 0.75 and an AST to platelet ratio of greater than $2 .^{17-19}$

The study was done at 68 centres in the USA, Argentina, Australia, Canada, Estonia, France, Israel, South Korea, Lithuania, Netherlands, Spain, and Sweden in accordance with the Declaration of Helsinki, the International Conference on Harmonization guidelines, and other regulations governing clinical study conduct. The protocol was approved by an independent ethics committee or institutional review board at each participating site. All patients provided written informed consent.

\section{Randomisation and masking}

Participants were randomly assigned (1:1) to receive grazoprevir $100 \mathrm{mg}$ and elbasvir $50 \mathrm{mg}$ once daily (immediate treatment group) or placebo (deferred treatment group) for 12 weeks. 4 weeks after the end of treatment (week 16), patients and site personnel were unmasked, and those randomised to the deferred 
treatment group received grazoprevir $100 \mathrm{mg}$ and elbasvir $50 \mathrm{mg}$ once daily for 12 weeks (appendix). An additional cohort received open-label grazoprevir $100 \mathrm{mg}$ and elbasvir $50 \mathrm{mg}$ once daily for 12 weeks and underwent intensive pharmacokinetic sampling. Patients were recruited on a voluntary basis at study sites with expertise in conducting pharmacokinetic studies.

Randomisation for the safety study was done centrally using an interactive voice response system and stratified according to dialysis (yes/no) and presence of diabetes (yes/no) with a block size of 4. Grazoprevir, elbasvir, and placebos were manufactured to preserve masking (confirmed as visually identical) and packaged identically. All clinical supplies were provided by Merck \& Co., Inc. Patients, investigators, and site personnel were masked to treatment assignment.

\section{Procedures}

Blood samples for assessment of HCV RNA were collected at baseline, at treatment weeks 1, 2, 3, 4, 6, 8, 10, and 12 , and at 4,12 , and 24 weeks after end of treatment. Plasma HCV RNA concentrations were measured using
Roche COBAS Ampliprep/COBAS Taqman HCV test v2.0 (Roche, Indianapolis, IN, USA) with a lower limit of see Online for appendix quantification of less than $15 \mathrm{IU} / \mathrm{mL}$. Blood samples for assessment of viral resistance were collected at baseline from all patients, and at virological failure for patients with HCV RNA greater than $1000 \mathrm{IU} / \mathrm{mL}$ who met criteria for virological failure. For patients on haemodialysis, laboratory sampling was done before dialysis.

Patients underwent routine laboratory testing, electrocardiograms, and symptom-directed physical examinations at baseline, and during, and after completion of treatment. Adverse events were graded according to a standardised scale (study protocol, appendix).

The deferred treatment group served as an internal control for potential safety signals in the immediate treatment group. Active therapy in the deferred treatment group is ongoing: data described herein are observations from the initial placebo treatment period plus 14 days (results of the deferred open-label treatment with grazoprevir and elbasvir will be presented elsewhere). All patients will be followed for 24 weeks after completion of therapy.

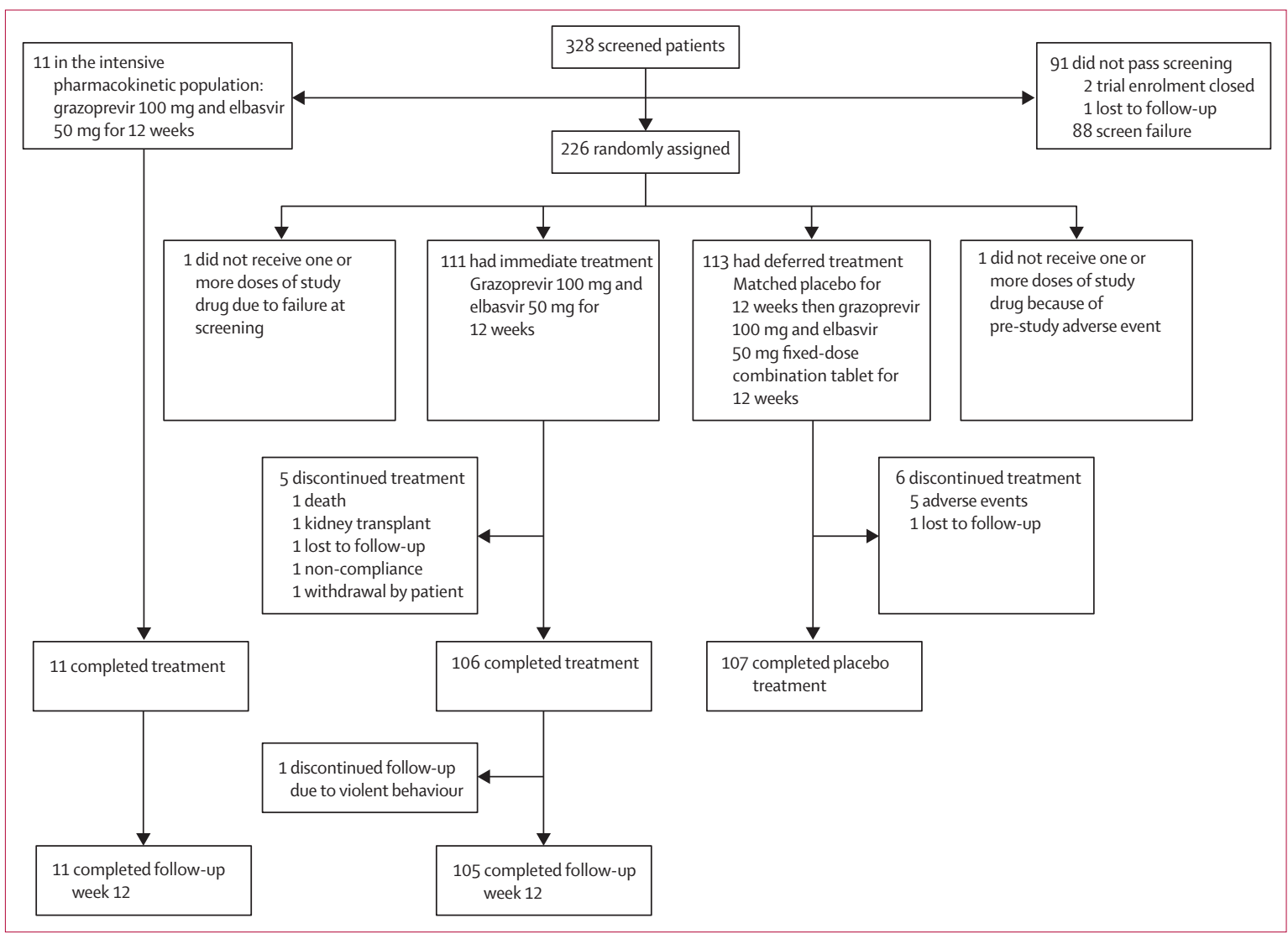

Figure 1: Trial profile

For the deferred treatment group, this figure does not show two deaths (pneumonia and unknown cause of death) that occurred after completion of treatment. In total, there were three deaths in the deferred treatment group (aortic aneurysm, pneumonia, and unknown cause of death): one patient discontinued due to an adverse event and then died (listed as discontinued). One patient in the immediate-treatment group discontinued study drug due to a kidney transplant at treatment week 4 but was not excluded from the modified full analysis set population because the patient continued to participate in the study, remaining in follow-up despite early discontinuation of the study drug. 


\begin{tabular}{|c|c|c|c|c|}
\hline & $\begin{array}{l}\text { Grazoprevir and } \\
\text { elbasvir } \\
\text { pharmacokinetic } \\
\text { population }(n=11)\end{array}$ & $\begin{array}{l}\text { Grazoprevir and } \\
\text { elbasvir immediate } \\
\text { treatment group } \\
(n=111)\end{array}$ & $\begin{array}{l}\text { Grazoprevir and } \\
\text { elbasvir deferred } \\
\text { treatment group } \\
(n=113)\end{array}$ & Total $(\mathrm{n}=235)$ \\
\hline \multicolumn{5}{|l|}{ Sex } \\
\hline Male & $11(100 \%)$ & $81(73.0 \%)$ & $80(70 \cdot 8 \%)$ & $172(73 \cdot 2 \%)$ \\
\hline Female & 0 & $30(27 \cdot 0 \%)$ & $33(29 \cdot 2 \%)$ & $63(26.8 \%)$ \\
\hline Age, years & $58 \cdot 2(6 \cdot 8)$ & $56 \cdot 5(9 \cdot 1)$ & $55 \cdot 2(10 \cdot 1)$ & $56.0(9.5)$ \\
\hline \multicolumn{5}{|l|}{ Race } \\
\hline White & $6(54 \cdot 5 \%)$ & $55(49 \cdot 5 \%)$ & $48(42.5 \%)$ & $109(46 \cdot 4 \%)$ \\
\hline African-American & $5(45 \cdot 5 \%)$ & $50(45 \cdot 0 \%)$ & $53(46 \cdot 9 \%)$ & $108(46 \cdot 0 \%)$ \\
\hline Asian & 0 & $5(4 \cdot 5 \%)$ & $9(8.0 \%)$ & $14(6 \cdot 0 \%)$ \\
\hline Other & 0 & $1(0.9 \%)$ & $3(2 \cdot 7 \%)$ & $4(1 \cdot 7 \%)$ \\
\hline \multicolumn{5}{|l|}{ Ethnic origin } \\
\hline Hispanic-Latino & $2(18 \cdot 2 \%)$ & $11(9 \cdot 9 \%)$ & $14(12 \cdot 4 \%)$ & $27(11.5 \%)$ \\
\hline Not Hispanic-Latino & $9(81 \cdot 8 \%)$ & $98(88.3 \%)$ & $99(87.6 \%)$ & $206(87 \cdot 7 \%)$ \\
\hline Other & 0 & $2(1 \cdot 8 \%)$ & 0 & $2(0.9 \%)$ \\
\hline \multicolumn{5}{|l|}{ HCV genotype } \\
\hline $1 \mathrm{a}$ & $10(90 \cdot 9 \%)$ & $53(47 \cdot 7 \%)$ & $59(52 \cdot 2 \%)$ & $122(51 \cdot 9 \%)$ \\
\hline $1 b$ & $1(9 \cdot 1 \%)$ & $58(52 \cdot 3 \%)$ & $53(46 \cdot 9 \%)$ & $112(47 \cdot 7 \%)$ \\
\hline 1 other & 0 & 0 & $1(0.9 \%)$ & $1(0.4 \%)$ \\
\hline \multicolumn{5}{|l|}{ IL28B } \\
\hline CC & $2(18 \cdot 2 \%)$ & $30(27 \cdot 0 \%)$ & $30(26 \cdot 5 \%)$ & $62(26 \cdot 4 \%)$ \\
\hline Non-CC & $9(81 \cdot 8 \%)$ & $79(71 \cdot 2 \%)$ & $83(73 \cdot 5 \%)$ & $171(72 \cdot 8 \%)$ \\
\hline Missing & 0 & $2(1 \cdot 8 \%)$ & 0 & $2(0.9 \%)$ \\
\hline \multicolumn{5}{|l|}{ Cirrhosis } \\
\hline No & $11(100 \cdot 0 \%)$ & $104(93 \cdot 7 \%)$ & $106(93 \cdot 8 \%)$ & $221(94 \cdot 0 \%)$ \\
\hline Yes & 0 & $7(6 \cdot 2 \%)$ & $7(6 \cdot 2 \%)$ & $14(6.0 \%)$ \\
\hline \multicolumn{5}{|l|}{ Hepatitis fibrosis stage } \\
\hline F0-F2 & $11(100 \%)$ & $76(68.5 \%)$ & $76(67 \cdot 3 \%)$ & $163(69 \cdot 4 \%)$ \\
\hline F3 & 0 & $13(11 \cdot 7 \%)$ & $15(13 \cdot 3 \%)$ & $28(11.9 \%)$ \\
\hline $\mathrm{F} 4$ & 0 & $7(6 \cdot 3 \%)$ & $7(6 \cdot 2 \%)$ & $14(6 \cdot 0 \%)$ \\
\hline Other* & 0 & $15(13 \cdot 5 \%)$ & $15(13 \cdot 3 \%)$ & $30(12 \cdot 8 \%)$ \\
\hline \multicolumn{5}{|l|}{ Baseline HCV RNA } \\
\hline$\leq 800000 \mathrm{IU} / \mathrm{mL}$ & $3(27 \cdot 3 \%)$ & $50(45.0 \%)$ & $47(41 \cdot 6 \%)$ & $100(42 \cdot 6 \%)$ \\
\hline$>800000 \mathrm{IU} / \mathrm{mL}$ & $8(72 \cdot 7 \%)$ & $61(55 \cdot 0 \%)$ & $66(58.4 \%)$ & $135(57 \cdot 4 \%)$ \\
\hline \multicolumn{5}{|l|}{ HCV treatment history } \\
\hline Naive & $10(90 \cdot 9 \%)$ & $91(82 \cdot 0 \%)$ & $88(77 \cdot 9 \%)$ & $189(80 \cdot 4 \%)$ \\
\hline Experienced & $1(9 \cdot 1 \%)$ & $20(18 \cdot 0 \%)$ & $25(22 \cdot 1 \%)$ & $46(19 \cdot 6 \%)$ \\
\hline \multicolumn{5}{|l|}{ Dialysis status } \\
\hline On dialysis & $6(54 \cdot 5 \%)$ & $86(77.5 \%)$ & $87(77 \cdot 0 \%)$ & $179(76 \cdot 2 \%)$ \\
\hline Not on dialysis & $5(45 \cdot 5 \%)$ & $25(22.5 \%)$ & $26(23.0 \%)$ & $56(23.8 \%)$ \\
\hline \multicolumn{5}{|l|}{ Diabetes status } \\
\hline Diabetes & $6(54 \cdot 5 \%)$ & $38(34 \cdot 2 \%)$ & $36(31 \cdot 9 \%)$ & $80(34 \cdot 0 \%)$ \\
\hline No diabetes & $5(45 \cdot 5 \%)$ & $73(65 \cdot 8 \%)$ & $77(68.1 \%)$ & $155(66 \cdot 0 \%)$ \\
\hline \multicolumn{5}{|c|}{ Chronic kidney disease stage } \\
\hline 4 & $4(36 \cdot 4 \%)$ & $18(16 \cdot 2 \%)$ & $22(19 \cdot 5 \%)$ & $44(18 \cdot 7 \%)$ \\
\hline \multirow[t]{2}{*}{5} & $7(63 \cdot 6 \%)$ & $93(83.8 \%)$ & $91(80 \cdot 5 \%)$ & $191(81 \cdot 3 \%)$ \\
\hline & & & \multicolumn{2}{|c|}{ (Table 1 continues on next page) } \\
\hline
\end{tabular}

An external data monitoring committee met when $50 \%$ of patients had completed treatment week 4 or discontinued before treatment week 4 and again when all patients had completed treatment week 8 or discontinued before treatment week 8. After each meeting, it was recommended the study continue as planned.

\section{Outcomes}

The primary efficacy outcome was a non-randomised comparison of sustained virological response at 12 weeks after the end of therapy (SVR12) for patients in the immediate treatment group and intensive pharmacokinetic population versus historical control patients with a reference SVR12 of 45\% (appendix). Relapse was defined as detectable HCV RNA following the end of therapy, after undetectable at end of treatment. The primary safety outcome was a comparison between the randomised immediate treatment and deferred treatment groups. Tier 1 safety events were defined as alanine aminotransferase (ALT) or aspartate aminotransferase (AST) greater than $500 \mathrm{IU} / \mathrm{L}$; ALT or AST greater than three times the baseline and greater than $100 \mathrm{IU} / \mathrm{L}$; alkaline phosphatase greater than three times the upper limit of normal. Tier 2 safety events were defined as patients with one or more adverse events, a drug-related adverse event, a serious adverse event, a serious renal adverse event, a serious and drug-related adverse event, an adverse event leading to discontinuation from treatment, and changes in renal function (increasing dialysis frequency in patients on haemodialysis at baseline, initiation of maintenance haemodialysis in patients not on haemodialysis at baseline, or an increase in chronic kidney disease stage). Tier 2 safety parameters also included change from baseline in serum creatinine, blood urea nitrogen, and eGFR in patients not receiving haemodialysis at baseline. Pharmacokinetic data are not reported here. Secondary endpoints not reported here are the SVR24 for the immediate treatment group and SVR12 for the active treatment phase of the deferred group.

\section{Statistical analysis}

According to the primary hypothesis, patients receiving grazoprevir and elbasvir in the immediate treatment group and intensive pharmacokinetic population will achieve an SVR12 rate higher than the reference rate of $45 \%$ (appendix). This value is based on a meta-analysis indicating an SVR rate of 39\% in patients with stages 3-5 chronic kidney disease receiving interferon monotherapy ${ }^{20}$ and an SVR of $40 \%$ in patients with HCV genotype 1 infection without renal disease receiving peginterferon and ribavirin. ${ }^{21}$ The primary hypothesis was tested at a two-sided significance level (type I error) of 0.05 using an exact test for a binomial proportion. A 95\% CI was also constructed for the SVR12 rate using the Clopper-Pearson method on non-randomised populations.

The modified full analysis set served as the primary population for the analysis of efficacy, and included patients assigned to the immediate treatment group or assigned to the intensive pharmacokinetic group, excluding those who failed to receive one or more doses 
of drug, died, or discontinued from the study early for reasons unrelated to hepatitis $\mathrm{C}$ treatment. A secondary analysis including all patients who received at least one dose of study drug (full analysis set) was also done.

Target enrolment was 105 patients in each of the immediate treatment group and deferred treatment group, and 10 patients in the intensive pharmacokinetic cohort. With this sample size, there is $95 \%$ or more power to show that the SVR12 rate in patients receiving grazoprevir and elbasvir is higher than the reference SVR12 rate of $45 \%$, at an overall one-sided $0.025 \alpha$ level, if the true SVR12 rate of grazoprevir and elbasvir is about $65 \%$. A post-hoc descriptive summary of SVR4 (at week 16) in patients receiving placebo in the deferred treatment group is also reported. SVR12 cannot be reported for the deferred treatment group because these patients began active treatment at week 16 .

The full analysis set population was used for the analysis of safety data. Tier 1 event rates were compared between immediate treatment and deferred treatment groups: p values and 95\% CIs were calculated using the stratified Miettinen and Nurminen method with baseline dialysis status as the strata. ${ }^{22}$ Safety events occurring up to 14 days after completion of treatment were captured to ensure the reporting of events that might be related to persistence of study drug. The study is registered at ClinicalTrials.gov, number NCT02092350.

\section{Role of the funding source}

Merck Sharp \& Dohme Corp contributed to trial management, data collection, statistical analyses, writing, and review of the report. All authors had access to the data, reviewed and approved the final report, and take full responsibility for the veracity of the data and statistical analysis. The corresponding author had full access to the data and had final responsibility for the decision to submit for publication.

\section{Results}

In total, 237 patients were enrolled and 235 received one or more doses of study drug between March 30, 2014, and Nov 28, 2014. Of these, 224 were assigned to the immediate treatment group $(\mathrm{n}=111)$ or deferred treatment group $(\mathrm{n}=113$ ), and an additional 11 patients were assigned to the intensive pharmacokinetic treatment group (figure 1).

Demographic and baseline characteristics were generally balanced between the immediate treatment group, intensive pharmacokinetic, and deferred treatment group populations (table 1). Overall, 179 (76\%) of 235 patients were on haemodialysis and 191 (81\%) had chronic kidney disease stage 5 at baseline. 80 (34\%) patients had diabetes, 96 (41\%) had cardiovascular disease, 122 (52\%) had HCV genotype 1a infection, 189 (80\%) were HCV treatment-naive, and 14 (6\%) were cirrhotic.

of the 122 patients in the immediate treatment and intensive pharmacokinetic population, six were excluded from the modified full analysis set population for reasons other than virological failure (death, lost to follow-up, noncompliance, patient withdrawal, and withdrawal by physician due to violent behaviour; figure 1). All six patients had HCV RNA less than $15 \mathrm{IU} / \mathrm{mL}$ at time of

\begin{tabular}{|c|c|c|c|c|}
\hline & $\begin{array}{l}\text { Grazoprevir and } \\
\text { elbasvir } \\
\text { pharmacokinetic } \\
\text { population }(n=11)\end{array}$ & $\begin{array}{l}\text { Grazoprevir and } \\
\text { elbasvir immediate } \\
\text { treatment group } \\
(n=111)\end{array}$ & $\begin{array}{l}\text { Grazoprevir and } \\
\text { elbasvir deferred } \\
\text { treatment group } \\
(n=113)\end{array}$ & Total $(n=235)$ \\
\hline \multicolumn{5}{|c|}{ (Continued from previous page) } \\
\hline \multicolumn{5}{|c|}{ Previous renal transplant } \\
\hline Yes & $2(18 \cdot 2 \%)$ & $15(13 \cdot 5 \%)$ & $28(24 \cdot 8 \%)$ & $45(19 \cdot 1 \%)$ \\
\hline No & $9(81 \cdot 8 \%)$ & $96(86 \cdot 5 \%)$ & $85(75 \cdot 2 \%)$ & $190(80 \cdot 9 \%)$ \\
\hline \multicolumn{5}{|c|}{ Primary aetiology of renal disease } \\
\hline Hypertension & $4(36 \cdot 4 \%)$ & $46(41 \cdot 4 \%)$ & $42(37 \cdot 2 \%)$ & $92(39 \cdot 1 \%)$ \\
\hline Type 1 diabetes & $2(18 \cdot 2 \%)$ & $4(3 \cdot 6 \%)$ & $7(6 \cdot 2 \%)$ & $13(5 \cdot 5 \%)$ \\
\hline Type 2 diabetes & $2(18 \cdot 2 \%)$ & $19(17 \cdot 1 \%)$ & $25(22 \cdot 1 \%)$ & $46(19 \cdot 6 \%)$ \\
\hline $\begin{array}{l}\text { Congenital cystic } \\
\text { kidney disease }\end{array}$ & 0 & $4(3 \cdot 6 \%)$ & $1(0 \cdot 9 \%)$ & $5(2 \cdot 1 \%)$ \\
\hline $\begin{array}{l}\text { Chronic } \\
\text { autoimmune } \\
\text { glomerulonephritis }\end{array}$ & 0 & $11(9 \cdot 9 \%)$ & $5(4 \cdot 4 \%)$ & $16(6 \cdot 8 \%)$ \\
\hline Pyelonephritis & 0 & $2(1 \cdot 8 \%)$ & 0 & $2(0.9 \%)$ \\
\hline $\begin{array}{l}\text { Urinary tract } \\
\text { obstruction }\end{array}$ & 0 & $4(3 \cdot 6 \%)$ & $2(1 \cdot 8 \%)$ & $6(2 \cdot 6 \%)$ \\
\hline Cryoglobulinaemia & $2(18 \cdot 2 \%)$ & $2(1 \cdot 8 \%)$ & 0 & $4(1 \cdot 7 \%)$ \\
\hline Other & $1(9 \cdot 1 \%)$ & $19(17 \cdot 1 \%)$ & $31(27 \cdot 4 \%)$ & $51(21 \cdot 7 \%)$ \\
\hline
\end{tabular}

Data are $\mathrm{n}(\%)$ or mean (SD). IL28B=interleukin 28B gene. ${ }^{*}$ Other category applies to 30 patients assessed by Fibrotest but could not be considered cirrhotic.

Table 1: Patient demographics

\begin{tabular}{|c|c|c|}
\hline & $\begin{array}{l}\text { Grazoprevir and elbasvir } \\
\text { immediate treatment group and } \\
\text { pharmacokinetic population }\end{array}$ & $\begin{array}{l}\text { Grazoprevir and elbasvir } \\
\text { deferred treatment group }\end{array}$ \\
\hline \multicolumn{3}{|l|}{ SVR12 (HCV RNA<LLoQ) } \\
\hline Modified full analysis set & $115 / 116(99 \cdot 1 \%[95 \cdot 3-100 \cdot 0])$ & .. \\
\hline Full analysis set & $115 / 122(94 \cdot 3 \%[88 \cdot 5-97 \cdot 7])$ & .. \\
\hline \multicolumn{3}{|c|}{ On-treatment and follow-up virological response (mFAS, TND)* } \\
\hline Treatment week 2 & $51 / 122(41 \cdot 8 \%)$ & $0 / 113$ \\
\hline Treatment week 4 & $94 / 121(77 \cdot 7 \%)$ & $1 / 113(0 \cdot 9 \%)$ \\
\hline Treatment week 12 & $119 / 119(100 \%)$ & $1 / 113(0 \cdot 9 \%)$ \\
\hline Follow-up week 4 & $117 / 118(99 \cdot 2 \%)$ & $1 / 113(0 \cdot 9 \%)$ \\
\hline \multicolumn{3}{|c|}{ On-treatment virological response $(\mathrm{mFAS}<\mathrm{LLoQ})^{*}$} \\
\hline Treatment week 2 & $81 / 122(66 \cdot 4 \%)$ & $0 / 113$ \\
\hline Treatment week 4 & $109 / 121(90 \cdot 1 \%)$ & $2 / 113+(1 \cdot 8 \%)$ \\
\hline Treatment week 12 & $119 / 119(100 \%)$ & $1 / 113(0 \cdot 9 \%)$ \\
\hline Follow-up week 4 & $118 / 118(100 \%)$ & $1 / 113(0 \cdot 9 \%)$ \\
\hline Relapse (mFAS) & $1 / 116(0 \cdot 9 \%)$ & .. \\
\hline \multicolumn{3}{|c|}{$\begin{array}{l}\text { Data are } \mathrm{n} / \mathrm{N}(\mathrm{SVR} \%[95 \% \mathrm{Cl}]) \text { or } \mathrm{n} / \mathrm{N}(\mathrm{SVR} \%) \text {. The } 95 \% \mathrm{Cl} \text { was estimated based on the Clopper-Pearson method. } \\
\text { SVR=sustained virological response. } \mathrm{HCV}=\text { hepatitis } \mathrm{C} \text { virus. LLoQ=lower limit of quantification (HCV RNA is detected } \\
\text { but }<15 \mathrm{IU} / \mathrm{mL} \text { ). mFAS=modified full analysis set. TND=HCV RNA target not detected (no calculated HCV RNA result } \\
\text { obtained (ie, HCV RNA undetectable). *Modified full analysis set was not defined for the deferred treatment group so } \\
\text { data are presented for the full analysis set population (all patients who received one or more doses of study drug). } \\
\text { tIn the deferred treatment group, two patients had HCV RNA }<\text { LLoQ at treatment week 4; one patient had } \\
\text { undetectable HCV RNA and one patient had detectable but unquantifiable HCV RNA. }\end{array}$} \\
\hline
\end{tabular}




\begin{tabular}{|c|c|c|c|}
\hline & $\mathrm{n} / \mathrm{m}$ & SVR12 $\left(\%\left[95 \% \mathrm{Cl}^{*}\right]\right)$ & \\
\hline All & $115 / 116$ & $99 \cdot 1(95 \cdot 3-100 \cdot 0)$ & \\
\hline \multicolumn{4}{|l|}{ Sex } \\
\hline Male & $88 / 89$ & $98.9(93.9-100 \cdot 0)$ & \\
\hline Female & $27 / 27$ & $100 \cdot 0(87 \cdot 2-100 \cdot 0)$ & \\
\hline \multicolumn{4}{|l|}{ Age, years } \\
\hline$<65$ & $95 / 96$ & $99 \cdot 0(94 \cdot 3-100 \cdot 0)$ & \\
\hline$\geq 65$ & 20/20 & $100 \cdot 0(83 \cdot 2-100 \cdot 0)$ & \\
\hline \multicolumn{4}{|l|}{ Race } \\
\hline White & $58 / 59$ & $98 \cdot 3(90 \cdot 9-100 \cdot 0)$ & \\
\hline African-American & $51 / 51$ & $100.0(93.0-100.0)$ & \\
\hline Asian & $5 / 5$ & $100 \cdot 0(47 \cdot 8-100 \cdot 0)$ & 4 \\
\hline \multicolumn{4}{|l|}{ HCV genotype } \\
\hline $1 \mathrm{a}$ & $61 / 61$ & $100 \cdot 0(94 \cdot 1-100 \cdot 0)$ & \\
\hline $1 b$ & $54 / 55$ & $98 \cdot 2(90 \cdot 3-100 \cdot 0)$ & \\
\hline \multicolumn{4}{|l|}{ IL28B genotype $\dagger$} \\
\hline CC & $28 / 28$ & $100 \cdot 0(87 \cdot 7-100 \cdot 0)$ & \\
\hline Non-CC & $86 / 87$ & $98.9(93 \cdot 8-100 \cdot 0)$ & \\
\hline \multicolumn{4}{|l|}{ Cirrhosis } \\
\hline No & $109 / 110$ & $99 \cdot 1(95 \cdot 0-100 \cdot 0)$ & \\
\hline Yes & $6 / 6$ & $100 \cdot 0(54 \cdot 1-100 \cdot 0)$ & 4 \\
\hline \multicolumn{4}{|l|}{ Baseline viral load } \\
\hline$\leq 800000 \mathrm{IU} / \mathrm{mL}$ & $50 / 50$ & $100 \cdot 0(92 \cdot 9-100 \cdot 0)$ & \\
\hline$>800000 \mathrm{IU} / \mathrm{mL}$ & $65 / 66$ & $98 \cdot 5(91 \cdot 8-100 \cdot 0)$ & \\
\hline \multicolumn{4}{|c|}{ Previous HCV treatment status } \\
\hline Naive & $96 / 96$ & $100 \cdot 0(96 \cdot 2-100 \cdot 0)$ & \\
\hline Experienced & $19 / 20$ & $95 \cdot 0(75 \cdot 1-99 \cdot 9)$ & 4 \\
\hline \multicolumn{4}{|l|}{ Dialysis } \\
\hline Yes & $86 / 87$ & $98.9(93.8-100 \cdot 0)$ & \\
\hline No & $29 / 29$ & $100.0(88.1-100.0)$ & \\
\hline \multicolumn{4}{|l|}{ Diabetes } \\
\hline Yes & $40 / 41$ & $97.6(87.1-99 \cdot 9)$ & \\
\hline No & $75 / 75$ & $100.0(95 \cdot 2-100 \cdot 0)$ & \\
\hline \multicolumn{4}{|c|}{ Chronic kidney disease stage } \\
\hline Stage 4 & $22 / 22$ & $100 \cdot 0(84 \cdot 6-100 \cdot 0)$ & \\
\hline \multirow[t]{3}{*}{ Stage 5} & 93/94 & $98 \cdot 9(94 \cdot 2-100 \cdot 0)$ & \\
\hline & & & 90 \\
\hline & & & SVR12 (\%[95\% CI]) \\
\hline
\end{tabular}

Figure 2: SVR12 subgroup analyses (modified full analysis set)

$S V R=$ sustained virological response. $m=$ number of patients included in the analysis. $n=$ number of patients who achieved SVR12 (HCV RNA < LLoQ [ $<15 \mathrm{IU} / \mathrm{mL}])$ at 12 weeks after end of treatment. *Based on the Clopper-Pearson method. †One patient was missing baseline IL28B genotype.

discontinuation. Of the 116 remaining patients (immediate treatment group, $\mathrm{n}=105$; intensive pharmacokinetic group, $\mathrm{n}=11), 115$ (99\%) achieved SVR12, a rate better than the historical control rate of $45 \%(\mathrm{p}<0 \cdot 001)$. One noncirrhotic patient with HCV genotype $1 \mathrm{~b}$ infection and chronic kidney disease stage 5 relapsed 12 weeks after the end of treatment (table 2).

In the full analysis set population, 115 (94\%) of 122 patients achieved SVR12. Of the seven patients who did not achieve SVR12, six patients discontinued the study for reasons other than virological failure and one patient relapsed.

High response rates were observed in all subgroups (figure 2), including haemodialysis and non-haemodialysis, and those with characteristics historically associated with poor response to HCV therapy. In particular, SVR12 was achieved in $51(100 \%)$ of 51 African American patients $86(99 \%)$ of 87 patients with the IL28B non-CC genotype, $40(98 \%)$ of 41 patients with diabetes, and all six patients with cirrhosis.

The SVR4 rate in patients receiving placebo in the deferred treatment group was one $(<1 \%)$ of 113 . HCV RNA was undetectable in one patient receiving placebo 4 weeks after the end of the placebo treatment period. This patient denied taking any HCV therapy outside the study, had not initiated deferred active therapy, and it was confirmed that the study drug dispensed during the treatment period was placebo.

Baseline NS3/4A or NS5A resistance-associated variants were detected in $36(32 \cdot 1 \%)$ of 112 and $17(14 \cdot 8 \%)$ of 115 patients in the immediate treatment group and intensive pharmacokinetic population with sequencing data, respectively (based on population sequencing). SVR12 was achieved in $36(100 \%)$ of 36 and 16 (94.1\%) of 17 of these patients, respectively. The patient who relapsed had an NS5A L31M mutation at baseline.

The frequencies of adverse events were comparable between the immediate treatment and deferred treatment groups ( $76 \%$ vs $84 \%$; table 3 ), and most adverse events were of mild or moderate intensity in both treatment groups. The most common adverse events ( $\geq 10 \%$ frequency) were headache, nausea, and fatigue and were comparable in the two groups. Cardiac serious adverse events were reported in two patients in the immediate treatment group (one cardiac arrest, one myocardial infarction) and three in the deferred treatment group (two myocardial infarctions, one cardiomyopathy; appendix). Two cases of congestive heart failure occurred in the immediate treatment group within 14 days of the end of treatment; one of these, judged by the investigator to be drug-related, was reported 6 weeks after study treatment ended. A total of $16(14 \%)$ patients in the immediate treatment group and $19(17 \%)$ patients in the deferred treatment group reported a serious adverse event during treatment or within 14 days after the end of treatment (appendix). The serious adverse events reported were consistent with the underlying comorbidities and complications within this patient population. The only serious adverse events reported in more than one patient in the immediate treatment group were hypertension and pneumonia $(n=2$ each). There were no serious adverse events considered to be drug-related in the immediate treatment group.

In the deferred treatment group, serious adverse events reported in more than one patient were upper gastrointestinal haemorrhage $(n=2)$, myocardial infarction $(\mathrm{n}=2)$, and aortic aneurysm $(\mathrm{n}=2)$. Increased lipase was the only serious drug-related adverse event in the deferred treatment group.

There were no discontinuations due to an adverse event in the immediate treatment group versus five patients in the deferred treatment group (one each 
of abdominal pain, elevated ALT and AST, atrial fibrillation with myocardial infarction, increased lipase, and acute myocardial infarction). There were four deaths, none considered related to study drug, during the initial treatment plus 14 day period. One $(1 \%)$ patient in the immediate treatment group died from cardiac arrest and three $(3 \%)$ in the deferred treatment group died from aortic aneurysm, pneumonia, and unknown cause of death.

The frequencies and severities of liver function measures were comparable between the immediate treatment and deferred treatment groups (table 3). Rises in ALT and AST were more common among patients receiving placebo than grazoprevir and elbasvir. Rises in bilirubin and alkaline phosphatase and change in blood urea nitrogen from baseline were comparable in both treatment groups (appendix). A higher frequency of low haemoglobin $(8 \cdot 5-<10 \cdot 0 \mathrm{~g} / \mathrm{dL})$ was noted in the immediate treatment group $(n=27,24 \cdot 3 \%)$ than in the deferred treatment group $(n=19,16 \cdot 8 \%)$. Erythropoietin stimulating agents were used during the treatment period by 27 (24\%) patients in the immediate treatment group and $34(30 \%)$ patients in the deferred treatment group. No adverse events suggestive of liver decompensation were reported.

The frequencies of renal system adverse events were generally comparable between treatment groups (appendix). Two patients in the immediate treatment group initiated maintenance dialysis during the study and six patients (immediate treatment group, $n=4$; deferred treatment group, $\mathrm{n}=2$ ) not on dialysis at baseline had a change in chronic kidney disease stage, based on a decrease in eGFR from $15-29 \mathrm{~mL} / \mathrm{min}$ per $1.73 \mathrm{~m}^{2}$ at baseline to less than $15 \mathrm{~mL} / \mathrm{min}$ per $1.73 \mathrm{~m}^{2}$. Worsening of proteinuria was reported in four patients in the immediate treatment group (dialysis, $n=1$; no dialysis, $\mathrm{n}=3$ ) and eight patients in the deferred treatment group (dialysis, $n=4$; no dialysis, $n=4$ ). There was no consistent change in mean eGFR or creatinine in either treatment group (appendix).

\section{Discussion}

This study shows that the combination of grazoprevir and elbasvir for 12 weeks is an effective treatment regimen for patients with $\mathrm{HCV}$ genotype 1 infection and advanced stage 4-5 chronic kidney disease, including patients on haemodialysis and those considered difficult to treat with interferon-based antiviral therapy. Only one $(<1 \%)$ of 116 patients who completed treatment with grazoprevir and elbasvir did not achieve SVR12. This non-cirrhotic patient with a NS5A L31M mutation at baseline relapsed after having undetectable HCV RNA at the end of treatment and at the 4-week post-treatment visit. No patient had on-treatment virological breakthrough. The short, 12 -week duration of treatment with grazoprevir and elbasvir might allow waitlisting of patients for kidney transplant while on treatment for

\begin{tabular}{|c|c|c|}
\hline & $\begin{array}{l}\text { Grazoprevir and elbasvir } \\
\text { immediate treatment group } \\
(n=111)\end{array}$ & $\begin{array}{l}\text { Grazoprevir and elbasvir } \\
\text { deferred treatment group } \\
(n=113)\end{array}$ \\
\hline Any adverse event ${ }^{*} \dagger$ & $84(75 \cdot 7 \%)$ & $95(84 \cdot 1 \%)$ \\
\hline Headache & $19(17 \cdot 1 \%)$ & $19(16 \cdot 8 \%)$ \\
\hline Nausea & $17(15 \cdot 3 \%)$ & $18(15 \cdot 9 \%)$ \\
\hline Fatigue & $11(9 \cdot 9 \%)$ & $17(15 \cdot 0 \%)$ \\
\hline Insomnia & $7(6 \cdot 3 \%)$ & $12(10 \cdot 6 \%)$ \\
\hline Dizziness & $6(5 \cdot 4 \%)$ & $18(15 \cdot 9 \%)$ \\
\hline Diarrhoea & $6(5 \cdot 4 \%)$ & $15(13 \cdot 3 \%)$ \\
\hline Drug-related adverse event $\dagger$ & $38(34 \cdot 2 \%)$ & $39(34 \cdot 5 \%)$ \\
\hline Serious adverse event $†$ & $16(14 \cdot 4 \%)$ & $19(16 \cdot 8 \%)$ \\
\hline Drug-related serious adverse event $\dagger$ & 0 & $1(0 \cdot 9 \%)$ \\
\hline Discontinuation due to an adverse event & 0 & $5 \ddagger(4 \cdot 4 \%)$ \\
\hline Deaths & $1(0 \cdot 8 \%)$ & $3(2 \cdot 7 \%)$ \\
\hline \multicolumn{3}{|l|}{ Lowest haemoglobin on treatment§ } \\
\hline $8 \cdot 5-10.0 \mathrm{~g} / \mathrm{dL}$ & $27(24 \cdot 3 \%)$ & $19(16 \cdot 8 \%)$ \\
\hline$<8.5 \mathrm{~g} / \mathrm{dL}$ & $5(4 \cdot 5 \%)$ & $5(4 \cdot 4 \%)$ \\
\hline \multicolumn{3}{|l|}{ Alanine aminotransferase $§$} \\
\hline $1.1-2.5 \times$ baseline & $2(1.8 \%)$ & $36(31 \cdot 9 \%)$ \\
\hline$>2.5 \times$ baseline & $1(0 \cdot 8 \%)$ & $6(5 \cdot 3 \%)$ \\
\hline$>5.0 \times$ baseline & 0 & $1(0.9 \%)$ \\
\hline \multicolumn{3}{|l|}{ Aspartate aminotransferase $\$$} \\
\hline $1.1-2.5 \times$ baseline & $4(3 \cdot 6 \%)$ & $38(33 \cdot 6 \%)$ \\
\hline$>2.5 \times$ baseline & 0 & $4(4 \cdot 6 \%)$ \\
\hline$>5.0 \times$ baseline & 0 & 0 \\
\hline \multicolumn{3}{|l|}{ Bilirubin $\S$} \\
\hline$>2.5-5.0 \times$ baseline & $1(0 \cdot 9 \%)$ & $3(2 \cdot 7 \%)$ \\
\hline$>5 \cdot 0-10 \cdot 0 \times$ baseline & 0 & 0 \\
\hline$>10.0 \times$ baseline & 0 & 0 \\
\hline \multicolumn{3}{|l|}{ Alkaline phosphatase $§$} \\
\hline $1.1-2.5 \times$ baseline & $42(37 \cdot 8 \%)$ & $36(31 \cdot 9 \%)$ \\
\hline$>2.5 \times$ baseline & 0 & 0 \\
\hline$>5.0 \times$ baseline & 0 & 0 \\
\hline Creatinine $\S>2.5 \times$ baseline & $1(1 \cdot 2 \%)$ & 0 \\
\hline $\begin{array}{l}\text { Change in blood urea nitrogen }(\mathrm{mg} / \mathrm{L}) \text { from } \\
\text { baseline at treatment week } 12 \S \uparrow\end{array}$ & $-1 \cdot 5(3 \cdot 6)$ & $0.9(2.6)$ \\
\hline \multicolumn{3}{|c|}{$\begin{array}{l}\text { Data are } n(\%) \text { or mean (SE). *Incidence } 10 \% \text { or more in one or more treatment groups during the initial treatment period } \\
\text { and for } 14 \text { days after the completion of treatment (all patients as treated). }+ \text { Number of patients with the specific adverse } \\
\text { event. łAbdominal pain, elevated alanine transaminase and aspartate transaminase, acute myocardial infarction, atrial } \\
\text { fibrillation with myocardial infarction, and increased lipase. SData presented for patients with more than } 1.0 \text { change from } \\
\text { baseline. } \text { IPatients not on dialysis at baseline (immediate treatment group, } n=25 \text {; deferred treatment group, } n=24 \text { ). }\end{array}$} \\
\hline
\end{tabular}

HCV infection, a practice that was previously difficult due to the 24-48 weeks of treatment needed with peginterferon and ribavirin regimens.

The deferred treatment group was used to provide a comparator for safety data collected in the immediate treatment group, given the substantial comorbidities seen in patients with stage 4-5 chronic kidney disease. The safety profiles of patients who received grazoprevir and elbasvir and placebo treatment were comparable, with similar frequencies of adverse events, serious adverse events, and renal and hepatic laboratory 
abnormalities. No patient discontinued due to an adverse event in the immediate treatment group. Previous studies of first-generation HCV protease inhibitors have shown a reversible decline in eGFR during treatment; ${ }^{23}$ however, no such changes were noted in the present study, and no differences in renal function were noted between treatment groups. The five patients with cardiac serious adverse events reflect the known high prevalence of hypertension, diabetes, and cardiovascular disease in patients with chronic kidney disease, especially those on haemodialysis.

SVR12 response rates in the present study are consistent with those reported in studies of patients with HCV genotype 1 infection and normal renal function. In the C-WORTHY study, a 12-week regimen of grazoprevir and elbasvir resulted in SVR12 in $98 \%$ of non-cirrhotic and $97 \%$ of cirrhotic patients. ${ }^{11,12}$ High response rates with grazoprevir and elbasvir have also recently been reported in patients with HCV infection and normal renal function with previous non-response to first-generation direct-acting antiviral agents and in treatment-naive patients. ${ }^{13,14}$ Efficacy in the present study was also generally comparable with that of a 12 -week regimen of sofosbuvir plus ledipasvir that achieved an SVR12 rate of 96-99\% in non-cirrhotic and 94\% in cirrhotic treatment-naive patients with HCV genotype 1 infection and without chronic kidney disease stage $4-5 .{ }^{24,25}$

There are limitations to the present study. Patient numbers in some subgroups were small. Only $14(6 \%)$ cirrhotic patients were included. Also, patients with decompensated liver disease and those receiving peritoneal dialysis were excluded. The results of the C-SURFER study therefore cannot be generalised to all patient subgroups. A recent study including 205 Taiwanese haemodialysis patients with genotype $1 \mathrm{~b}$ HCV infection reported an SVR rate of $64 \% .{ }^{26}$ These data suggest that an SVR rate higher than our historical control rate of $45 \%$ is achievable in Taiwanese patients with HCV genotype $1 \mathrm{~b}$ infection on haemodialysis and receiving peginterferon plus ribavirin for 48 weeks. Their result could be an overestimate of the treatment response since their study population was all Asian and thus a high percentage carried the IL28B CC genotype which is strongly predictive of SVR. Finally, the present study did not have an active comparator because of the restricted treatment options available for HCV infection in patients with advanced chronic kidney disease.

In conclusion, the results from the C-SURFER study suggest that a once-daily oral regimen of grazoprevir and elbasvir for 12 weeks has an acceptable safety profile and can achieve high rates of SVR in patients with HCV genotype 1 infection and advanced chronic kidney disease. The results of this study show that the efficacy and safety profile of this combination is consistent across many patient subgroups, including those receiving haemodialysis.

\section{Contributors}

WG, MR, JW, EB, and SW were responsible for study concept and design. BJ, DR, DRN, AB, AML, MS, HM, PM, SP, M-CL, TH, PJZ, and EZ were responsible for acquisition of data. All authors had responsibility for analysis and interpretation of data. WG, DR, B-YN, and EB did the initial drafting of the manuscript. All authors critically revised the manuscript for important intellectual content. SW did the statistical analysis. All authors did a final review and approved the manuscript.

\section{Declaration of interests}

DR has served on advisory boards for Bristol-Myers Squibb and Merck. DRN has received research support from Abbvie, Boehringer Ingelheim, Bristol-Myers Squibb, Gilead, GlaxoSmithKline, Janssen, and Merck. AB has served on advisory committees for Merck and Chemocentryx. AML has served on advisory boards for Gilead and Janssen. MS has served on speakers bureau or received grants from Bristol-Myers Squibb, MSD, Gilead, AbbVie, Janssen, and Boehringer. HM has served on speakers bureau and advisory boards for Merck. PM has served on advisory boards for Merck. SP has served as a speaker for GlaxoSmithKline, Bristol-Myers Squibb, Boehringer Ingelheim,

Janssen, Gilead, Roche, MSD, Sanofi, Novartis, Vertex, and Abbvie; has received grants from Bristol-Myers Squibb, Gilead, Roche, and MSD; and has served as a board member for GlaxoSmithKline, Bristol-Myers Squibb, Boehringer Ingelheim, Janssen, Gilead, Roche, MSD, Sanofi, Novartis, Vertex, and Abbvie. M-CL has served as a speaker for Janssen, MSD, Gilead, and Bristol-Myers Squibb, and as a consultant for Janssen and Bristol-Myers Squibb. TH has received research grants from Abbvie, Boehringer-Ingelheim, Bristol-Myers Squibb, Eisai, Gilead Sciences, Idenix, Ikaria, Janssen, La Jolla Pharmaceuticals, Merck, Mochida, NGM BioPharmaceuticals, Roche, Ocera, Sundise, Salix, Taigen, Takeda, Tobria, Vertex, and Vital Therapies; served as a speaker for Baxter, Bristol-Myers Squibb, Gilead, Janssen, and Salix; and served on advisory boards for Abbvie and Bristol-Myers Squibb. PJZ has received research grants from Merck, Abbvie, and Bristol-Myers Squibb; and served on advisory boards for Abbvie and Janssen. EZ has served on advisory boards for BMS, Abbvie, Merck, Janssen, and Gilead, and as a speaker for Bristol-Myers Squibb, Abbvie, Merck, Janssen, Roche, Novartis, and Gilead. SW, B-YN, MR, EB, JW, and WG are employees and shareholders at Merck.

\section{Acknowledgments}

We thank Anita Howe and Mary Motyl for hepatitis C virus resistance sequence analyses. Medical writing and editorial assistance was provided by Tim Ibbotson and Beth McMahon-Wise of ApotheCom. This assistance was funded by Merck Sharp \& Dohme Corp, a subsidiary of Merck \& Co., Inc, Kenilworth, NJ, USA.

\section{References}

1 Fabrizi F, Dixit V, Messa P. Impact of hepatitis C on survival in dialysis patients: a link with cardiovascular mortality? J Viral Hepat 2012; 19: 601-07.

2 Butt AA, Wang X, Fried LF. HCV infection and the incidence of CKD. Am J Kidney Dis 2011; 57: 396-402.

3 Tsui JI, Vittinghoff E, Shlipak MG, et al. Association of hepatitis C seropositivity with increased risk for developing end-stage renal disease. Arch Intern Med 2007; 167: 1271-76.

4 Noureddine LA, Usman SA, Yu Z, Moorthi RN, Moe SM. Hepatitis C increases the risk of progression of chronic kidney disease in patients with glomerulonephritis. Am J Nephrol 2010; 32: 311-16.

5 Pereira BJG, Wright TL, Schmid CH, Levey AS. The impact of pretransplantation hepatitis $\mathrm{C}$ infection on the outcome of renal transplantation. Transplantation 1995; 60: 799-805.

6 Legendre C, Garrigue V, Le BC, et al. Harmful long-term impact of hepatitis $\mathrm{C}$ virus infection in kidney transplant recipients. Transplantation 1998; 65: 667-70.

7 Fabrizi F, Martin P, Dixit V, et al. Post-transplant diabetes mellitus and HCV seropositive status after renal transplantation: meta-analysis of clinical studies. Am J Transplant 2005; 5: 2433-40.

8 Bruchfeld A, Wilczek H, Elinder CG. Hepatitis C infection, time in renal-replacement therapy, and outcome after kidney transplantation. Transplantation 2004; 78: 745-50.

9 Cruzado JM, Carrera M, Torras J, Grinyo JM. Hepatitis C virus infection and de novo glomerular lesions in renal allografts. Am J Transplant 2001; 1: 171-78. 
10 Summa V, Ludmerer SW, McCauley JA, et al. MK-5172, a selective inhibitor of hepatitis C virus NS3/4a protease with broad activity across genotypes and resistant variants. Antimicrob Agents Chemother 2012; 56: 4161-67.

11 Sulkowski M, Hezode C, Gerstoft J, et al. Efficacy and safety of 8 weeks versus 12 weeks of treatment with grazoprevir (MK-5172) and elbasvir (MK-8742) with or without ribavirin in patients with hepatitis $\mathrm{C}$ virus genotype 1 mono-infection and HIV/hepatitis $\mathrm{C}$ virus co-infection (C-WORTHY): a randomised, open-label phase 2 trial. Lancet 2015; 385: 1087-97.

12 Lawitz E, Gane E, Pearlman B, et al. Efficacy and safety of 12 weeks versus 18 weeks of treatment with grazoprevir (MK-5172) and elbasvir (MK-8742) with or without ribavirin for hepatitis C virus genotype 1 infection in previously untreated patients with cirrhosis and patients with previous null response with or without cirrhosis (C-WORTHY): a randomised, open-label phase 2 trial. Lancet 2015; 385: 1075-86.

13 Forns X, Gordon SC, Zuckerman E, et al. Grazoprevir/elbasvir plus ribavirin for chronic HCV genotype-1 infection after failure of combination therapy containing a direct-acting antiviral agent. J Hepatol 2015; published online April 17. DOI:10.1016/ j.jhep.2015.04.009.

14 Zeuzem S, Ghalib R, Reddy KR, et al. Grazoprevir-elbasvir combination therapy for treatment-naive cirrhotic and noncirrhotic patients with chronic HCV genotype 1, 4, or 6 infection: a randomized trial. Ann Intern Med 2015; 163: 1-13.

15 Yeh WW, Caro L, Guo Z, et al. Pharmacokinetics of co-administered HCV protease inhibitor MK-5172 and NS5A inhibitor MK-8742 in volunteers with end-stage renal disease on hemodialysis or severe renal impairment not on hemodialysis. Hepatology 2014; 60 (suppl 4): 1940

16 Levey AS, Coresh J, Greene T, et al. Using standardized serum creatinine values in the modification of diet in renal disease study equation for estimating glomerular filtration rate. Ann Intern Med 2006; 145: 247-54.
17 Foucher J, Chanteloup E, Vergniol J, et al. Diagnosis of cirrhosis by transient elastography (FibroScan): a prospective study. Gut 2006; 55: 403-08.

18 Castera L, Forns X, Alberti A. Non-invasive evaluation of liver fibrosis using transient elastography. J Hepatol 2008; 48: 835-47.

19 Boursier J, de Ledinghen, V, Zarski JP, et al. Comparison of eight diagnostic algorithms for liver fibrosis in hepatitis C: new algorithms are more precise and entirely noninvasive. Hepatology 2012; 55: 58-67.

20 Fabrizi F, Dixit V, Messa P, Martin P. Interferon monotherapy of chronic hepatitis $\mathrm{C}$ in dialysis patients: meta-analysis of clinical trials. J Viral Hepat 2008; 15: 79-88.

21 McHutchison JG, Lawitz EJ, Shiffman ML, et al. Peginterferon alfa-2b or alfa-2a with ribavirin for treatment of hepatitis C infection. N Engl J Med 2009; 361: 580-93.

22 Miettinen $\mathrm{O}$, Nurminen M. Comparative analysis of two rates. Stat Med 1985; 4: 213-26.

23 Mauss S, Hueppe D, Alshuth U. Renal impairment is frequent in chronic hepatitis C patients under triple therapy with telaprevir or boceprevir. Hepatology 2014; 59: 46-48.

24 Afdhal N, Zeuzem S, Kwo P, et al. Ledipasvir and sofosbuvir for untreated HCV genotype 1 infection. N Engl J Med 2014; 370: 1889-98.

25 Kowdley KV, Gordon SC, Reddy KR, et al. Ledipasvir and sofosbuvir for 8 or 12 weeks for chronic HCV without cirrhosis. N Engl J Med 2014; 370: 1879-88.

26 Liu CH, Huang CF, Liu CJ, et al. Pegylated interferon- $\alpha 2$ a with or without low-dose ribavirin for treatment-naive patients with hepatitis $C$ virus genotype 1 receiving hemodialysis: a randomized trial. Ann Intern Med 2013; 159: 729-38. 


\section{Supplementary appendix}

This appendix formed part of the original submission and has been peer reviewed. We post it as supplied by the authors.

Supplement to: Roth D, Nelson DR, Bruchfeld A, et al. Grazoprevir plus elbasvir in treatment-naive and treatment-experienced patients with hepatitis $C$ virus genotype 1 infection and stage 4-5 chronic kidney disease (the C-SURFER study): a combination phase 3 study. Lancet 2015; published online Oct 6. http://dx.doi. org/10.1016/S0140-6736(15)00349-9. 


\section{Supplementary Materials}

564 Sample Size Considerations

565 Several considerations led us to choose a reference SVR of $45 \%$ for this study:

566 (1) Interferon (IFN) mono-therapy is recommended for HCV-infected patients with CKD stages

$5673-5$ who are on or not yet on maintenance dialysis therapy. The meta-analyses, conducted by

568 Fabrizi et al, revealed a summary SVR24 of 39\% (CI 32-46\%). ${ }^{1}$

569 (2) Given the substantial variation in the G1 proportion of the studies (ranging from 0 to 1 ) in the

570 Fabrizi meta-analyses, a bayesian logistic regression model for SVR was used to account for the

571 variation of G1 proportions. Twenty studies with G1 proportion were identified from the Fabrizi

572 paper and included in the re-analysis. Non-informative priors were used for the bayesian

573 random-effect model containing a random intercept and a fixed effect of G1 proportion. The

574 model predicts that, if the studies had enrolled $100 \% \mathrm{G} 1$, the posterior probability/confidence

575 that the true overall population mean for SVR rate would have been at most $45 \%$ is about 0.90 .

576 (3) A SVR of approximately $40 \%$ was observed in a large study of peginterferon/ribavirin in

577 3,070 HCV G1-infected patients without renal disease conducted in the United States. ${ }^{23}$ The

578 SVR response of patients with CKD stage 4/5 is not expected to be higher than that of the

579 general HCV population without renal disease. 
Supplementary Figure 1. Study Design.

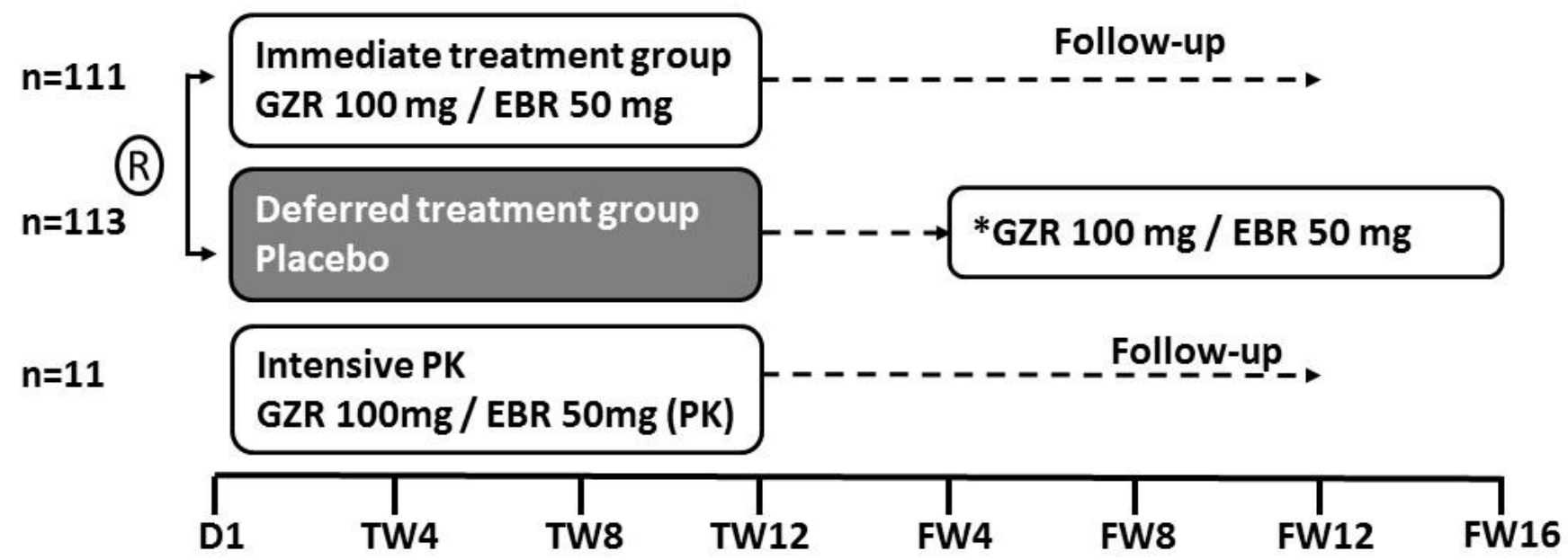

*Deferred open-label treatment arm (all randomised patients remained blinded to treatment until FW4).

GZR and EBR were administered as separate entities in the immediate and PK arms, and as a fixed dose-combination in the deferred arm. $\mathrm{R}=$ randomised 
Supplementary Table 1. Serious adverse events (initial treatment period and first 14 follow-up days; full analysis set)

\begin{tabular}{|c|c|c|c|c|c|c|c|c|}
\hline & \multicolumn{2}{|c|}{$\begin{array}{l}\text { Intensive PK arm: } \\
\text { GZR 100mg + EBR 50mg } \\
\text { for } 12 \text { Weeks }\end{array}$} & \multicolumn{2}{|c|}{$\begin{array}{l}\text { Immediate treatment arm: } \\
\text { GZR } 100 \mathrm{mg}+\text { EBR 50mg } \\
\text { for } 12 \text { Weeks }\end{array}$} & \multicolumn{2}{|c|}{$\begin{array}{l}\text { Deferred treatment arm: } \\
\text { GZR Placebo + EBR } \\
\text { Placebo for } 12 \text { Weeks }\end{array}$} & \multicolumn{2}{|c|}{$\begin{array}{c}\text { Immediate + Intensive PK } \\
\text { arms: GZR } 100 \mathrm{mg}+\mathrm{EBR} \\
50 \mathrm{mg} \text { for } 12 \mathrm{Weeks}\end{array}$} \\
\hline & $\mathrm{n}$ & $(\%)$ & $\mathrm{n}$ & $(\%)$ & $\mathrm{n}$ & $(\%)$ & $\mathrm{n}$ & $(\%)$ \\
\hline Subjects in population & 11 & & 111 & & 113 & & 122 & \\
\hline with one or more adverse events & 0 & $(0.0)$ & 16 & $(14.4)$ & 19 & $(16.8)$ & 16 & $(13.1)$ \\
\hline with no adverse events & 11 & $(100.0)$ & 95 & $(85.6)$ & 94 & $(83.2)$ & 106 & $(86.9)$ \\
\hline Cardiac disorders & $\mathbf{0}$ & $(\mathbf{0 . 0})$ & 2 & (1.8) & 3 & $(2.7)$ & 2 & (1.6) \\
\hline Acute myocardial infarction & 0 & $(0.0)$ & 0 & $(0.0)$ & 1 & $(0.9)$ & 0 & $(0.0)$ \\
\hline Angina unstable & 0 & $(0.0)$ & 0 & $(0.0)$ & 1 & $(0.9)$ & 0 & $(0.0)$ \\
\hline Atrial fibrillation & 0 & $(0.0)$ & 0 & $(0.0)$ & 1 & $(0.9)$ & 0 & $(0.0)$ \\
\hline Cardiac arrest & 0 & $(0.0)$ & 1 & $(0.9)$ & 0 & $(0.0)$ & 1 & $(0.8)$ \\
\hline Cardiomyopathy & 0 & $(0.0)$ & 0 & $(0.0)$ & 1 & $(0.9)$ & 0 & $(0.0)$ \\
\hline Myocardial infarction & 0 & $(0.0)$ & 1 & $(0.9)$ & 1 & $(0.9)$ & 1 & $(0.8)$ \\
\hline Gastrointestinal disorders & $\mathbf{0}$ & (0.0) & 2 & (1.8) & 4 & (3.5) & 2 & (1.6) \\
\hline Diarrhoea & 0 & $(0.0)$ & 1 & $(0.9)$ & 0 & $(0.0)$ & 1 & $(0.8)$ \\
\hline Gastritis & 0 & $(0.0)$ & 0 & $(0.0)$ & 1 & $(0.9)$ & 0 & $(0.0)$ \\
\hline $\begin{array}{l}\text { Localised intraabdominal fluid } \\
\text { collection }\end{array}$ & 0 & $(0.0)$ & 0 & $(0.0)$ & 1 & $(0.9)$ & 0 & $(0.0)$ \\
\hline Pancreatitis & 0 & $(0.0)$ & 1 & $(0.9)$ & 0 & $(0.0)$ & 1 & $(0.8)$ \\
\hline $\begin{array}{l}\text { Upper gastrointestinal } \\
\text { haemorrhage }\end{array}$ & 0 & $(0.0)$ & 0 & $(0.0)$ & 2 & $(1.8)$ & 0 & $(0.0)$ \\
\hline $\begin{array}{l}\text { General disorders and } \\
\text { administration site conditions }\end{array}$ & $\mathbf{0}$ & $(\mathbf{0 . 0})$ & $\mathbf{0}$ & $(\mathbf{0 . 0})$ & 1 & (0.9) & $\mathbf{0}$ & $(\mathbf{0 . 0})$ \\
\hline Death & 0 & $(0.0)$ & 0 & $(0.0)$ & 1 & $(0.9)$ & 0 & $(0.0)$ \\
\hline Infections and infestations & $\mathbf{0}$ & $(\mathbf{0 . 0})$ & 5 & $(4.5)$ & 3 & $(2.7)$ & 5 & (4.1) \\
\hline Abscess limb & 0 & $(0.0)$ & 1 & $(0.9)$ & 0 & $(0.0)$ & 1 & $(0.8)$ \\
\hline Appendicitis & 0 & $(0.0)$ & 1 & $(0.9)$ & 0 & $(0.0)$ & 1 & $(0.8)$ \\
\hline Citrobacter sepsis & 0 & $(0.0)$ & 1 & $(0.9)$ & 0 & $(0.0)$ & 1 & $(0.8)$ \\
\hline Enterobacter sepsis & 0 & $(0.0)$ & 1 & $(0.9)$ & 0 & $(0.0)$ & 1 & $(0.8)$ \\
\hline Haematoma infection & 0 & $(0.0)$ & 0 & $(0.0)$ & 1 & $(0.9)$ & 0 & $(0.0)$ \\
\hline Infected fistula & 0 & $(0.0)$ & 0 & $(0.0)$ & 1 & $(0.9)$ & 0 & $(0.0)$ \\
\hline Osteomyelitis & 0 & $(0.0)$ & 1 & $(0.9)$ & 0 & $(0.0)$ & 1 & $(0.8)$ \\
\hline Pneumonia & 0 & $(0.0$ & 2 & $(1.8)$ & 1 & $(0.9)$ & 2 & (1.6) \\
\hline
\end{tabular}




\begin{tabular}{|c|c|c|c|c|c|c|c|c|}
\hline $\begin{array}{l}\text { Injury, poisoning and } \\
\text { procedural complications }\end{array}$ & $\mathbf{0}$ & $(0.0)$ & 1 & $(0.9)$ & 2 & (1.8) & 1 & $(0.8)$ \\
\hline Arteriovenous fistula aneurysm & 0 & $(0.0)$ & 0 & $(0.0)$ & 1 & $(0.9)$ & 0 & $(0.0)$ \\
\hline Dialysis related complication & 0 & $(0.0)$ & 1 & $(0.9)$ & 0 & $(0.0)$ & 1 & $(0.8)$ \\
\hline Postoperative fever & 0 & $(0.0)$ & 0 & $(0.0)$ & 1 & $(0.9)$ & 0 & $(0.0)$ \\
\hline Procedural pain & 0 & $(0.0)$ & 1 & $(0.9)$ & 0 & $(0.0)$ & 1 & $(0.8)$ \\
\hline Investigations & $\mathbf{0}$ & $(0.0)$ & $\mathbf{0}$ & $(\mathbf{0 . 0})$ & 2 & (1.8) & $\mathbf{0}$ & $(0.0)$ \\
\hline $\begin{array}{l}\text { Blood alkaline phosphatase } \\
\text { increased }\end{array}$ & 0 & $(0.0)$ & 0 & $(0.0)$ & 1 & $(0.9)$ & 0 & $(0.0)$ \\
\hline Lipase increased & 0 & $(0.0)$ & 0 & $(0.0)$ & 1 & $(0.9)$ & 0 & $(0.0)$ \\
\hline $\begin{array}{l}\text { Metabolism and nutrition } \\
\text { disorder }\end{array}$ & $\mathbf{0}$ & $(\mathbf{0 . 0})$ & 2 & (1.8) & 3 & $(2.7)$ & 2 & (1.6) \\
\hline Dehydration & 0 & $(0.0)$ & 1 & $(0.9)$ & 0 & $(0.0)$ & 1 & $(0.8)$ \\
\hline Fluid overload & 0 & $(0.0)$ & 1 & $(0.9)$ & 1 & $(0.9)$ & 1 & $(0.8)$ \\
\hline Hyperglycaemia & 0 & $(0.0)$ & 0 & $(0.0)$ & 1 & $(0.9)$ & 0 & $(0.0)$ \\
\hline Hyperkalaemia & 0 & $(0.0)$ & 0 & $(0.0)$ & 1 & $(0.9)$ & 0 & $(0.0)$ \\
\hline $\begin{array}{l}\text { Musculoskeletal and } \\
\text { connective tissue disorders }\end{array}$ & $\mathbf{0}$ & $(\mathbf{0 . 0})$ & 1 & $(0.9)$ & 1 & $(0.9)$ & 1 & $(0.8)$ \\
\hline Intervertebral disc protrusion & 0 & $(0.0)$ & 1 & $(0.9)$ & 0 & $(0.0)$ & 1 & $(0.8)$ \\
\hline Myositis & 0 & $(0.0)$ & 0 & $(0.0)$ & 1 & $(0.9)$ & 0 & $(0.0)$ \\
\hline $\begin{array}{l}\text { Neoplasms benign, malignant } \\
\text { and unspecified (incl cysts and } \\
\text { polyps) }\end{array}$ & 0 & $(0.0)$ & 1 & $(0.9)$ & $\mathbf{0}$ & $(0.0)$ & 1 & $(0.8)$ \\
\hline Prostate cancer & 0 & $(0.0)$ & 1 & $(0.9)$ & 0 & $(0.0)$ & 1 & $(0.8)$ \\
\hline Nervous system disorders & $\mathbf{0}$ & $(0.0)$ & 1 & $(0.9)$ & 3 & $(2.7)$ & 1 & $(0.8)$ \\
\hline $\begin{array}{l}\text { Depressed level of } \\
\text { consciousness }\end{array}$ & 0 & $(0.0)$ & 0 & $(0.0)$ & 1 & $(0.9)$ & 0 & $(0.0)$ \\
\hline Dizziness & 0 & $(0.0)$ & 0 & $(0.0)$ & 1 & $(0.9)$ & 0 & $(0.0)$ \\
\hline Headache & 0 & $(0.0)$ & 0 & $(0.0)$ & 1 & $(0.9)$ & 0 & $(0.0)$ \\
\hline Presyncope & 0 & $(0.0)$ & 1 & $(0.9)$ & 0 & $(0.0)$ & 1 & $(0.8)$ \\
\hline Renal and urinary disorders & $\mathbf{0}$ & $(\mathbf{0 . 0})$ & $\mathbf{0}$ & $(\mathbf{0 . 0})$ & 1 & $(0.9$ & $\mathbf{0}$ & $(\mathbf{0 . 0})$ \\
\hline Renal failure chronic & 0 & $(0.0)$ & 0 & $(0.0)$ & 1 & $(0.9)$ & 0 & $(0.0)$ \\
\hline $\begin{array}{l}\text { Respiratory, thoracic and } \\
\text { mediastinal disorders }\end{array}$ & $\mathbf{0}$ & $(0.0)$ & 2 & (1.8) & 1 & $(0.9)$ & 2 & (1.6) \\
\hline Acute respiratory failure & 0 & $(0.0)$ & 1 & $(0.9)$ & 0 & $(0.0)$ & 1 & $(0.8)$ \\
\hline Pleural effusion & 0 & $(0.0)$ & 1 & $(0.9)$ & 1 & $(0.9)$ & 1 & $(0.8)$ \\
\hline Vascular disorders & $\mathbf{0}$ & $(0.0)$ & 4 & (3.6) & 5 & $(4.4)$ & 4 & (3.3) \\
\hline Aortic aneurysm & 0 & $(0.0)$ & 0 & $(0.0)$ & 2 & $(1.8)$ & 0 & $(0.0)$ \\
\hline Extremity necrosis & 0 & $(0.0)$ & 1 & $(0.9)$ & 0 & $(0.0)$ & 1 & $(0.8)$ \\
\hline
\end{tabular}




\begin{tabular}{|l|r|r|r|r|r|r|r|r|}
\hline Hypertension & 0 & $(0.0)$ & 2 & $(1.8)$ & 1 & $(0.9)$ & $(1.6)$ \\
\hline Hypertensive crisis & 0 & $(0.0)$ & 1 & $(0.9)$ & 0 & $(0.0)$ & 1 & $(0.8)$ \\
\hline Orthostatic hypotension & 0 & $(0.0)$ & 0 & $(0.0)$ & 1 & $(0.9)$ & 0 & $(0.0)$ \\
\hline Peripheral venous disease & 0 & $(0.0)$ & 0 & $(0.0)$ & 1 & $(0.9)$ & 0 & $(0.0)$ \\
\hline
\end{tabular}

Every subject is counted a single time for each applicable row and column. 
Supplementary Table 2. Liver function tests

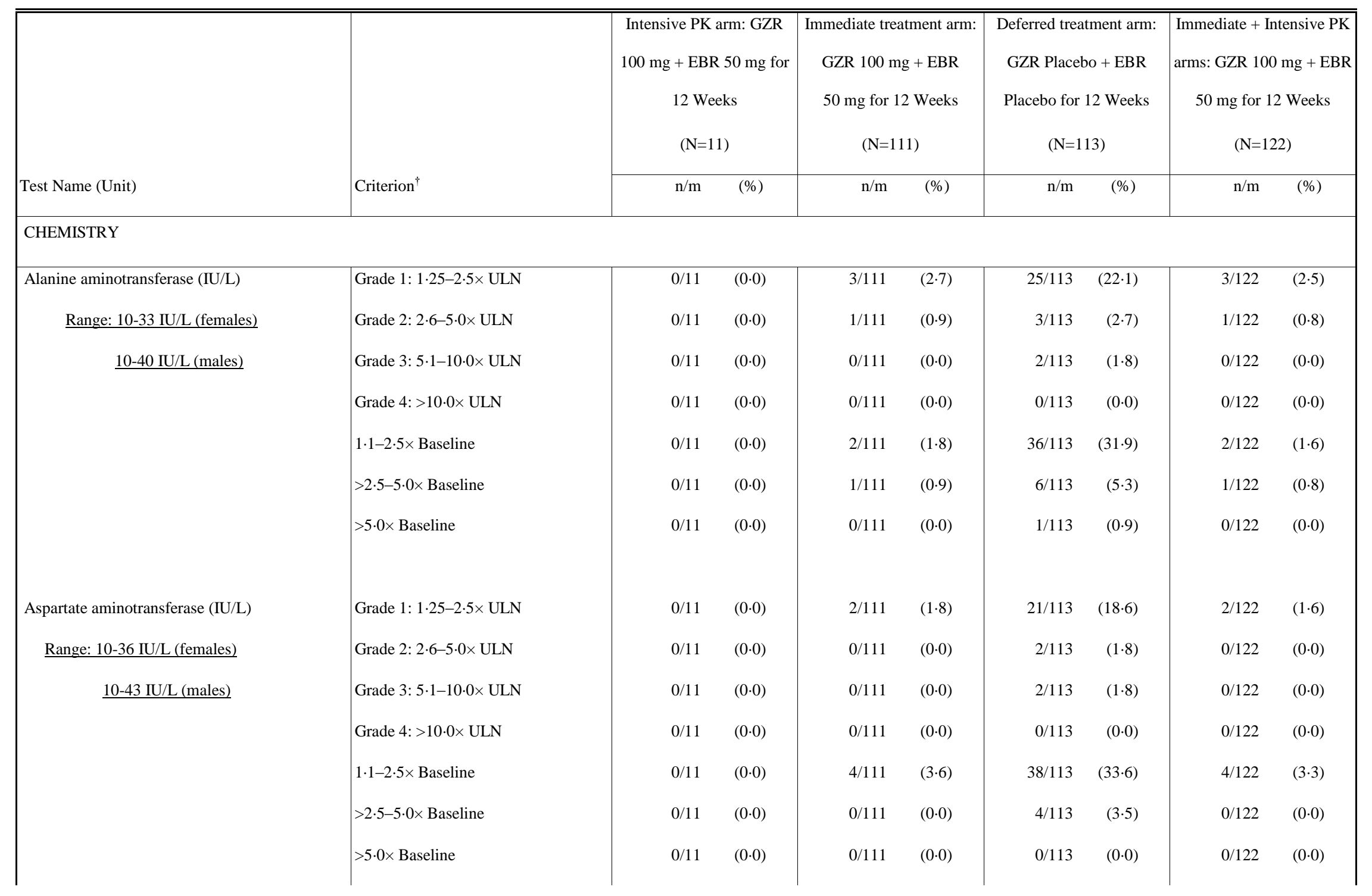




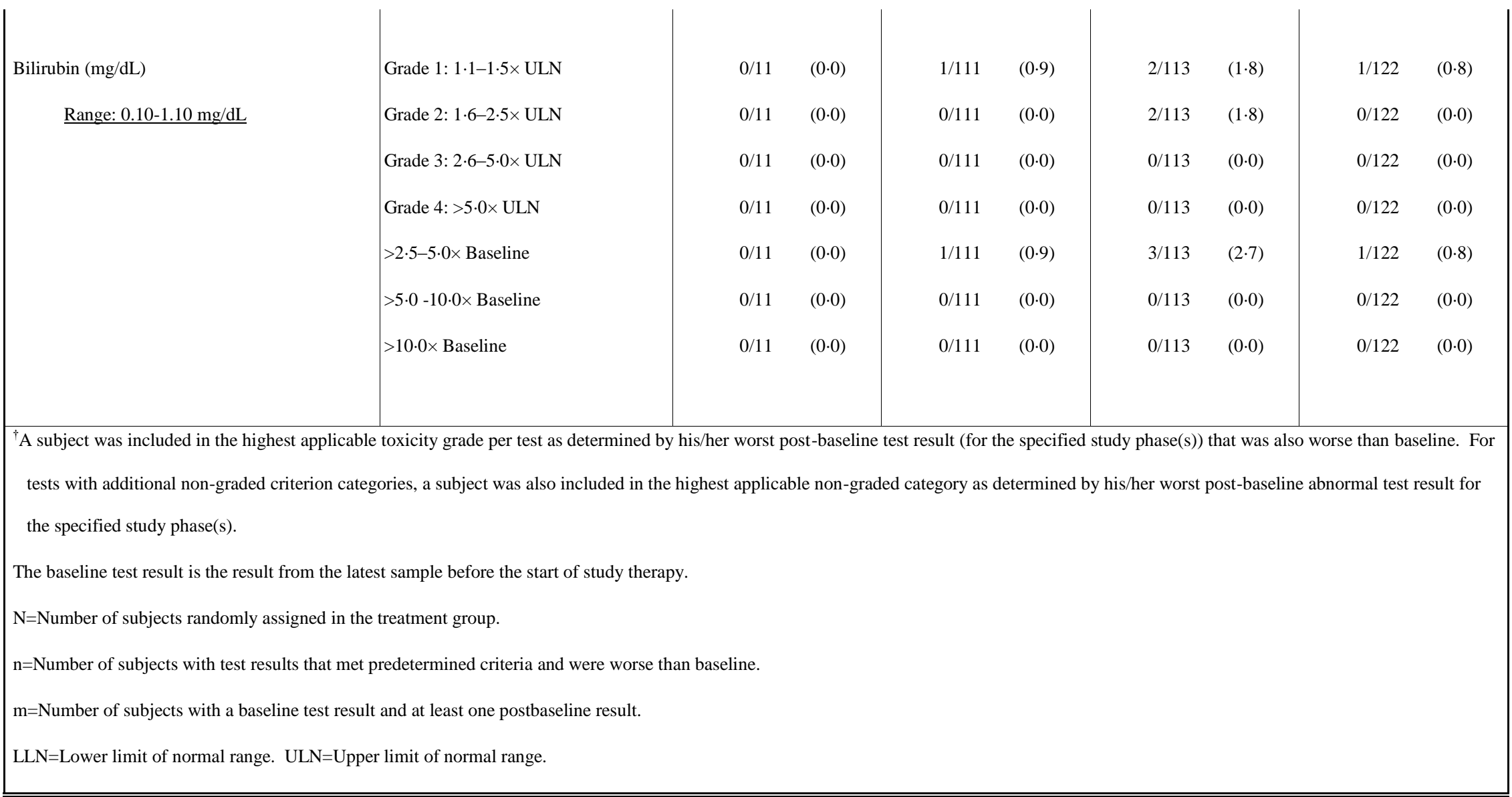


Supplementary Table 3. Laboratory safety summary

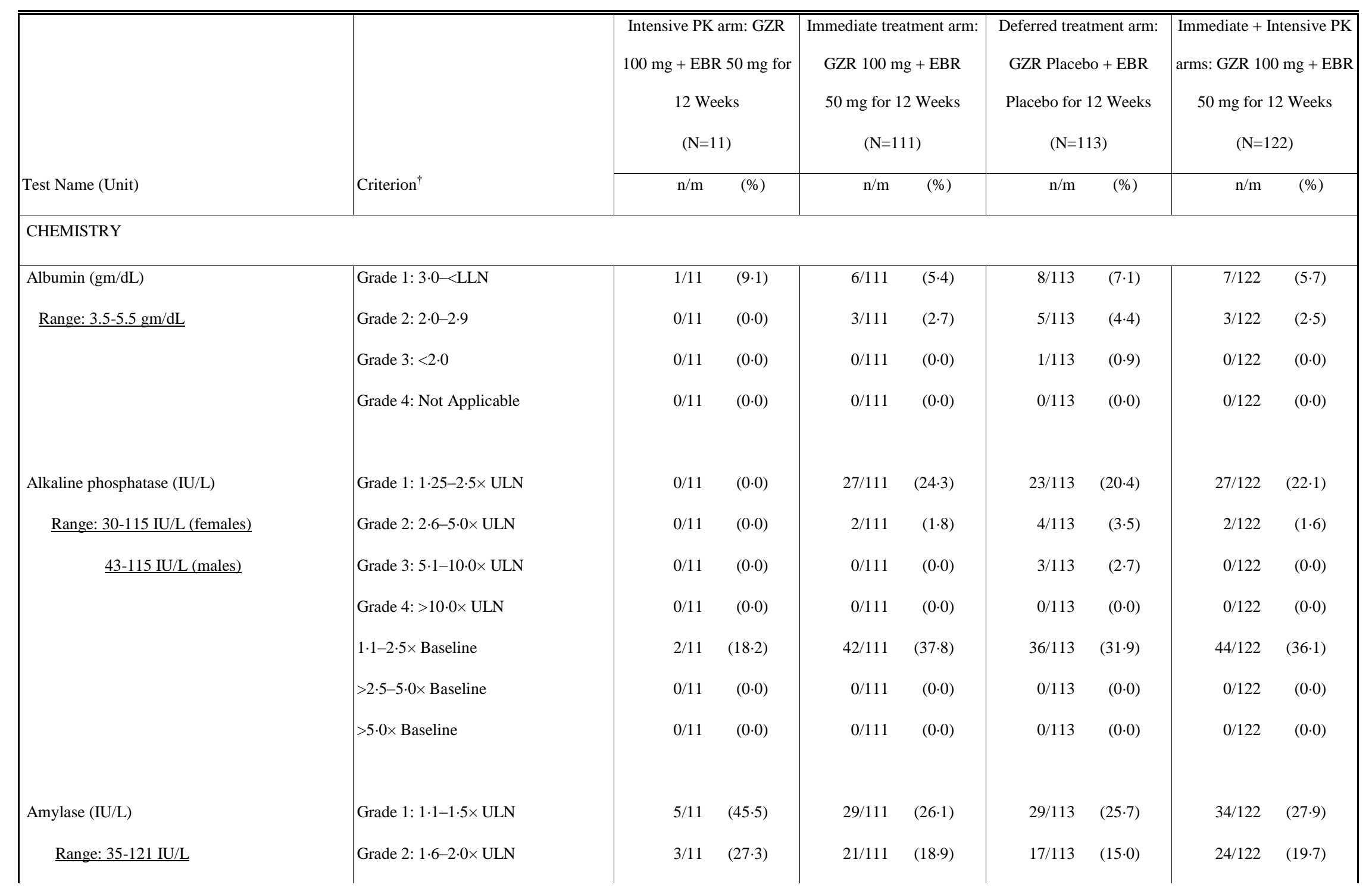




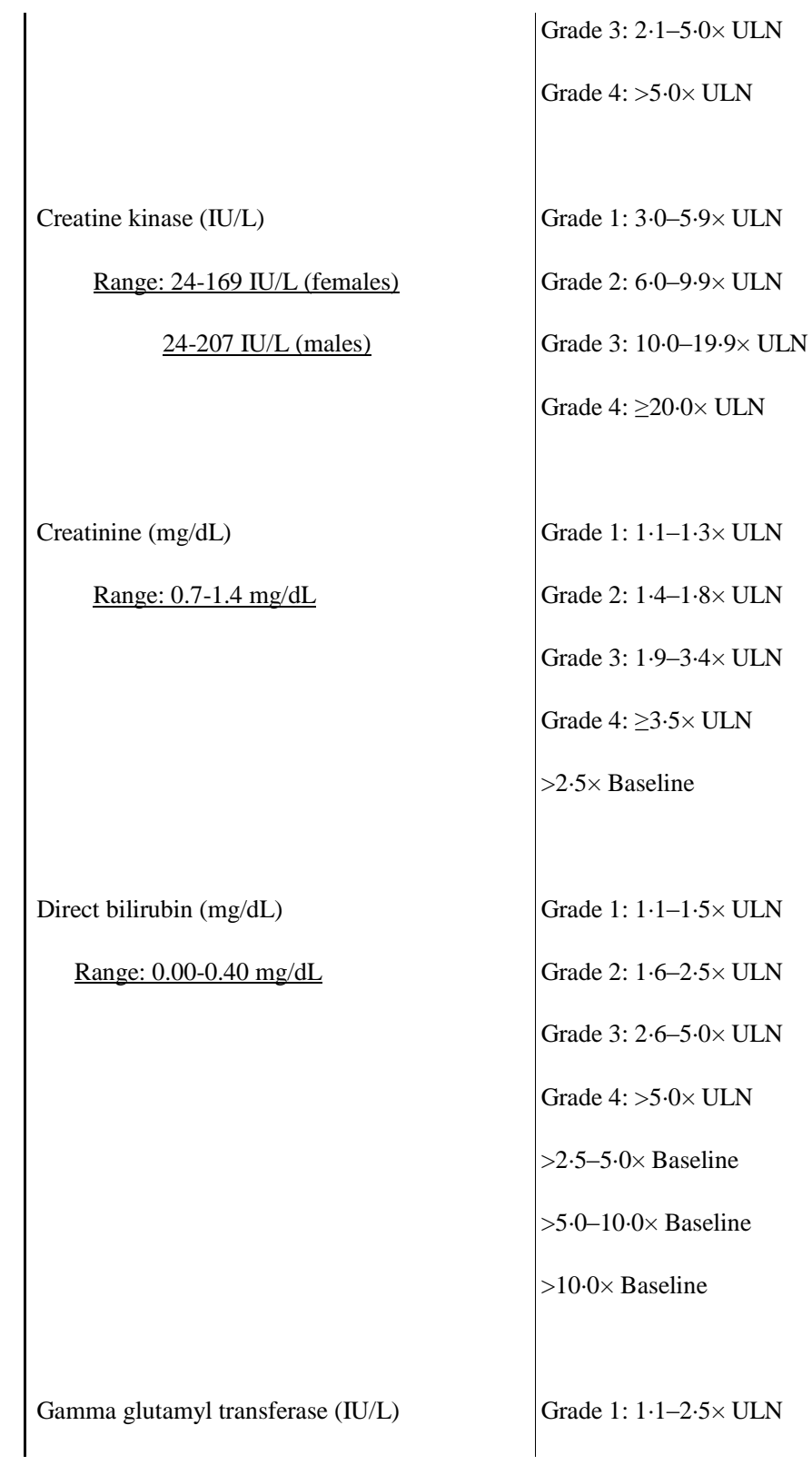

\begin{tabular}{|c|c|c|c|c|c|c|c|}
\hline $1 / 11$ & $(9 \cdot 1)$ & $24 / 111$ & $(21 \cdot 6)$ & $22 / 113$ & $(19 \cdot 5)$ & $25 / 122$ & $(20 \cdot 5)$ \\
\hline $0 / 11$ & $(0 \cdot 0)$ & $0 / 111$ & $(0 \cdot 0)$ & $4 / 113$ & $(3 \cdot 5)$ & $0 / 122$ & $(0 \cdot 0)$ \\
\hline $1 / 11$ & $(9 \cdot 1)$ & $6 / 111$ & (5.4) & $2 / 113$ & $(1 \cdot 8)$ & $7 / 122$ & $(5 \cdot 7)$ \\
\hline $1 / 11$ & $(9 \cdot 1)$ & $1 / 111$ & $(0 \cdot 9)$ & $1 / 113$ & $(0 \cdot 9)$ & $2 / 122$ & (1.6) \\
\hline $1 / 11$ & $(9 \cdot 1)$ & $0 / 111$ & $(0 \cdot 0)$ & $1 / 113$ & $(0 \cdot 9)$ & $1 / 122$ & $(0 \cdot 8)$ \\
\hline $0 / 11$ & $(0 \cdot 0)$ & $0 / 111$ & $(0 \cdot 0)$ & $1 / 113$ & $(0 \cdot 9)$ & $0 / 122$ & $(0 \cdot 0)$ \\
\hline $0 / 11$ & $(0 \cdot 0)$ & $0 / 111$ & $(0 \cdot 0)$ & $1 / 113$ & $(0 \cdot 9)$ & $0 / 122$ & $(0 \cdot 0)$ \\
\hline $0 / 11$ & $(0 \cdot 0)$ & $3 / 111$ & $(2 \cdot 7)$ & $3 / 113$ & $(2 \cdot 7)$ & $3 / 122$ & $(2 \cdot 5)$ \\
\hline $4 / 11$ & $(36 \cdot 4)$ & $14 / 111$ & $(12 \cdot 6)$ & $19 / 113$ & $(16 \cdot 8)$ & $18 / 122$ & $(14.8)$ \\
\hline $4 / 11$ & $(36 \cdot 4)$ & $74 / 111$ & $(66 \cdot 7)$ & $76 / 113$ & $(67 \cdot 3)$ & $78 / 122$ & (63.9) \\
\hline $0 / 11$ & $(0 \cdot 0)$ & $1 / 111$ & $(0 \cdot 9)$ & $0 / 113$ & $(0 \cdot 0)$ & $1 / 122$ & $(0 \cdot 8)$ \\
\hline $0 / 11$ & $(0 \cdot 0)$ & $1 / 111$ & $(0 \cdot 9)$ & $3 / 113$ & $(2 \cdot 7)$ & $1 / 122$ & $(0 \cdot 8)$ \\
\hline $0 / 11$ & $(0 \cdot 0)$ & $0 / 111$ & $(0 \cdot 0)$ & $3 / 113$ & $(2 \cdot 7)$ & $0 / 122$ & $(0 \cdot 0)$ \\
\hline $0 / 11$ & $(0 \cdot 0)$ & $0 / 111$ & $(0 \cdot 0)$ & $1 / 113$ & $(0 \cdot 9)$ & $0 / 122$ & $(0 \cdot 0)$ \\
\hline $0 / 11$ & $(0 \cdot 0)$ & $0 / 111$ & $(0 \cdot 0)$ & $0 / 113$ & $(0 \cdot 0)$ & $0 / 122$ & $(0 \cdot 0)$ \\
\hline $0 / 11$ & $(0 \cdot 0)$ & $0 / 111$ & $(0 \cdot 0)$ & $2 / 113$ & $(1 \cdot 8)$ & $0 / 122$ & $(0 \cdot 0)$ \\
\hline $0 / 11$ & $(0 \cdot 0)$ & $0 / 111$ & $(0 \cdot 0)$ & $1 / 113$ & $(0 \cdot 9)$ & $0 / 122$ & $(0 \cdot 0)$ \\
\hline $0 / 11$ & $(0 \cdot 0)$ & $0 / 111$ & $(0 \cdot 0)$ & $0 / 113$ & $(0 \cdot 0)$ & $0 / 122$ & $(0 \cdot 0)$ \\
\hline $0 / 11$ & $(0 \cdot 0)$ & $8 / 111$ & $(7 \cdot 2)$ & $33 / 113$ & $(29 \cdot 2)$ & $8 / 122$ & $(6 \cdot 6)$ \\
\hline
\end{tabular}




\begin{tabular}{|c|c|c|c|c|c|c|c|c|c|}
\hline Range: 5-32 IU/L (females) & Grade $2: 2 \cdot 6-5 \cdot 0 \times$ ULN & $0 / 11$ & $(0 \cdot 0)$ & $3 / 111$ & $(2 \cdot 7)$ & $8 / 113$ & $(7 \cdot 1)$ & $3 / 122$ & $(2 \cdot 5)$ \\
\hline \multirow[b]{3}{*}{ Triacylglycerol lipase (IU/L) } & Grade $3: 5 \cdot 1-20 \cdot 0 \times$ ULN & $0 / 11$ & $(0 \cdot 0)$ & $1 / 111$ & $(0 \cdot 9)$ & $13 / 113$ & $(11.5)$ & $1 / 122$ & $(0 \cdot 8)$ \\
\hline & Grade 4: $>20 \cdot 0 \times$ ULN & $0 / 11$ & $(0 \cdot 0)$ & $0 / 111$ & $(0 \cdot 0)$ & $4 / 113$ & $(3 \cdot 5)$ & $0 / 122$ & $(0 \cdot 0)$ \\
\hline & Grade $1: 1 \cdot 1-1 \cdot 5 \times$ ULN & $0 / 11$ & $(0 \cdot 0)$ & $25 / 111$ & $(22 \cdot 5)$ & $17 / 113$ & $(15 \cdot 0)$ & $25 / 122$ & $(20 \cdot 5)$ \\
\hline \multirow[t]{3}{*}{ Range: $13-60$ IU/L } & Grade $2: 1 \cdot 6-3 \cdot 0 \times$ ULN & $6 / 11$ & $(54 \cdot 5)$ & $40 / 111$ & $(36 \cdot 0)$ & $41 / 113$ & $(36 \cdot 3)$ & $46 / 122$ & $(37 \cdot 7)$ \\
\hline & Grade $3: 3 \cdot 1-5 \cdot 0 \times$ ULN & $1 / 11$ & $(9 \cdot 1)$ & $10 / 111$ & $(9 \cdot 0)$ & $15 / 113$ & $(13 \cdot 3)$ & $11 / 122$ & $(9 \cdot 0)$ \\
\hline & Grade 4: $>5.0 \times$ ULN & $0 / 11$ & $(0 \cdot 0)$ & $9 / 111$ & $(8 \cdot 1)$ & $4 / 113$ & $(3 \cdot 5)$ & $9 / 122$ & (7.4) \\
\hline \multicolumn{10}{|l|}{ COAGULATION } \\
\hline Prothrombin intl. normalized ratio & Grade 1: $1 \cdot 1-1 \cdot 5 \times$ ULN & $1 / 11$ & $(9 \cdot 1)$ & $16 / 111$ & $(14 \cdot 4)$ & $7 / 113$ & $(6 \cdot 2)$ & $17 / 122$ & $(13.9)$ \\
\hline \multirow[t]{4}{*}{ Range: 0.9-1.1 } & Grade 2: $1 \cdot 6-2 \cdot 0 \times$ ULN & $0 / 11$ & $(0 \cdot 0)$ & $2 / 111$ & $(1.8)$ & $1 / 113$ & $(0 \cdot 9)$ & $2 / 122$ & $(1 \cdot 6)$ \\
\hline & Grade $3: 2 \cdot 1-3 \cdot 0 \times$ ULN & $0 / 11$ & $(0 \cdot 0)$ & $2 / 111$ & $(1 \cdot 8)$ & $2 / 113$ & $(1 \cdot 8)$ & $2 / 122$ & $(1 \cdot 6)$ \\
\hline & Grade 4: $>3.0 \times$ ULN & $0 / 11$ & $(0 \cdot 0)$ & $0 / 111$ & $(0 \cdot 0)$ & $3 / 113$ & $(2 \cdot 7)$ & $0 / 122$ & $(0 \cdot 0)$ \\
\hline & $>1.5 \times$ Baseline & $0 / 11$ & $(0 \cdot 0)$ & $6 / 111$ & $(5 \cdot 4)$ & $7 / 113$ & $(6 \cdot 2)$ & $6 / 122$ & $(4 \cdot 9)$ \\
\hline \multicolumn{10}{|l|}{ HEMATOLOGY } \\
\hline Eosinophils/leukocytes (\%) & $>5 \%$ and baseline $<5 \%$ & $5 / 11$ & $(45 \cdot 5)$ & $21 / 111$ & $(18 \cdot 9)$ & $20 / 108$ & $(18 \cdot 5)$ & $26 / 122$ & $(21 \cdot 3)$ \\
\hline Range: $0.0-7.0 \%$ & $>5 \%$ and baseline $\geq 5 \%$ & $0 / 11$ & $(0 \cdot 0)$ & $19 / 111$ & $(17 \cdot 1)$ & $14 / 108$ & $(13 \cdot 0)$ & $19 / 122$ & $(15 \cdot 6)$ \\
\hline Hemoglobin $(\mathrm{g} / \mathrm{dL})$ & Grade 1: $10 \cdot 0-10 \cdot 9$ & $2 / 11$ & $(18 \cdot 2)$ & $24 / 111$ & $(21 \cdot 6)$ & $26 / 113$ & $(23 \cdot 0)$ & $26 / 122$ & $(21 \cdot 3)$ \\
\hline Range: $12.5-17.0 \mathrm{~g} / \mathrm{dL}$ & Grade 2: 9.0-9.9 & $3 / 11$ & $(27 \cdot 3)$ & $20 / 111$ & $(18 \cdot 0)$ & $16 / 113$ & $(14 \cdot 2)$ & $23 / 122$ & $(18.9)$ \\
\hline
\end{tabular}




\begin{tabular}{|c|c|}
\hline & $\begin{array}{l}\text { Grade } 3: 7 \cdot 0-8.9 \\
\text { Grade } 4:<7 \cdot 0 \\
8 \cdot 5-<10 \cdot 0 \\
<8.5\end{array}$ \\
\hline Leukocytes $\left(10^{3} / \mu \mathrm{L}\right)$ & Grade $1: 2 \cdot 0-2 \cdot 5$ \\
\hline Range: $3.5-12.5 \times 10^{3} / \mu \mathrm{L}$ & $\begin{array}{l}\text { Grade 2: } 1 \cdot 5-1.999 \\
\text { Grade 3: } 1 \cdot 0-1 \cdot 499 \\
\text { Grade 4: }<1 \cdot 0\end{array}$ \\
\hline Lymphocytes $\left(10^{3} / \mu \mathrm{L}\right)$ & Grade 1: $0 \cdot 60-0 \cdot 65$ \\
\hline Range: $0.9-3.6 \times 10^{3} / \mu \mathrm{L}$ & $\begin{array}{l}\text { Grade 2: } 0.50-0.599 \\
\text { Grade 3: 0.35-0.499 } \\
\text { Grade 4: }<0.35\end{array}$ \\
\hline Neutrophils $\left(10^{3} / \mu \mathrm{L}\right)$ & Grade 1: $1.00-1 \cdot 3$ \\
\hline Range: $1.7-7.9 \times 10^{3} / \mu \mathrm{L}$ & $\begin{array}{l}\text { Grade 2: } 0.75-0.999 \\
\text { Grade 3: } 0.50-0.749 \\
\text { Grade 4: }<0.50\end{array}$ \\
\hline Platelet $\left(10^{3} / \mu \mathrm{L}\right)$ & Grade 1: $100-124.999$ \\
\hline Range: $125-375 \times 10^{3} / \mu \mathrm{L}$ & $\begin{array}{l}\text { Grade 2: 50-99.999 } \\
\text { Grade 3: 25-49.999 }\end{array}$ \\
\hline
\end{tabular}

\begin{tabular}{|c|c|c|c|c|c|c|c|}
\hline $1 / 11$ & $(9 \cdot 1)$ & $11 / 111$ & $(9 \cdot 9)$ & $6 / 113$ & $(5 \cdot 3)$ & $12 / 122$ & $(9 \cdot 8)$ \\
\hline $0 / 11$ & $(0 \cdot 0)$ & $1 / 111$ & $(0 \cdot 9)$ & $2 / 113$ & $(1 \cdot 8)$ & $1 / 122$ & $(0 \cdot 8)$ \\
\hline $3 / 11$ & $(27 \cdot 3)$ & $27 / 111$ & $(24 \cdot 3)$ & $19 / 113$ & $(16 \cdot 8)$ & $30 / 122$ & (24.6) \\
\hline $1 / 11$ & $(9 \cdot 1)$ & $5 / 111$ & $(4 \cdot 5)$ & $5 / 113$ & $(4 \cdot 4)$ & $6 / 122$ & $(4.9)$ \\
\hline $0 / 11$ & $(0 \cdot 0)$ & $0 / 111$ & $(0 \cdot 0)$ & $1 / 113$ & $(0 \cdot 9)$ & $0 / 122$ & $(0 \cdot 0)$ \\
\hline $0 / 11$ & $(0 \cdot 0)$ & $0 / 111$ & $(0 \cdot 0)$ & $1 / 113$ & $(0 \cdot 9)$ & $0 / 122$ & $(0 \cdot 0)$ \\
\hline $0 / 11$ & $(0 \cdot 0)$ & $0 / 111$ & $(0 \cdot 0)$ & $0 / 113$ & $(0 \cdot 0)$ & $0 / 122$ & $(0 \cdot 0)$ \\
\hline $0 / 11$ & $(0 \cdot 0)$ & $0 / 111$ & $(0 \cdot 0)$ & $0 / 113$ & $(0 \cdot 0)$ & $0 / 122$ & $(0 \cdot 0)$ \\
\hline $0 / 11$ & $(0.0)$ & $5 / 111$ & $(4 \cdot 5)$ & $4 / 109$ & $(3 \cdot 7)$ & $5 / 122$ & $(4 \cdot 1)$ \\
\hline $0 / 11$ & $(0 \cdot 0)$ & $2 / 111$ & $(1 \cdot 8)$ & $4 / 109$ & $(3 \cdot 7)$ & $2 / 122$ & $(1 \cdot 6)$ \\
\hline $0 / 11$ & $(0 \cdot 0)$ & $0 / 111$ & $(0 \cdot 0)$ & 0/109 & $(0 \cdot 0)$ & $0 / 122$ & $(0 \cdot 0)$ \\
\hline $0 / 11$ & $(0 \cdot 0)$ & $0 / 111$ & $(0 \cdot 0)$ & 0/109 & $(0 \cdot 0)$ & $0 / 122$ & $(0 \cdot 0)$ \\
\hline $0 / 11$ & $(0 \cdot 0)$ & $1 / 111$ & $(0 \cdot 9)$ & $2 / 109$ & $(1 \cdot 8)$ & $1 / 122$ & $(0 \cdot 8)$ \\
\hline $0 / 11$ & $(0 \cdot 0)$ & 1/111 & $(0 \cdot 9)$ & $1 / 109$ & $(0 \cdot 9)$ & $1 / 122$ & $(0 \cdot 8)$ \\
\hline $0 / 11$ & $(0 \cdot 0)$ & $0 / 111$ & $(0 \cdot 0)$ & $1 / 109$ & $(0 \cdot 9)$ & $0 / 122$ & $(0 \cdot 0)$ \\
\hline $0 / 11$ & $(0 \cdot 0)$ & 0/111 & $(0 \cdot 0)$ & $0 / 109$ & $(0 \cdot 0)$ & $0 / 122$ & $(0 \cdot 0)$ \\
\hline $2 / 11$ & $(18 \cdot 2)$ & $10 / 110$ & $(9 \cdot 1)$ & $15 / 113$ & $(13 \cdot 3)$ & $12 / 121$ & $(9 \cdot 9)$ \\
\hline $0 / 11$ & $(0 \cdot 0)$ & $8 / 110$ & $(7 \cdot 3)$ & $12 / 113$ & $(10 \cdot 6)$ & $8 / 121$ & $(6 \cdot 6)$ \\
\hline $0 / 11$ & $(0 \cdot 0)$ & $0 / 110$ & $(0.0)$ & $0 / 113$ & $(0 \cdot 0)$ & $0 / 121$ & $(0 \cdot 0)$ \\
\hline
\end{tabular}




\section{Grade 4: $<25$}

A subject was included in the highest applicable toxicity grade per test as determined by his/her worst post-baseline test result (for the specified study phase(s)) that was also worse than baseline. For tests with additional non-graded criterion categories, a subject was also included in the highest applicable non-graded category as determined by his/her worst post-baseline abnormal test result for the specified study phase(s)

The baseline test result is the result from the latest sample before the start of study therapy.

$\mathrm{N}=$ Number of subjects randomly assigned in the treatment group.

$\mathrm{n}=$ Number of subjects with test results that met predetermined criteria and were worse than baseline

$m=$ Number of subjects with a baseline test result and at least one postbaseline result.

LLN=Lower limit of normal range. ULN=Upper limit of normal range. 


\section{Supplementary Table 4. Special Renal Function Monitored Adverse Events}

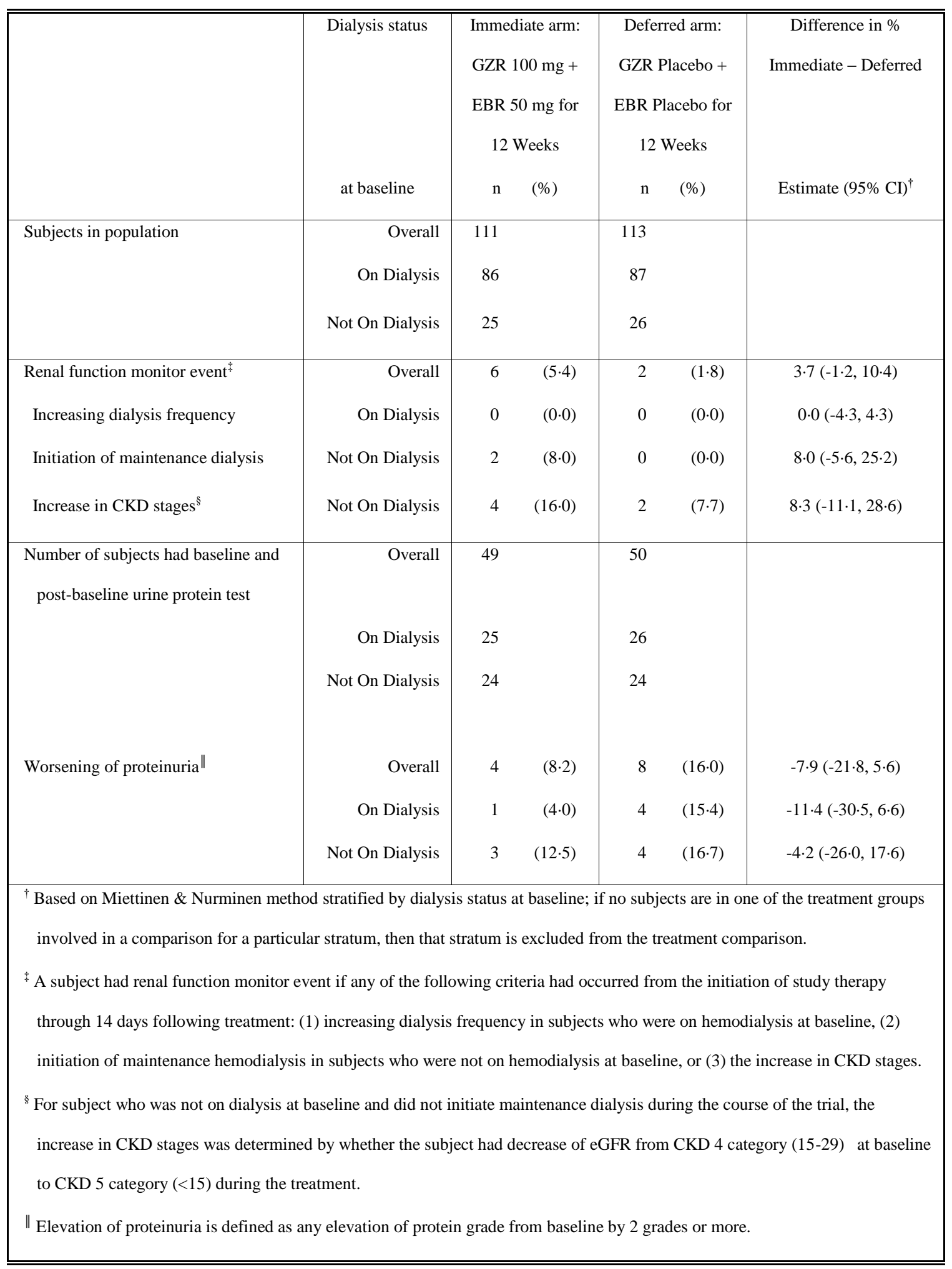


Supplementary Table 5. Change from baseline in eGFR $\left(\mathrm{mL} / \mathrm{min} / 1 \cdot 73 \mathrm{~m}^{2}\right)$ initial treatment

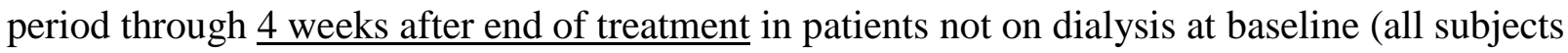
as treated)

\begin{tabular}{|c|c|c|c|c|c|}
\hline \multirow[b]{2}{*}{ Visit Time Point } & \multicolumn{2}{|c|}{$\begin{array}{c}\text { Immediate treatment arm: GZR } \\
100 \mathrm{mg}+\text { EBR } 50 \mathrm{mg} \text { for } 12 \text { Weeks }\end{array}$} & \multicolumn{2}{|c|}{$\begin{array}{c}\text { Deferred treatment arm: GZR Placebo } \\
+ \text { EBR Placebo for } 12 \text { Weeks }\end{array}$} & \multirow{2}{*}{$\begin{array}{c}\text { Difference in Change } \\
\text { from Baseline } \\
\text { (Immediate - Deferred } \\
\text { Arm) } \\
\text { Estimate (95\% CI) }\end{array}$} \\
\hline & $\mathrm{N}$ & $\begin{array}{c}\text { Change from Baseline } \\
\text { Mean (SD) }\end{array}$ & $\mathrm{N}$ & $\begin{array}{c}\text { Change from Baseline } \\
\text { Mean (SD) }\end{array}$ & \\
\hline TW1 & 23 & $0.04(2 \cdot 06)$ & 26 & $-1 \cdot 54(3 \cdot 22)$ & $1.58(0 \cdot 01,3 \cdot 16)$ \\
\hline TW2 & 23 & $0 \cdot 22(3 \cdot 55)$ & 26 & $-1 \cdot 31(3 \cdot 17)$ & $1 \cdot 53(-0 \cdot 41,3 \cdot 46)$ \\
\hline TW3 & 23 & $-0.04(4.49)$ & 25 & $-1.04(3.02)$ & $1.00(-1 \cdot 21,3 \cdot 20)$ \\
\hline TW4 & 24 & $-0 \cdot 42(2 \cdot 21)$ & 23 & $-1 \cdot 30(2.75)$ & $0 \cdot 89(-0 \cdot 57,2 \cdot 35)$ \\
\hline TW6 & 25 & $-0 \cdot 36(5 \cdot 75)$ & 26 & $-2 \cdot 08(3 \cdot 11)$ & $1 \cdot 72(-0.87,4 \cdot 30)$ \\
\hline TW8 & 24 & $0 \cdot 88(4 \cdot 85)$ & 25 & $-1 \cdot 52(3 \cdot 28)$ & $2.40(0 \cdot 02,4 \cdot 77)$ \\
\hline TW10 & 25 & $-0 \cdot 24(4 \cdot 88)$ & 25 & $-0 \cdot 80(3 \cdot 15)$ & $0 \cdot 56(-1 \cdot 77,2 \cdot 89)$ \\
\hline TW12 & 25 & $-0 \cdot 32(4 \cdot 65)$ & 24 & $-1.08(3.83)$ & $0 \cdot 76(-1 \cdot 69,3 \cdot 22)$ \\
\hline FW4 & 24 & $-0.13(4.79)$ & 24 & $-1 \cdot 13(4 \cdot 85)$ & $1 \cdot 00(-1 \cdot 80,3 \cdot 80)$ \\
\hline
\end{tabular}


Supplementary Table 6. Change from baseline in serum creatinine $(\mathrm{mg} / \mathrm{dL})$ initial treatment

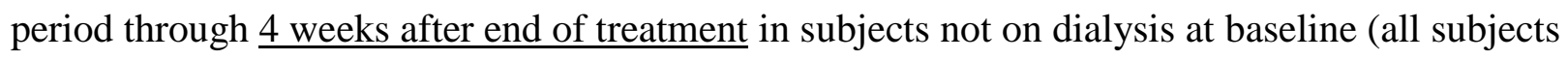
as treated)

\begin{tabular}{|c|c|c|c|c|c|}
\hline \multirow[b]{2}{*}{ Visit Time Point } & \multicolumn{2}{|c|}{$\begin{array}{c}\text { Immediate treatment arm: GZR } \\
100 \mathrm{mg}+\text { EBR } 50 \mathrm{mg} \text { for } 12 \text { Weeks }\end{array}$} & \multicolumn{2}{|c|}{$\begin{array}{l}\text { Deferred treatment arm: GZR Placebo } \\
+ \text { EBR Placebo for } 12 \text { Weeks }\end{array}$} & \multirow{2}{*}{$\begin{array}{l}\text { Difference in Change } \\
\text { from Baseline } \\
\text { (Immediate - Deferred } \\
\text { Arm) } \\
\text { Estimate }(95 \% \mathrm{CI})\end{array}$} \\
\hline & $\mathrm{N}$ & $\begin{array}{c}\text { Change from Baseline } \\
\text { Mean (SD) }\end{array}$ & $\mathrm{N}$ & $\begin{array}{c}\text { Change from Baseline } \\
\text { Mean (SD) }\end{array}$ & \\
\hline TW1 & 23 & $0.02(0 \cdot 31)$ & 26 & $0 \cdot 12(0 \cdot 31)$ & $-0.09(-0 \cdot 27,0 \cdot 08)$ \\
\hline TW2 & 23 & $-0 \cdot 04(0 \cdot 45)$ & 26 & $0.05(0 \cdot 33)$ & $-0 \cdot 10(-0 \cdot 32,0 \cdot 13)$ \\
\hline TW3 & 23 & $0.03(0.58)$ & 25 & $0 \cdot 05(0 \cdot 36)$ & $-0.03(-0 \cdot 31,0 \cdot 25)$ \\
\hline TW4 & 24 & $0.05(0.59)$ & 23 & $0 \cdot 10(0 \cdot 37)$ & $-0 \cdot 05(-0 \cdot 34,0 \cdot 24)$ \\
\hline TW6 & 25 & $0.24(1.09)$ & 26 & $0 \cdot 17(0 \cdot 37)$ & $0 \cdot 07(-0 \cdot 39,0 \cdot 52)$ \\
\hline TW8 & 24 & $-0 \cdot 19(0 \cdot 62)$ & 25 & $0 \cdot 10(0 \cdot 33)$ & $-0 \cdot 28(-0 \cdot 57,-0 \cdot 00)$ \\
\hline TW10 & 25 & $0.04(0 \cdot 85)$ & 25 & $0.09(0 \cdot 34)$ & $-0 \cdot 04(-0 \cdot 41,0 \cdot 33)$ \\
\hline TW12 & 25 & $0 \cdot 07(0 \cdot 96)$ & 24 & $0 \cdot 13(0 \cdot 35)$ & $-0 \cdot 06(-0 \cdot 48,0 \cdot 36)$ \\
\hline FW4 & 24 & $0.00(1.01)$ & 24 & $0 \cdot 18(0 \cdot 46)$ & $-0 \cdot 18(-0 \cdot 64,0 \cdot 28)$ \\
\hline
\end{tabular}


Protocol/Amendment No.: 052-04

\section{THIS PROTOCOL AMENDMENT AND ALL OF THE INFORMATION RELATING TO IT ARE CONFIDENTIAL AND PROPRIETARY PROPERTY OF MERCK SHARP \& DOHME CORP., A SUBSIDIARY OF MERCK \& CO., INC., WHITEHOUSE STATION, NJ, U.S.A.}

\section{SPONSOR:}

Merck Sharp \& Dohme Corp., a subsidiary of Merck \& Co., Inc.

(hereafter referred to as the Sponsor or Merck)

One Merck Drive

P.O. Box 100

Whitehouse Station, NJ 08889-0100, U.S.A.

Protocol-specific Sponsor Contact information can be found in the Investigator Trial File Binder (or equivalent).

\section{TITLE:}

A Phase II/III Randomized Clinical Trial to Study the Efficacy and Safety of the Combination Regimen of MK-5172 and MK-8742 in Subjects with Chronic Hepatitis C Virus Infection and Chronic Kidney Disease

IND NUMBER: [110,261]

EudraCT NUMBER: [2013-003858-25] 


\section{TABLE OF CONTENTS}

SUMMARY OF CHANGES ................................................................................................. 10

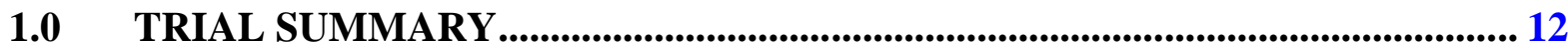

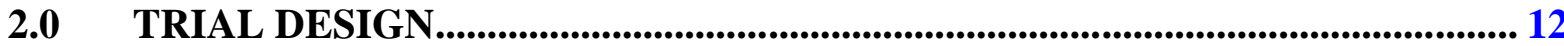

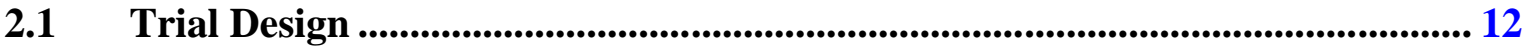

$2.2 \quad$ Trial Diagram................................................................................................................ 15

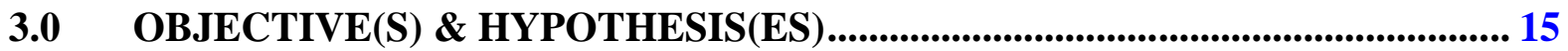

3.1 Primary Objective(s) \& Hypothesis(es) .................................................................. 15

3.2 Secondary Objective(s) \& Hypothesis(es)................................................................ 16

3.3 Other Objectives (e.g., Tertiary, Exploratory, etc.)................................................ 16

4.0 BACKGROUND \& RATIONALE ....................................................................... 17

4.1 Background .................................................................................................................... 17

4.1.1 Pharmaceutical and Therapeutic Background …………………..................... 17

4.1.2 Pre-clinical and Clinical Trials ......................................................................... 17

4.1.3 Ongoing Clinical Trials............................................................................. 18

4.2 Rationale ................................................................................................................... 19

4.2.1 Rationale for the Trial and Selected Subject Population ..................................... 19

4.2.2 Rationale for Dose Selection/Regimen/Modification ........................................... 23

4.2.3 Rationale for Endpoints .......................................................................... 24

4.2.3.1 Efficacy Endpoints ................................................................................... 24

4.2.3.1.1 Measurements of HCV RNA .......................................................... 24

4.2.3.1.1.1 Definition of Efficacy Endpoints ..................................................... 25

4.2.3.1.1.2 Definition of Virologic Failure: Virologic Breakthrough, NonResponse, Rebound and Relapse ......................................................... 25

4.2.3.1.2 Viral Resistance Measurements.......................................................... 26

4.2.3.1.3 IL28B, ADME genes and HLA Measurements ..................................... 26

4.2.3.1.4 Patient Reported Outcome Endpoint .................................................... 26

4.2.3.2 Safety Endpoints ....................................................................................... 27

4.2.3.3 Pharmacokinetic Endpoints ....................................................................... 27

4.2.3.4 Planned Exploratory Evaluation of Cryoglobulinemia .................................. 28 
4.2.3.5 Planned Exploratory Biomarker Research .............................................. 28

4.2.3.6 Future Biomedical Research .................................................................... 28

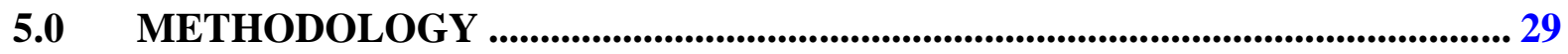

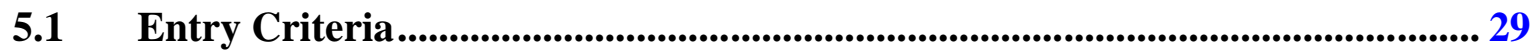

5.1.1 Diagnosis/Condition for Entry into the Trial ............................................... 29

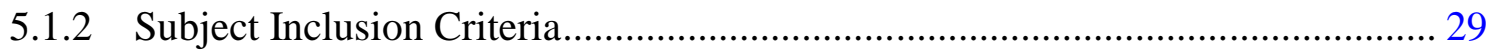

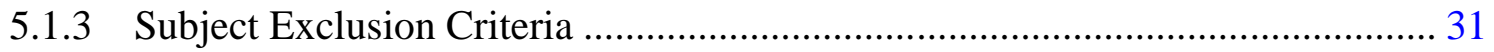

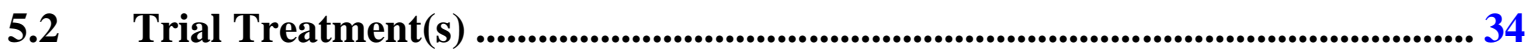

5.2.1 Dose Selection/Modification .................................................................. 34

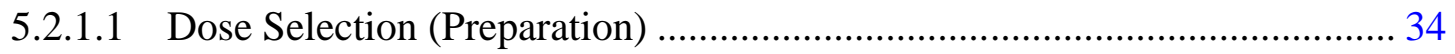

5.2.1.2 Dose Modification (Escalation/Titration/Other) ....................................... 34

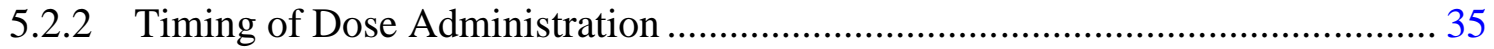

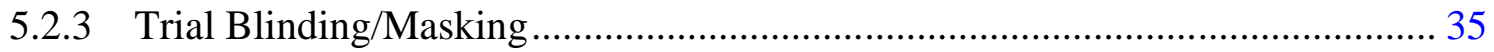

5.3 Randomization or Treatment Allocation ................................................................. 36

5.4 Stratification ................................................................................................. 36

5.5 Concomitant Medications/Vaccinations (Allowed \& Prohibited) .................... 36

5.6 Rescue Medications \& Supportive Care ............................................................. 39

5.7 Diet/Activity/Other Considerations............................................................ 39

5.8 Subject Withdrawal/Discontinuation Criteria .............................................. 39

5.9 Subject Replacement Strategy ............................................................... 41

5.10 Beginning and End of the Trial .............................................................. 41

5.11 Clinical Criteria for Early Trial Termination ............................................... 41

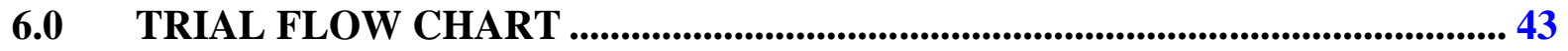

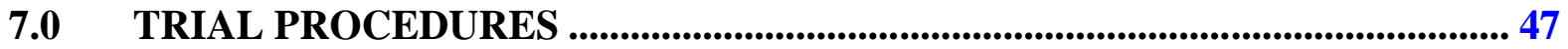

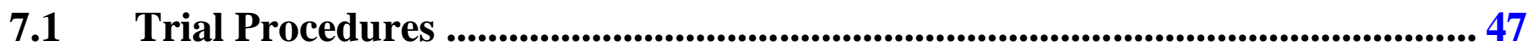

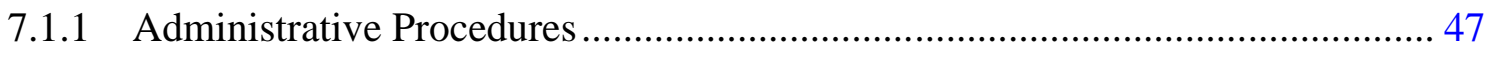

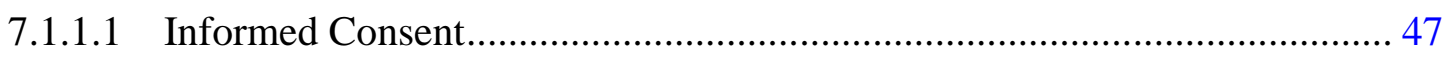

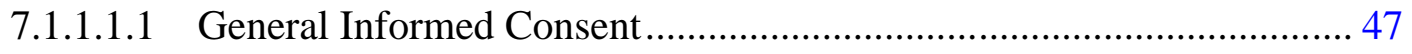

7.1.1.1.2 Consent and Collection of Specimens for Future Biomedical Research ................................................................................ 48

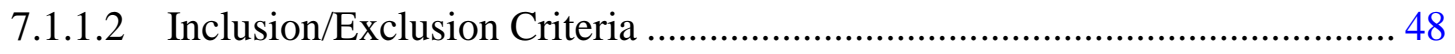

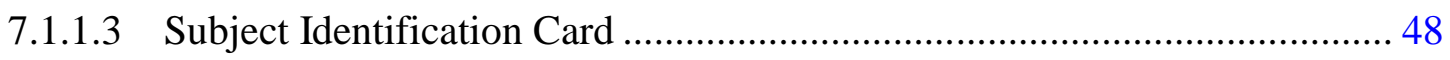


Protocol/Amendment No.: 052-04

7.1.1.4 Medical History ………………................................................................. 48

7.1.1.5 Prior and Concomitant Medications Review .............................................. 48

7.1.1.5.1 Prior Medications................................................................................ 48

7.1.1.5.2 Concomitant Medications .................................................................. 48

7.1.1.6 Assignment of Screening Number ............................................................. 48

7.1.1.7 Assignment of Randomization Number.......................................................... 49

7.1.1.8 Trial Compliance (Medication/Diet/Activity/Other) ..................................... 49

7.1.2 Clinical Procedures/Assessments..................................................................... 49

7.1.2.1 Physical Examination.................................................................................. 49

7.1.2.2 Weight and Height Assessment ................................................................... 50

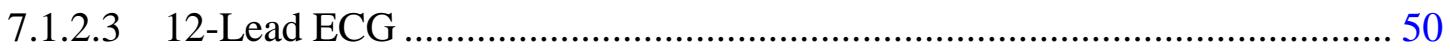

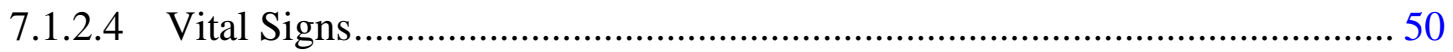

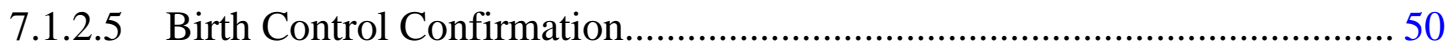

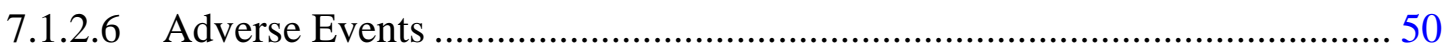

7.1.2.7 Noninvasive Methods of Cirrhosis Evaluation................................................ 51

7.1.3 Patient-Reported Outcomes ......................................................................... 51

7.1.4 Laboratory Procedures/Assessments ................................................................... 52

7.1.4.1 Laboratory Safety Evaluations (Hematology, Chemistry and Urinalysis) .. 52

7.1.4.2 Pharmacokinetic/Pharmacodynamic Evaluations ........................................... 53

7.1.4.2.1 Blood Collection for Plasma MK-5172 and MK-8742 ......................... 54

7.1.4.3 Future Biomedical Research.................................................................. 57

7.1.4.4 HCV Evaluation..................................................................................... 57

7.1.5 Other Procedures......................................................................................... 58

7.1.5.1 Withdrawal/Discontinuation .................................................................... 58

7.1.5.1.1 Withdrawal From Future Biomedical Research ……………................. 58

7.1.5.2 Blinding/Unblinding ............................................................................... 59

7.1.5.3 Calibration of Critical Equipment.............................................................. 59

7.1.5.4 Rescreening ............................................................................................... 59

7.1.5.5 PK Sampling Time Points.......................................................................... 59

7.1.6 Visit Requirements............................................................................................. 60

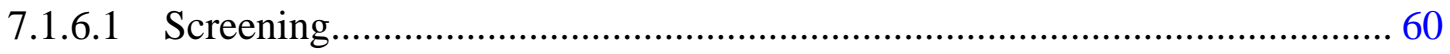

7.1.6.2 Treatment Period Visit .................................................................................. 60 


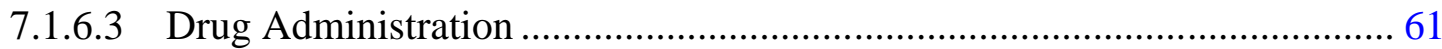

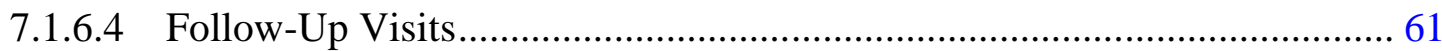

7.1.6.5 Evaluations of Laboratory Safety Signals...................................................... 62

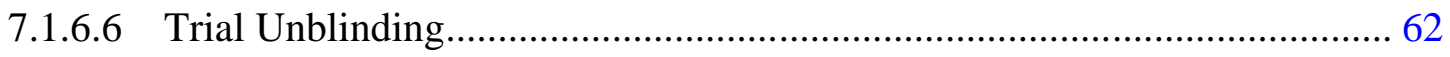

7.2 Assessing and Recording Adverse Events ........................................................... 63

7.2.1 Definition of an Overdose for This Protocol and Reporting of Overdose to

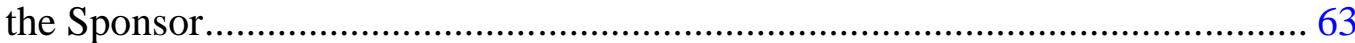

7.2.2 Reporting of Pregnancy and Lactation to the Sponsor ........................................ 64

7.2.3 Immediate Reporting of Adverse Events to the Sponsor ....................................... 64

7.2.3.1 Serious Adverse EventsAdverse Events and Incidents................................. 64

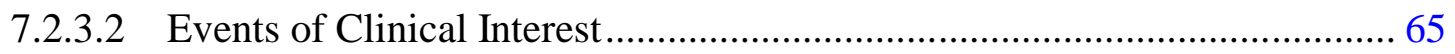

7.2.3.3 Protocol-Specific Exceptions to Serious Adverse Event Reporting ............. 65

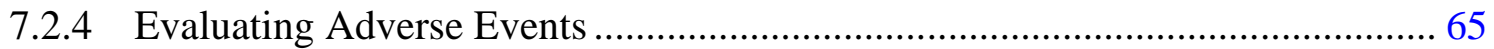

7.2.5 Sponsor Responsibility for Reporting Adverse Events ....................................... 68

7.3 TRIAL GOVERNANCE AND OVERSIGHT .................................................68

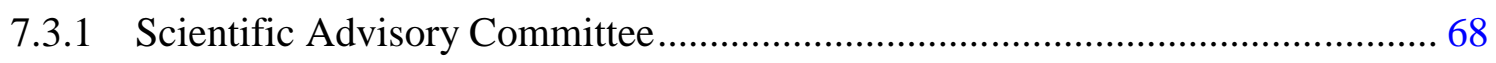

7.3.2 Data Monitoring Committee ............................................................................ 68

8.0 STATISTICAL ANALYSIS PLAN .......................................................................... 68

8.1 Statistical Analysis Plan Summary ......................................................................... 69

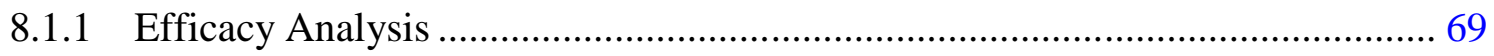

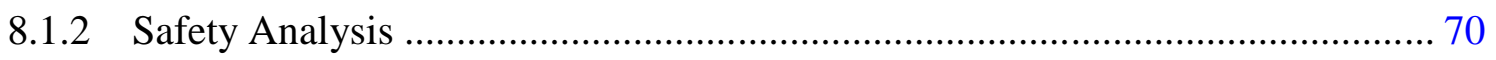

8.1.3 Power and Sample Size................................................................................. 70

8.2 Statistical Analysis Plan ...................................................................................... 71

8.2.1 Responsibility for Analyses/In-House Blinding ................................................. 71

8.2.2 Hypotheses/Estimation ................................................................................. 72

8.2.3 Analysis Endpoints ................................................................................ 72

8.2.3.1 Efficacy/Pharmacokinetic Endpoints......................................................... 72

8.2.3.1.1 Efficacy Endpoints ............................................................................. 72

8.2.3.1.2 Pharmacokinetic Endpoints .................................................................. 72

8.2.3.1.3 Exploratory Endpoints ............................................................................ 73

8.2.3.2 Safety Endpoints .................................................................................. 73

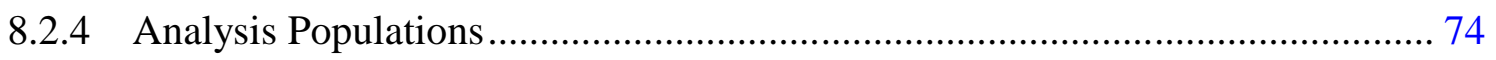


8.2.4.1 Efficacy Analysis Populations …............................................................. 74

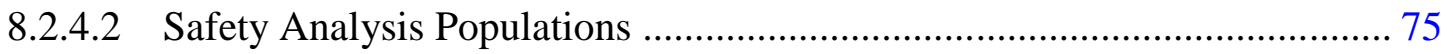

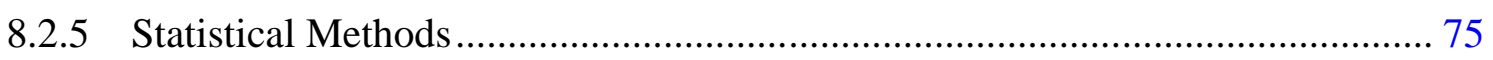

8.2.5.1 Statistical Methods for Efficacy Analyses ................................................. 76

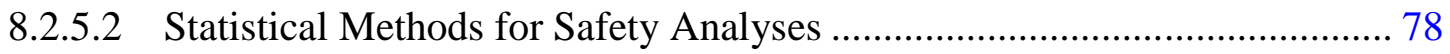

8.2.5.3 Summaries of Baseline Characteristics, Demographics, and Other

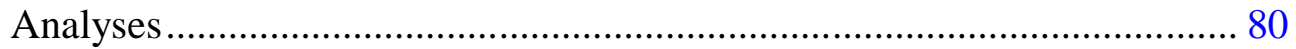

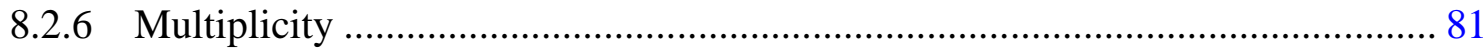

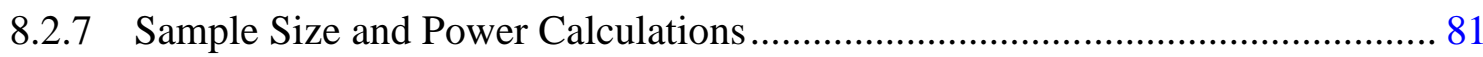

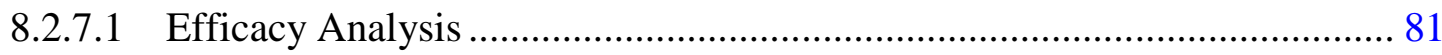

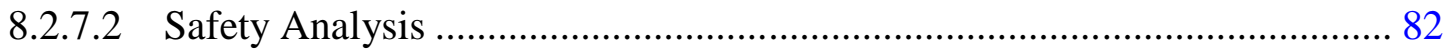

8.2.8 Subgroup Analyses and Effects of Baseline Factors ..................................... 83

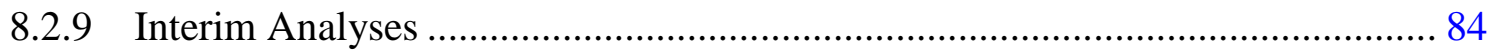

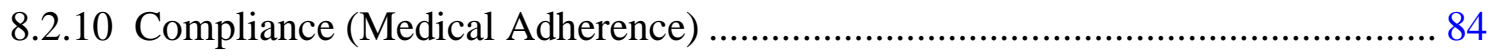

9.0 LABELING, PACKAGING, STORAGE AND RETURN OF CLINICAL SUPPLIES ............................................................................................................. 84

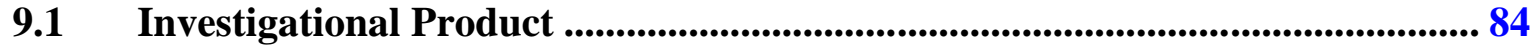

9.2 Packaging and Labeling Information .................................................................. 85

9.3 Clinical Supplies Disclosure ................................................................. 85

9.4 Storage and Handling Requirements ............................................................ 86

9.5 Returns and Reconciliation ......................................................................... 86

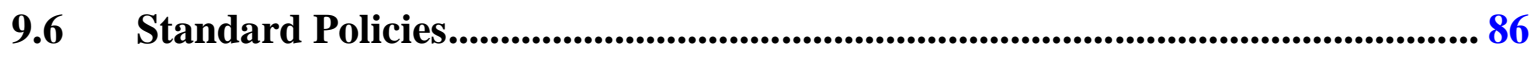

10.0 ADMINISTRATIVE AND REGULATORY DETAILS ................................... 86

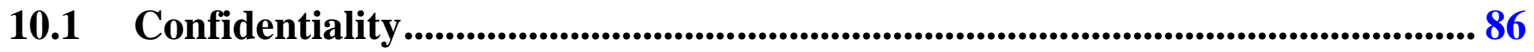

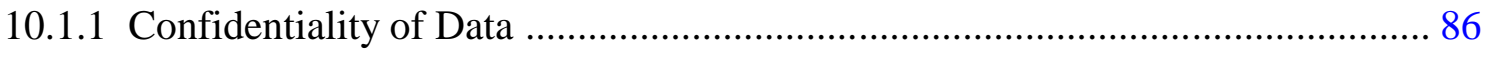

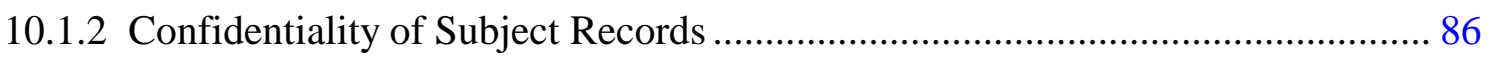

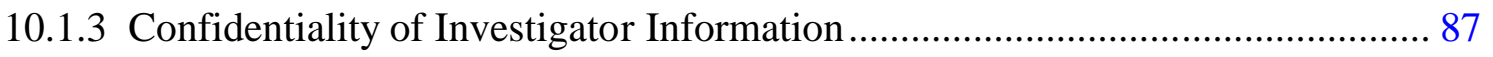

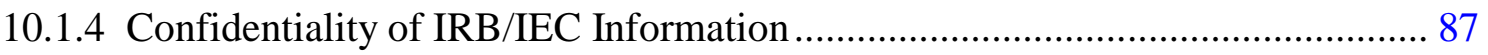

10.2 Compliance with Financial Disclosure Requirements .................................... 87

10.3 Compliance with Law, Audit and Debarment .............................................. 88

10.4 Compliance with Trial Registration and Results Posting Requirements ........ 90

10.5 Quality Management System ..................................................................... 90 
10.6 Data Management ................................................................................................... 90

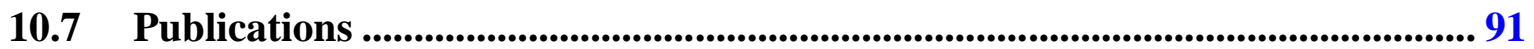

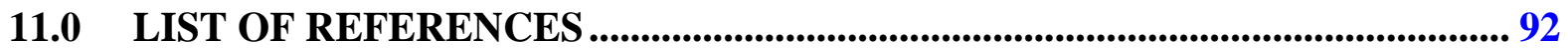

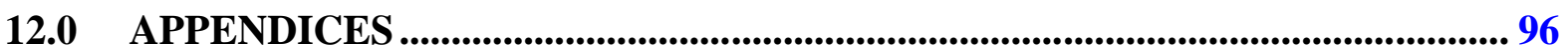

12.1 Merck Code of Conduct for Clinical Trials........................................................... 96

12.2 Collection and Management of Specimens for Future Biomedical

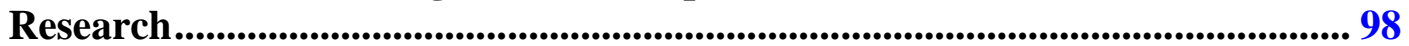

12.3 Understanding the Intent, Scope and Public Health Benefits of Exploratory Biomarker Research: A Guide for IRBs/IECs and Investigational Site Staff ................................................................................................ 104

12.4 List of Abbreviations and Definitions of Terms................................................. 115

12.5 Approximate Blood/Tissue Volumes Drawn/Collected by Trial Visit and by Sample Types ................................................................................................................ 117

12.6 Benefit/Risk for MK-5172 ............................................................................ 118

12.7 Benefit/Risk for MK-8742 ....................................................................................... 120

13.0 SIGNATURES.................................................................................................... 122

13.1 Sponsor's Representative .................................................................................... 122

13.2 Investigator ...................................................................................................................... 122 
Protocol/Amendment No.: 052-04

\section{LIST OF TABLES}

Table 1 Definition of HCV and CKD in Target Subject Population ........................................... 14

Table 2 Categories of Chronic Kidney Disease ............................................................................ 20

Table 3 Nomenclature for Describing HCV RNA Levels ............................................................ 25

Table $4 \quad$ Laboratory Exclusionary Values........................................................................................ 33

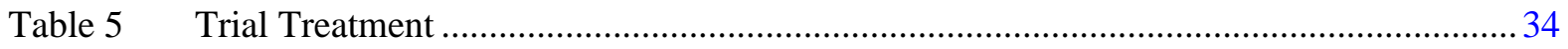

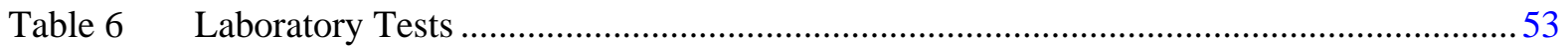

Table 7 Pharmacokinetic Sampling Timepoints- Population PK (All Subjects) .......................... 55

Table 8 Pharmacokinetic Sampling Timepoints- Intensive PK Arm ........................................... 56

Table 9 Pharmacokinetic Sampling Timepoints- Evening Pre-Dose PK Cohort.......................... 57

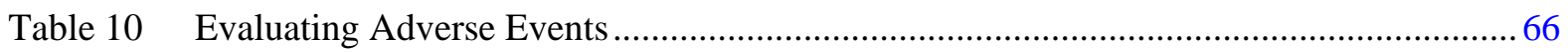

Table 11 Summary of Analysis Strategy for Key Efficacy Endpoints ...........................................69

Table 12 Analysis Strategy for Efficacy Endpoints..................................................................... 78

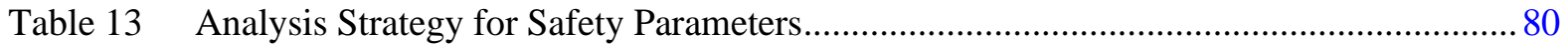

Table 14 Power Calculations for the Primary Hypothesis Test Within the Subjects of the Immediate Treatment and the Intensive PK Arms .......................................................... 82

Table 15 Power to Detect Difference in Adverse Event Rate....................................................... 82

Table 16 Power to Rule Out a 50\% or 100\% Higher Adverse Event Rate in the Immediate Treatment Arm, Assuming Both Arms Have the Same Event Rate 83

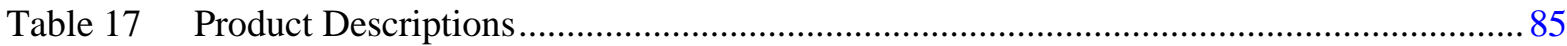


Product: MK-5172

Protocol/Amendment No.: 052-04

\section{LIST OF FIGURES}

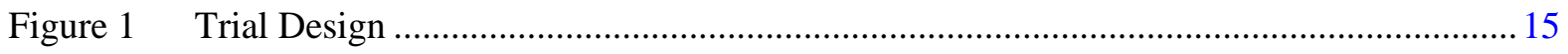




\section{SUMMARY OF CHANGES}

\section{PRIMARY REASON(S) FOR THIS AMENDMENT:}

\begin{tabular}{|c|c|c|}
\hline $\begin{array}{c}\text { Section } \\
\text { Number (s) }\end{array}$ & Section Title(s) & Description of Change (s) \\
\hline 5.1 .2 & $\begin{array}{ll}\text { Subject } & \text { Inclusion } \\
\text { Criteria } & \end{array}$ & Updated patient population in inclusion criteria \#3 and \#4 t \\
\hline
\end{tabular}

\section{ADDITIONAL CHANGE(S) FOR THIS AMENDMENT:}

\begin{tabular}{|c|c|c|}
\hline $\begin{array}{l}\text { Section } \\
\text { Number (s) }\end{array}$ & Section Title(s) & Description of Change (s) \\
\hline 2.1 & Trial Design & Updated study population \\
\hline 4.1 .3 & $\begin{array}{l}\text { Ongoing Clinical } \\
\text { Trials }\end{array}$ & Updated PN 035 Part B study cirrhotic results \\
\hline 4.2 .1 & $\begin{array}{l}\text { Rationale for The } \\
\text { Trial and Selected } \\
\text { Study Population }\end{array}$ & Updated study population \\
\hline 5.1 .2 & $\begin{array}{l}\text { Subject Inclusion } \\
\text { Criteria }\end{array}$ & Updated patient population in inclusion criteria \#3 and \#4 \\
\hline 5.1 .3 & $\begin{array}{l}\text { Subject Exclusion } \\
\text { Criteria }\end{array}$ & Updated laboratory exclusion values table for platelet criteria \\
\hline 7.2.3.2 & $\begin{array}{l}\text { Events of Clinical } \\
\text { Interest }\end{array}$ & $\begin{array}{l}\text { Added a caveat to definitions of lab ECIs for them to occur while on study therapy and not } \\
\text { associated with virological failure }\end{array}$ \\
\hline
\end{tabular}


Product: MK-5172

Protocol/Amendment No.: 052-04

\begin{tabular}{|l|l|l|}
\hline 8.2.4.1 & $\begin{array}{l}\text { Efficacy analysis } \\
\text { populations }\end{array}$ & Clarify the definition of analysis population and treatment group assignment \\
\hline 8.2 .8 & $\begin{array}{l}\text { Subgroup Analyses } \\
\text { and Effects of } \\
\text { Baseline Factors }\end{array}$ & The section was modified with respect to the changes of study inclusion criteria \\
\hline 8.2 .10 & $\begin{array}{l}\text { Compliance } \\
\text { (Medical } \\
\text { Adherence) }\end{array}$ & Added the section \\
\hline 11.0 & List of References & Added 1 new reference \\
\hline
\end{tabular}




\subsection{TRIAL SUMMARY}

\begin{tabular}{|c|c|}
\hline Abbreviated Title & $\begin{array}{l}\text { MK-5172 in Combination with MK-8742 in subjects with HCV and } \\
\text { Chronic Kidney Disease }\end{array}$ \\
\hline Trial Phase & Phase II/III \\
\hline Clinical Indication & Treatment of hepatitis C virus infection \\
\hline Trial Type & Interventional \\
\hline Type of control & Placebo \\
\hline Route of administration & Oral \\
\hline Trial Blinding & Double-blind \\
\hline Treatment Groups & $\begin{array}{l}\text { Immediate Treatment: MK-5172 } 100 \mathrm{mg}+\text { MK-8742 } 50 \mathrm{mg} \text { for } 12 \\
\text { weeks } \\
\text { Deferred Treatment: MK-5172 placebo+ MK-8742 placebo for } 12 \\
\text { weeks + 4 weeks unblinding period follow-up followed by MK-5172 } 100 \\
\text { mg + MK-8742 } 50 \mathrm{mg} \text { for } 12 \text { weeks } \\
\text { Intensive PK: MK-5172 } 100 \mathrm{mg}+\text { MK-8742 } 50 \mathrm{mg} \text { for } 12 \text { weeks (open- } \\
\text { label) }\end{array}$ \\
\hline Number of trial subjects & Approximately 220 subjects will be enrolled. \\
\hline \begin{tabular}{|l} 
Estimated duration of trial \\
\end{tabular} & $\begin{array}{l}\text { The sponsor estimates that the trial will require approximately } 3 \text { months } \\
\text { of enrollment ( } 12 \text { weeks) + up to } 60 \text { days of screening ( } 8.5 \text { weeks) + up } \\
\text { to } 24 \text { weeks of treatment (active or placebo/active) }+4 \text { week unblinding } \\
\text { period (deferred treatment arm only) }+24 \text { weeks of follow-up for a total } \\
\text { of } 72.5 \text { weeks from the time the first subject signs the informed consent } \\
\text { until the last subject's last visit. }\end{array}$ \\
\hline Duration of Participation & $\begin{array}{l}\text { Each subject will participate in the trial for approximately } 44.5 \text { or } 60.5 \\
\text { weeks (depending on the treatment arm) from the time the subject signs } \\
\text { the Informed Consent Form (ICF) through the final contact depending on } \\
\text { randomization. After a screening phase of } 60 \text { days, each subject will be } \\
\text { receiving assigned treatment for approximately } 12 \text { or } 24 \text { weeks. After } \\
\text { the end of treatment each subject will be followed for } 24 \text { weeks (deferred } \\
\text { treatment arm will be followed for an additional } 4 \text { weeks post-placebo } \\
\text { treatment). }\end{array}$ \\
\hline
\end{tabular}

A list of abbreviations used in this document can be found in Section 12.4.

\subsection{TRIAL DESIGN}

\subsection{Trial Design}

This is a randomized, parallel-group, multi-site, placebo controlled trial of MK-5172 and MK-8742 in subjects with Hepatitis C and Chronic Kidney disease (CKD) to be conducted in conformance with Good Clinical Practices.

The trial will enroll approximately 220 cirrhotic and non-cirrhotic, Genotype 1 (GT1), HCV patients who have chronic kidney disease (CKD). A definition of the HCV and CKD disease status for targeted subjects in this study is included in Table 1. Patients on maintenance 
Protocol/Amendment No.: 052-04

hemodialysis (including subjects awaiting renal transplant and subjects with a previous failed kidney transplant no longer on immunosuppressant therapy) and patients with CKD stages 45 who are not on hemodialysis will be enrolled with a minimum of $20 \%$ of patients in the latter category. Subjects must be either treatment naïve to all HCV treatments including any direct acting antivirals (DAA) or are intolerant or who have relapsed or were null-responders to a prior IFN-based treatment regimen. Subjects are required to undergo liver biopsy or non-invasive test to determine the presence or absence of cirrhosis.

Study subjects (210) will be randomized in a 1:1 ratio to receive MK-5172 $100 \mathrm{mg}$ QD and MK-8742 $50 \mathrm{mg}$ QD for 12 weeks with 24 weeks of follow-up after dosing is completed (immediate treatment group) or 12 weeks of placebo to MK-5172 and MK-8742 followed by unblinding (after a 4 week unblinding period) and then 12 weeks of MK-5172 $100 \mathrm{mg}$ QD and MK-8742 50 mg QD for 12 weeks with 24 weeks of follow-up after dosing is completed (deferred treatment group).

In addition, 10 subjects (5 on hemodialysis and 5 non-dialysis CKD) will be assigned to receive open-label MK-5172 $100 \mathrm{mg}$ QD and MK-8742 $50 \mathrm{mg}$ QD for 12 weeks with 24 weeks of follow-up after dosing is completed. These 10 subjects will constitute the Intensive PK arm. 


\section{Table 1 Definition of HCV and CKD in Target Subject Population}

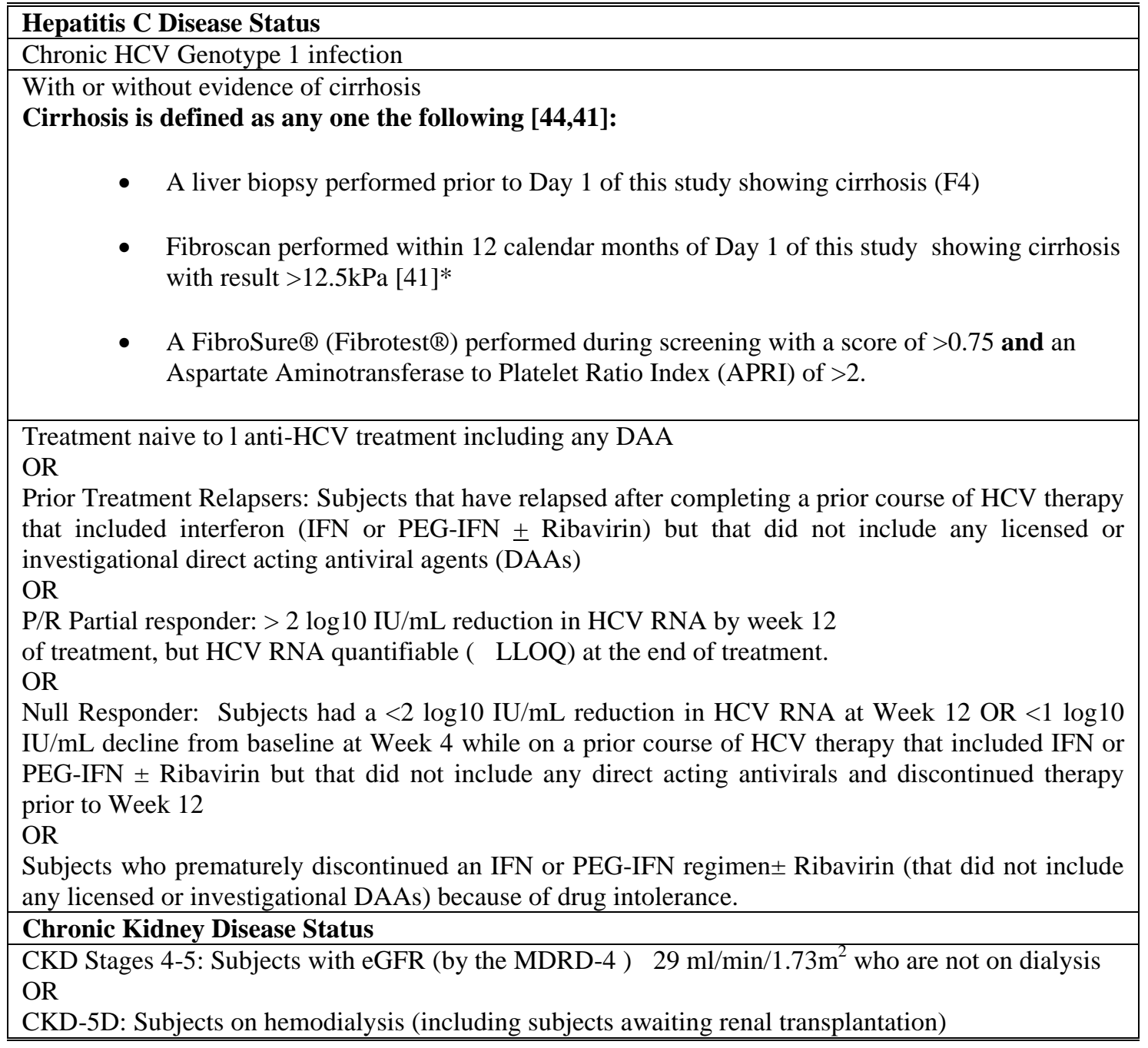

Administration of a 12 week regimen of MK-5172+MK-8742 regimen (without ribavirin) to treatment-naïve and treatment-experienced, cirrhotic and non-cirrhotic GT1 patients was highly efficacious in an ongoing PN035 trial (see Section 4.1.3).

Safety and tolerability will be carefully monitored throughout the study by the SPONSOR (or designee) in accordance with standard procedures and also by an external Data Monitoring Committee (eDMC).

Specific procedures to be performed during the trial, as well as their prescribed times and associated visit windows, are outlined in the Trial Flow Chart - Section 6.0. Details of each procedure are provided in Section 7.0 - Trial Procedures. 
Protocol/Amendment No.: 052-04

\subsection{Trial Diagram}

The trial design is depicted in Figure 1.

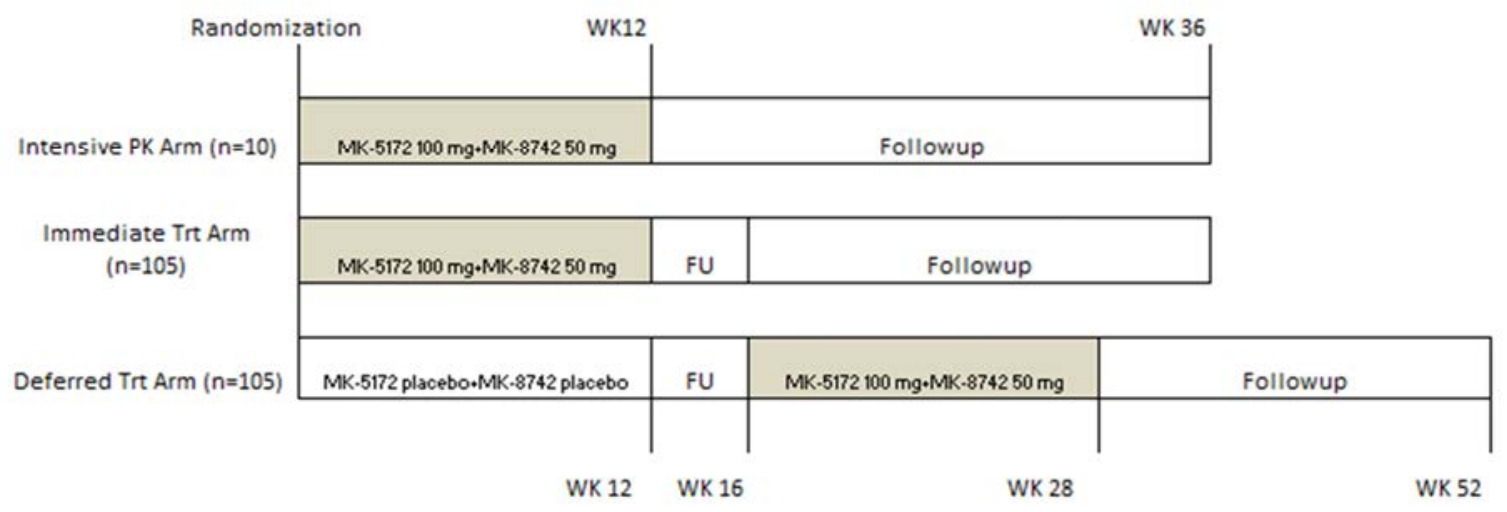

Figure 1 Trial Design

\subsection{OBJECTIVE(S) \& HYPOTHESIS(ES)}

\subsection{Primary Objective(s) \& Hypothesis(es)}

In subjects who have chronic kidney disease (CKD Stages 4-5) and chronic HCV GT1 infection with pre-treatment HCV RNA of at least 10,000 IU/mL:

The Primary Objective(s) are:

(1) Objective: To evaluate the efficacy of MK-5172 + MK-8742 in HCV GT1 subjects with chronic kidney disease (CKD) within the immediate treatment and the intensive PK groups.

Hypothesis: The proportion of HCV GT1 infected CKD 4-5 subjects achieving SVR (defined as HCV RNA <LLoQ (either TD(u) or TND) 12 weeks after the end of all study therapy will be superior to $45 \%$ (see Section 4.2.1- Rationale for Study).

(2) Objective: To evaluate the safety and tolerability of MK-5172 in combination with MK8742 in the immediate treatment group relative to the placebo treatment of the deferred treatment group. 


\subsection{Secondary Objective(s) \& Hypothesis(es)}

(1) Objective: To evaluate the efficacy of MK-5172 in combination with MK-8742 as assessed by the proportion of subjects achieving:

- $\mathrm{SVR}_{24}$ (Sustained Virologic Response 24 weeks after the end of all study therapy) within the immediate treatment and the intensive PK groups, defined as HCV RNA $<$ LLoQ (either TD(u) or TND) 24 weeks after the end of all study therapy.

- $\mathrm{SVR}_{4}$ (Sustained Virologic Response 4 weeks after the end of all study therapy), defined as HCV RNA <LLoQ (either TD(u) or TND) 4 weeks after the end of all study therapy.

- $\mathrm{SVR}_{12}$ (Sustained Virologic Response 12 weeks after the end of all study therapy), defined as HCV RNA <LLoQ (either TD(u) or TND) 12 weeks after the end of all study therapy on active period of deferred treatment arm,

- $\mathrm{SVR}_{12}$ (Sustained Virologic Response 12 weeks after the end of all study therapy), defined as HCV RNA <LLoQ (either TD(u) or TND) 12 weeks after the end of all study therapy for all active treatment arms combined.

(2) Objective: To evaluate the safety and tolerability of MK-5172 in combination with MK8742 for all treatment arms.

(3) Objective: To evaluate the emergence of viral resistance-associated variants (RAVs) resistant to MK-5172 and MK-8742 when administered as part of a combination regimen.

\subsection{Other Objectives (e.g., Tertiary, Exploratory, etc.)}

(1) Objective: To evaluate the pharmacokinetics (PK) of MK-5172 and MK-8742.

(2) Objective: To evaluate the pharmacokinetic/pharmacodynamics (PK/PD) relationship of MK-5172 and MK-8742 plasma levels to efficacy and safety.

(3) Objective: To evaluate biomarkers (e.g., proteins and metabolite production), that may be predictive of tolerability of study drugs and virologic response to MK-5172 in combination with MK-8742 by comparing biomarker levels over time in subjects who respond or fail study therapy.

(4) Objective: To describe and compare changes from baseline in health-related quality of life during and after active and placebo treatment periods.

(5) Objective: To assess the genetic variation in the human IL28B gene as a predictor of virologic response in each treatment arm.

(6) Objective: To determine the impact of HCV treatment on cryoglobulinemia in the patients with CKD. 
Product: MK-5172

Protocol/Amendment No.: 052-04

\subsection{BACKGROUND \& RATIONALE}

Redacted 
Product: MK-5172

Protocol/Amendment No.: 052-04

Redacted 
Product: MK-5172

Protocol/Amendment No.: 052-04

\section{Redacted}


Product: MK-5172

Protocol/Amendment No.: 052-04

Redacted 
Product: MK-5172

Protocol/Amendment No.: 052-04

\section{Redacted}


Product: MK-5172

Protocol/Amendment No.: 052-04

Redacted 
Product: MK-5172

Protocol/Amendment No.: 052-04

Redacted 
Product: MK-5172

Protocol/Amendment No.: 052-04

Redacted 
Product: MK-5172

Protocol/Amendment No.: 052-04

Redacted 
Product: MK-5172

Protocol/Amendment No.: 052-04

Redacted 
Product: MK-5172

Protocol/Amendment No.: 052-04

Redacted 
Product: MK-5172

Protocol/Amendment No.: 052-04

Redacted 


\subsection{METHODOLOGY}

\subsection{Entry Criteria}

\subsubsection{Diagnosis/Condition for Entry into the Trial}

Male/Female subjects with GT1 HCV who are cirrhotic or noncirrhotic and also have Chronic Kidney Disease who are at least 18 years of age will be enrolled in this trial.

\subsubsection{Subject Inclusion Criteria}

In order to be eligible for participation in this trial, the subject must:

1. Be $\geq 18$ years of age on day of signing informed consent.

2. Have documented chronic (at least 6 months) HCV GT1 infection (with no evidence of non typable or mixed genotypes) :

- Positive for anti-HCV antibody, HCV RNA, or an HCV genotype

- $\operatorname{HCV}$ RNA ( $\geq 10,000 \mathrm{IU} / \mathrm{mL}$ in peripheral blood)

3. Subjects with or without cirrhosis may be enrolled into this study. All subjects must have one of the below liver disease staging assessments as follows:

- Liver biopsy performed within 24 months of Day 1 (if subject is cirrhotic then there is no time restriction on biopsy) 
Protocol/Amendment No.: 052-04

- Fibroscan performed within 12 months of Day 1

- A FibroSure ${ }^{\circledR}$ (Fibrotest ${ }^{\circledR}$ ) and Aspartate Aminotransferase to Platelet Ratio Index (APRI) (APRI is automatically calculated by central laboratory) during Screening

In the absence of a definitive diagnosis of presence or absence of cirrhosis by the above critieria, a liver biopsy is required. Liver biopsy results supersede the results obtained by Fibroscan or FibroSure ${ }^{\circledR}$.

4. Have an HCV treatment status that is one of the following:

Treatment naïve: Naive to all anti-HCV treatment

Prior IFN or PEG-IFN \pm Ribavirin Treatment failures: Null responders, Partial responders, Relapsers

P/R Intolerant: Subjects were intolerant to a prior IFN or PEG-IFN \pm Ribavirin regimen, Subjects discontinued treatment prematurely and were therefore unable to complete a full course of therapy because of drug-related toxicity.

5. Have Chronic Kidney Disease defined as:

Subjects with GFR $\leq 29$ who are non-dialysis dependent (NDD) or have been on hemodialysis (HD) for at least 3 months (including subjects awaiting kidney transplant and subjects with failed kidney transplants no longer on immunosuppressant therapy).

6. Agree (if subject is of reproductive potential) to remain truly abstinent or use (or have their partner use) 2 acceptable methods of birth control from at least 2 weeks prior to Day 1 through 14 days after the last dose of study drugs, or longer if dictated by local regulations.

If acceptable by local regulatory agencies, methods of birth control allowed in the study are: intrauterine device (IUD), diaphragm with spermicide, hormonal contraceptives (e.g., birth control pills, transdermal patch, or injectables), contraceptive sponge, female condom, male condom with spermicide or vasectomy.

Note: Periodic abstinence (e.g., abstinence only on certain calendar days, abstinence only during ovulation period, use of symptothermal methods, use of post-ovulation methods and withdrawal) are not acceptable methods of contraception.

7. A female subject who is not of reproductive potential is eligible without requiring the use of contraception. A female subjects who is not of reproductive potentials is defined as one who has either 1 ) reached natural menopause (defined as 12 months with no menses without an alternative medical cause), 2) 6 weeks post surgical bilateral oophorectomy with or without hysterectomy, or 3) bilateral tubal ligation.

8. A male subject who is not of reproductive potential is eligible without requiring the use of contraception. A male subject who is not of reproductive potential is defined as: one who 
Protocol/Amendment No.: 052-04

has undergone a successful vasectomy. A successful vasectomy is defined as: (1) microscopic documentation of azoospermia, or (2) a vasectomy more than 2 years ago with no resultant pregnancy despite sexual activity post vasectomy.

9. understand the study procedures, alternative treatments available, risks involved with the study, and voluntarily agrees to participate by giving written informed consent.

10. The subject may also provide consent for Future Biomedical Research. However, the subject may participate in the main trial without participating in Future Biomedical Research

\subsubsection{Subject Exclusion Criteria}

The subject must be excluded from participating in the trial if the subject:

1. Is under the age of legal consent, is mentally or legally incapacitated, has significant emotional problems at the time of pre-study screening visit or expected during the conduct of the study or has a history of a clinically significant psychiatric disorder which, in the opinion of the investigator, would interfere with the study procedures.

2. Evidence of decompensated liver disease manifested by the presence of or history of ascites, gastric or variceal bleeding, hepatic encephalopathy or other signs or symptoms of advanced liver disease.

3. Is on peritoneal dialysis for management of Kidney disease

4. In the opinion of the investigator the subject has a high likelihood of receiving a renal transplant during the study treatment period (up to 24 weeks from Day 1).

5. Is coinfected with hepatitis B virus (e.g. HBsAg positive) or HIV.

6. Has a history of malignancy $\leq 5$ years prior to signing informed consent except for adequately treated basal cell or squamous cell skin cancer or in situ cervical cancer; or has evidence of hepatocellular carcinoma (HCC) or is under evaluation for other active or suspected malignancy.

7. Is taking or plans to take any of the prohibited medications listed in Section 5 of this protocol within 2 weeks of Day 1.

8. Is currently participating or has participated in a study with an investigational compound within 30 days of signing informed consent and is not willing to refrain from participating in another such study during the course of this study.

9. has a clinical diagnosis of substance abuse of the following specified drugs within specified timeframes: 
- alcohol, intravenous drugs, inhalational (not including marijuana), psychotropics, narcotics, cocaine use, prescription or over-the-counter drugs: within 1 year of the screening visit or, if shorter is judged by the investigator to be capable of complying with study procedures, OR

NOTE: Subjects receiving opiate agonist substitution therapy are not excluded from the study if, in the opinion of the investigator, the subject is capable of complying with all study procedures

- $\quad$ history of marijuana use is deemed excessive by a physician investigator or is interfering with the subject's daily function. If subject's marijuana use is not deemed excessive and does not interfere with daily function, subject must be instructed to discontinue any current use of recreational marijuana prior to entry into trial and throughout the trial period.

10. Female subject who is pregnant or breast-feeding, or expecting to conceive or donate eggs from Day 1 through 14 days after the last dose of study drugs, or longer if dictated by local regulations or male subject who is expecting to donate sperm from Day 1 through 14 days after the last dose of study drugs, or longer if dictated by local regulations.

11. Has any of the following conditions:

- Organ transplants (including hematopoietic stem cell transplants) other than kidney, cornea and hair.

- $\quad$ Poor venous access in non-dialysis patients that precludes routine peripheral blood sampling required for this trial.

- $\quad$ Subject with a history of gastric surgery (e.g., stapling, bypass) or subject with a history of malabsorption disorders (e.g., celiac sprue disease).

- Any medical condition requiring, or likely to require, chronic systemic administration of corticosteroids during the course of the trial.

- Has uncontrolled or poorly controlled hypertension including but not limited to hypertensive emergency or hospitalization for hypertension in preceding 3 months.

- Diagnosed with a significant cardiovascular disorder (e.g. MI or unstable angina) or has had a cardiovascular procedure (e.g. CABG or PTCA) within 3 months prior to signing informed consent.

- Has new or worsening signs or symptoms of congestive heart failure within 3 months of signing informed consent. 
Protocol/Amendment No.: 052-04

- Has severe active peripheral vascular disease, (e.g., manifested by claudication with minimal activity, a non-healing ischemic ulcer, or disease which is likely to require intervention such as with bypass or angioplasty).

- Has a recent (within 3 months prior to signing informed consent) diagnosis, episode or recurrence of stroke, TIA or neurological disorder, including but not limited to seizures, blackouts, or a recent (within 3 months prior to signing informed consent) change in the dose or class of medications used to treat these conditions.

12. Subject has any condition, prestudy laboratory abnormality or ECG abnormality, or history of any illness, which, in the opinion of the investigator, might confound the results of the study or pose additional risk in administering the study drugs to the subject.

13. Had a life-threatening SAE during the screening period.

14. Has evidence or history of chronic hepatitis not caused by HCV, including but not limited to nonalcoholic steatohepatitis (NASH), drug-induced hepatitis, and autoimmune hepatitis.

NOTE: Subjects with history of acute non-HCV-related hepatitis, which resolved $>6$ months before study entry, can be enrolled.

15. For subjects diagnosed with diabetes mellitus, chart documented HbA1c $>8.5 \%$ to exclude uncontrolled diabetics

16. Has exclusionary laboratory values as listed below (Table 4):

Note: If any of the laboratory exclusion criteria below are met, the site may have the abnormal value retested one time.

Table 4 Laboratory Exclusionary Values

\begin{tabular}{|c|l|}
\hline \hline Laboratory Assessment & \multicolumn{1}{|c|}{ Exclusionary Value } \\
\hline eGFR & $>29 \mathrm{~mL} / \mathrm{min}$ \\
\hline hemoglobin & $<9.0 \mathrm{~g} / \mathrm{dL}$ \\
\hline neutrophils & $<1.5 \times 10^{3} / \mu \mathrm{L}\left(<1.2 \times 10^{3} / \mu \mathrm{L}\right.$ for Blacks $)$ \\
\hline platelets & $<70 \times 10^{3} / \mu \mathrm{L}$ \\
\hline direct bilirubin & $>1.5 \times \mathrm{ULN}$ \\
\hline Total Bilirubin & $>1.6 \mathrm{mg} / \mathrm{dL}$ unless history of Gilbert's disease. (If Gilbert's disease is the proposed \\
& etiology, this must be documented in the subject's chart) \\
\hline Serum Albumin & $<3.0 \mathrm{~g} / \mathrm{dL}$ (lower limit of normal) of laboratory reference range \\
\hline INR & $>1.7$, unless subject has a stable INR on an anticoagulant regimen \\
\hline ALT & $>350$ \\
\hline AST & $>350$ \\
\hline \hline
\end{tabular}


17. Is or has an immediate family member (spouse or children) who is investigational site or sponsor staff directly involved with this trial.

\subsection{Trial Treatment(s)}

The treatment(s) to be used in this trial are outlined below in Table 5.

Table 5 Trial Treatment

\begin{tabular}{|c|c|c|c|c|c|c|}
\hline \hline Drug & Weight & Dose/Potency & $\begin{array}{c}\text { Dose } \\
\text { Frequency }\end{array}$ & $\begin{array}{c}\text { Route of } \\
\text { Administra- } \\
\text { tion }\end{array}$ & $\begin{array}{c}\text { Regimen/Trea } \\
\text { tment Period }\end{array}$ & Use \\
\hline MK-5172 & N/A & $100 \mathrm{mg}$ & QD & Oral & 12 Weeks & experimental \\
\hline MK-8742 & N/A & $50 \mathrm{mg}$ & QD & Oral & 12 Weeks & experimental \\
\hline MK-5172 Placebo & N/A & 0 & QD & Oral & 12 Weeks & experimental \\
\hline MK-8742 Placebo & N/A & 0 & QD & Oral & 12 Weeks & experimental \\
\hline \hline
\end{tabular}

The first dose of trial treatment will be taken by the subject in the evening of Day 1 (Visit 2) for all subjects (Immediate Treatment, Deferred Treatment and Intensive PK arms) and in the evening of the Week 16 visit (Visit 10) only for subjects in the deferred treatment arm. Subsequent dosing will be taken in the evenings by the subject at approximately the same time each day (except for Week 12 and Week 28 doses which must be withheld the night before for the predose PK collection at those visits).

The single entity tablet formulations will be used in the Intensive PK arm and for the first 12 weeks of dosing in the Immediate Treatment and Deferred Treatment arms. The fixed dose combination formulation will be used for Week 16 to Week 28 dosing in the Deferred Treatment arm.

The investigator shall take responsibility for and shall take all steps to maintain appropriate records and ensure appropriate supply, storage, handling, distribution and usage of trial treatments in accordance with the protocol and any applicable laws and regulations.

\subsubsection{Dose Selection/Modification}

\subsubsection{Dose Selection (Preparation)}

The rationale for selection of doses to be used in this trial is provided in Section 4.0 Background \& Rationale. There are no specific calculations or evaluations required to be performed in order to administer the proper dose to each subject.

\subsubsection{Dose Modification (Escalation/Titration/Other)}

Dose modification of MK-5172 and MK-8742 is not permitted. 


\subsubsection{Timing of Dose Administration}

Subjects will be instructed to take MK-5172 and MK-8742 together at bedtime. Phosphate binders should be taken at least 3 hours before or at least 3 hours after taking the investigational study medications.

If a subject misses a dose of MK-5172 and/or MK-8742 and it is less than 8 hours before the next dose, the missed dose should be skipped and the normal dosing schedule resumed. Subjects should not double the next dose in order to compensate for what has been missed.

For the Week 12 and 28 (for deferred treatment arm) visits, all subjects will withhold their last evening dose of study medications. Subjects will have a predose sample taken the next morning at their study visit, subjects will then take their study medications, and have a 2 hour post dose sample taken.

\subsubsection{Trial Blinding/Masking}

A double-blind/masking technique will be used. MK-5172 and MK-8742 and placebo will be packaged identically so that blind/masking is maintained. The subject, the investigator and Sponsor personnel or delegate(s) who are involved in the [treatment] or clinical evaluation of the subjects are unaware of the group assignments.

The subject, the investigator and the Sponsor will not know the treatment they are administered or the HCV RNA results through Week 12 of the study, including in-house team responsible for medical monitoring. A separate, in-house unblinded team will have access to the treatment group assignments and HCV RNA results. (Please note, the 10 subjects assigned to the intensive PK Arm will be open-label)

Pharmacokinetic (PK) measurements will be conducted in support of $\mathrm{PK}$ evaluations. Additionally, a small team as specified in a separate Modeling and Simulation (M\&S) Modeling Analysis Plan, and who are separate from the study team, will be unblinded for the purpose of preparing the pharmacokinetic analyses. No PK data or results from the PK analyses will be shared with the study team, and the unblinded group will not be members of the study team.

\section{$\underline{\text { Unblinding Procedures }}$}

A subject will not be unblinded until the Follow-Up Week 4 visit. All safety data through the first 12 weeks of treatment for that subject will be cleaned prior to unblinding, changes to the causality asessments that occurred through Week 12 will not be allowed after the subject is unblinded at Follow-Up Week 4.

See Section 7.1.5.2, Blinding/Unblinding, for a description of the method of unblinding a subject during the trial, should such an action be warranted. 


\subsection{Randomization or Treatment Allocation}

Treatment Allocation will occur centrally using an interactive voice response system (IVRS). In order to ensure enrollment of at least 20\% CKD 4-5 NDD subjects, the site will identify if the subject is HD or NDD at the time of screening. In addition the subject's prior HCV treatment status will be entered into the IVRS system at screening.

\subsection{Stratification}

Randomization will be stratified according to the following factors:

- Dialysis: Yes/No

- Diabetes: Yes/No

\subsection{Concomitant Medications/Vaccinations (Allowed \& Prohibited)}

Medications or vaccinations specifically prohibited in the exclusion criteria are not allowed during the dosing period. If there is a clinical indication for any medication or vaccination specifically prohibited during dosing period, discontinuation from trial therapy or vaccination may be required. The investigator should discuss any questions regarding this with the Sponsor Clinical Director. The final decision on any supportive therapy or vaccination rests with the investigator and/or the subject's primary physician. However, the decision to continue the subject on trial therapy or vaccination schedule requires the mutual agreement of the investigator, the Sponsor and the subject.

It is important for investigators to review each medication (prescription and non-prescription) the subject is taking before starting the study and at each study visit.

- At each visit, subjects should be questioned about any new drug they are taking.

- To minimize the risk of adverse drug interactions, every effort should be made to limit the number of concomitant drugs to those that are truly essential.

- Drugs known to be hepatotoxic (i.e., drugs with a warning of hepatotoxicity in the package insert) should be avoided during the dosing period. Investigators are encouraged to review each medication for potential hepatotoxicity by searching the www.livertox.nih.gov website.

The following medications/therapies are contraindicated during the dosing period:

Known hepatotoxic drugs, including but not limited to:

- Etofoxine

- Isoniazid

- Nitrofurantoin

- Phenytoin 
Protocol/Amendment No.: 052-04

\section{$\underline{\text { Herbal supplements }}$}

Strong CYP3A/P-gp inhibitors, including but not limited to:

- Antibiotics: clarithromycin, erythromycin, telithromycin

- Antifungals: itraconazole, ketoconazole, voriconazole

- Antihypertensives: nifedipine

- Nefazodone

$\underline{\text { Strong and moderate CYP3A/P-gp inducers, including but not limited to: }}$

- Anti-infectives: nafcillin, rifampin

- Anticonvulsants: carbamazepine, phenytoin, phenobarbital

- bosentan

- modafinil

- St. John's Wort

OATP inhibitors, including but not limited to:

- Immunosuppressants: cyclosporine

- Anti-infectives: rifampin

- Lipid lowering agents: gemfibrozil

- eltrombopag

- lapatinib

HIV medications, including but not limited to:

- efavirenz

- etravirine

- all ritonavir-boosted and unboosted HIV protease inhibitors

HMG-CoA reductase inhibitors (statins), including but not limited to:

- simvastatin

- fluvastatin

- rosuvastatin

- atorvastatin

- pitavastatin

- pravastatin at doses greater than $10 \mathrm{mg}$

- Note: Questions regarding use of other statins should be directed to the Sponsor.

In general, CYP3A4 substrates with narrow therapeutic ranges (e.g. alfentanil, astemizole, cisapride, dihydroergotamine, ergotamine, fentanyl, pimozide, quinidine, terfenadine) are not 
prohibited, but their levels have the potential to be increased by approximately $30 \%$. Therefore, subjects taking these medications should be monitored closely or dose adjusted appropriately.

Investigational agents are not permitted.

Systemic corticosteroids (dose equivalent to $\geq 10 \mathrm{mg}$ prednisone per day, except in the case of rapid steroid tapers $<1$ week in duration) are not permitted.

Concomitant medications and therapies discontinued during the dosing period may be restarted 2 weeks after the last dose of study drug is administered and may be continued during the follow-up period.

\section{Allowed Medications}

The following concomitant medications are allowed in this study:

Phosphate binders such as

- calcium carbonate/calcium acetate

- sevelamer

- lanthanum

Note: Subjects taking phosphate binders must do so either at least 3 hours before or at least 3 hours after taking the investigational study medications.

$\underline{\text { Statins such as }}$

- pravastatin: use the lowest possible effective dose, but do not exceed a daily dose of $10 \mathrm{mg}$

Note: Questions regarding use of other statins should be directed to the Sponsor.

Medications for anemia such as

- $\quad$ Erythropoetin

Antihypertensives

- ACE inhibitors/ARBs: enalapril, captopril, lisinopril, ramipril, valsartan, losartan, telmisartan

- Most beta blockers: atenolol, metoprolol, propranolol

Note: for other beta blockers, please consult with the Sponsor

- calcium-channel blockers: verapamil, diltiazem, amlodipine

Note: For other antihypertensives, please consult with the Sponsor

- hydralazine, clonidine, minoxidil, isosorbide nitrates

Medications for hyperparathyroidism

- ergocalciferol

- vitamin D analogs: calcitriol, paricalcitol, doxercalciferol, alfacalcidol, falecalcitriol, 22-oxacalcitriol

- calcimimetics: cinacalcet 
Diuretics

- HCTZ

- furosemide

- spironolactone

- triamterene

Hypoglycemic agents

- Insulin

- Sitagliptin

$\underline{\text { Anticoagulants }}$

- Warfarin

Note: For other medications not listed here, please consult with the Sponsor.

\subsection{Rescue Medications \& Supportive Care}

No rescue or supportive medications are specified to be used in this trial.

\subsection{Diet/Activity/Other Considerations}

\section{Dietary Considerations}

MK-5172 and MK-8742 can be taken without regard to food; however, intake of grapefruit or grapefruit juice is prohibited during the dosing period of the trial.

Subjects taking phosphate binders must do so either 3 hours before or three hours after the investigational study medications administered in this study.

\section{Considerations for Study Visits}

Procedures visits should be scheduled as close to the indicated study days and study weeks as possible. See the Study Flow Chart in Section 6 for a complete listing of study procedures required at each visit. Collection of PK samples (predose and/or postdose) must be taken as indicated in Table 7, Table 8, and Table 9 in Section 7.1.4.2

\subsection{Subject Withdrawal/Discontinuation Criteria}

Subjects may withdraw consent at any time for any reason or be dropped from the trial at the discretion of the investigator should any untoward effect occur. In addition, a subject may be withdrawn by the investigator or the Sponsor if enrollment into the trial is inappropriate, the trial plan is violated, or for administrative and/or other safety reasons. Specific details regarding discontinuation or withdrawal procedures; including specific details regarding withdrawal from Future Biomedical Research, are provided in Section 7.1.4 - Other Procedures. 
In this trial, a subject may discontinue from treatment but continue to participate in the regularly scheduled activities, as long as the subject does not withdraw consent. Discontinuation from treatment is permanent. Once a subject has discontinued treatment, even though he/she continues to be monitored in the trial, he/she shall not be allowed to begin treatment again.

A subject must be discontinued from the trial for any of the following reasons:

- The subject or legal representative (such as a parent or legal guardian) withdraws consent.

- The subject has a medical condition or personal circumstance which, in the opinion of the investigator and/or Sponsor, places the subject at unnecessary risk through continued participation in the trial or does not allow the subject to adhere to the requirements of the protocol.

A subject must be discontinued from treatment (but may continue to be monitored in the trial) for any of the following reasons:

- $\quad$ Subject meets any virologic failure criteria (see Section 4.2.3.1.1.2)

- Subject becomes pregnant during the trial.

- A physician investigator feels it is in best interest of the subject to discontinue.

- Subject receives a renal transplant.

- $\quad$ The subject's ALT or AST increases to >500 IU/L.

- The subject's ALT or AST increases to $>3 \mathrm{x}$ baseline, is $>100 \mathrm{IU} / \mathrm{L}$, and there is a simultaneous increase in total bilirubin $>2 x$ ULN and/or INR $>1.5$.

- The subject's ALT or AST increases to $>3 x$ the nadir value, is $>100 \mathrm{IU} / \mathrm{L}$, and there is a simultaneous increase in total bilirubin $>2 x$ ULN and/or INR $>1.5$.

- The subject's ALT or AST increases to $>3 x$ baseline, is $>100 \mathrm{IU} / \mathrm{L}$, and is temporally associated with the new onset or worsening of any of the following adverse events that are of moderate or severe intensity and deemed by the investigator to be at least possibly related to MK-5172 and or MK-8742: nausea, vomiting, right upper quadrant pain or tenderness, and/or eosinophilia (>5\%).

- The subject's ALT or AST increases to $>3 x$ the nadir value, is $>100 \mathrm{IU} / \mathrm{L}$, and is temporally associated with the new onset or worsening of any of the following adverse events that are of moderate or severe intensity and deemed by the investigator to be at least possibly related to MK-5172 and or MK-8742: nausea, vomiting, right upper quadrant pain or tenderness, and/or eosinophilia (>5\%). 
- The subject's alkaline phosphatase increases to $>3 x$ ULN, there is a simultaneous increase in total bilirubin $>2 x$ ULN and other causes of elevated alkaline phosphatase are excluded.

- The subject's alkaline phosphatase increases to $>5 x$ ULN and other causes of elevated alkaline phosphatase are excluded.

A subject may be discontinued from treatment for any of the following reasons:

- $\mathrm{SAE}$ assessed by the physician investigator as possibly or probably related to study medication. Investigator may continue the subject in the trial, if it is deemed to be in the best interest of the subject to stay on the study treatment.

- Failure to comply with the dosing, evaluations, or other requirements of the trial

\subsection{Subject Replacement Strategy}

A subject who discontinues from the trial will not be replaced.

\subsection{Beginning and End of the Trial}

The overall trial begins when the first subject signs the informed consent form. The overall trial ends when the last subject completes the last trial visit, discontinues from the trial or is lost to follow-up (i.e. the subject is unable to be contacted by the investigator).

\subsection{Clinical Criteria for Early Trial Termination}

Early trial termination will be the result of the criteria specified below:

\section{Early Trial Termination Due to Safety}

If $>10$ of 115 in the immediate treatment arm or Intensive PK arm meet any of the safety criteria listed below, then the study should be terminated.

- ALT or AST increases to >500 IU/L.

- ALT or AST increases to $>3 x$ baseline, is $>100 \mathrm{IU} / \mathrm{L}$, and there is a simultaneous increase in total bilirubin $>2 \mathrm{x}$ ULN and/or INR $>1.5$.

- ALT or AST increases to $>3 x$ the nadir value, is $>100 \mathrm{IU} / \mathrm{L}$, and there is a simultaneous increase in total bilirubin $>2 x$ ULN and/or INR $>1.5$.

- ALT or AST increases to $>3 x$ baseline, is $>100 \mathrm{IU} / \mathrm{L}$, and is temporally associated with the new onset or worsening of any of the following adverse events that are of moderate or severe intensity and deemed by the investigator to be at least possibly related to MK-5172: nausea, vomiting, right upper quadrant pain or tenderness, and/or eosinophilia (>5\%). 
Protocol/Amendment No.: 052-04

- ALT or AST increases to $>3 x$ the nadir value, is $>100 \mathrm{IU} / \mathrm{L}$, and is temporally associated with the new onset or worsening of any of the following adverse events that are of moderate or severe intensity and deemed by the investigator to be at least possibly related to MK-5172: nausea, vomiting, right upper quadrant pain or tenderness, and/or eosinophilia (>5\%).

- alkaline phosphatase increases to $>3 x$ ULN, a simultaneous increase in total bilirubin $>2 \mathrm{x}$ ULN and other causes of elevated alkaline phosphatase are excluded.

- alkaline phosphatase increases to >5x ULN and other causes of elevated alkaline phosphatase are excluded.

\section{Early Trial Termination Due to Virologic Failure Criteria (rebound, non-response, breakthrough, relapse):}

All Early Trial Termination decisions will be based on the per-protocol population.

If $>7$ of the first 20 patients in the immediate treatment group meet virologic failure criteria, no additional subjects will be enrolled and the study will be terminated. 
Protocol/Amendment No.: 052-04

\subsection{TRIAL FLOW CHART}

\begin{tabular}{|c|c|c|c|c|c|c|c|c|c|c|c|c|c|c|c|c|c|c|c|c|c|c|c|c|}
\hline & & & & \multicolumn{16}{|c|}{ Treatment Days/Weeks } & \multicolumn{3}{|c|}{ Follow-Up Weeks } & \multicolumn{2}{|c|}{ Unscheduled Visits } \\
\hline Immediate Treatment Arm & \multirow{3}{*}{ Screen } & \multirow{3}{*}{$\begin{array}{l}\overrightarrow{\vec{a}} \\
\vec{\Xi}\end{array}$} & \multirow{3}{*}{$\hat{\vec{\Xi}}$} & \multirow{3}{*}{2} & \multirow{3}{*}{3} & \multirow{3}{*}{4} & \multirow{3}{*}{6} & \multirow{3}{*}{8} & \multirow{3}{*}{10} & \multirow{3}{*}{12} & \multirow{2}{*}{ NA } & \multirow{2}{*}{ NA } & \multirow{2}{*}{ NA } & \multirow{2}{*}{ NA } & & & & & & & & & & \\
\hline Intensive PK Arm & & & & & & & & & & & & & & & NA & NA & NA & NA & \multirow{2}{*}{\begin{tabular}{|l|} 
NA \\
28 \\
\end{tabular}} & \multirow[t]{2}{*}{ FU4 } & \multirow[t]{2}{*}{ FU 12} & \multirow[t]{2}{*}{ FU 24} & Viral Fail Conf & Discon \\
\hline Deferred Treatment Arm & & & & & & & & & & & 16 & 17 & 18 & 19 & 20 & 22 & 24 & 26 & & & & & & \\
\hline Visit No. & 1 & 2 & 3 & 4 & 5 & 6 & 7 & 8 & 9 & 10 & 11 & 12 & 13 & 14 & 15 & 16 & 17 & 18 & 19 & 20 & 21 & 22 & 23 & 24 \\
\hline Visit Window & -60 days & NA & \begin{tabular}{|l}
$-6 /+7$ \\
days
\end{tabular} & $\pm 1 \mathrm{w}$ & jeek & \begin{tabular}{|l|}
$-1 /+2$ \\
week \\
\end{tabular} & & $\pm 2 \mathrm{w}$ & & \begin{tabular}{|c|}
$-2 /+4$ \\
week \\
\end{tabular} & $\begin{array}{c} \pm 2 \\
\text { week }\end{array}$ & & $\pm 1 \mathrm{we}$ & & $\begin{array}{l}-1 /+2 \\
\text { week }\end{array}$ & & \pm 2 wee & & \begin{tabular}{|c|}
$-2 /+4$ \\
week \\
\end{tabular} & \begin{tabular}{|c|} 
\pm 2 \\
weeks
\end{tabular} & $\pm 4 \mathrm{w}$ & veeks & NA & \\
\hline ADMINISTRATIVE PROCE & DURES & & & & & & & & & & & & & & & & & & & & & & & \\
\hline Informed Consent & $\mathrm{x}$ & & & & & & & & & & & & & & & & & & & & & & & \\
\hline $\begin{array}{l}\text { Informed Consent for Future } \\
\text { Biomedical Research }\end{array}$ & $\mathrm{x}$ & & & & & & & & & & & & & & & & & & & & & & & \\
\hline Inclusion/Exclusion Criteria & $\mathrm{x}$ & & & & & & & & & & & & & & & & & & & & & & & \\
\hline Subject Identification Card & $\mathrm{x}$ & & & & & & & & & & & & & & & & & & & & & & & \\
\hline Medical History & $\mathrm{x}$ & & & & & & & & & & & & & & & & & & & & & & & \\
\hline Prior and Con-med Review & $\mathrm{x}$ & $\mathrm{x}$ & $\mathrm{x}$ & $\mathrm{x}$ & $\mathrm{x}$ & $\mathrm{x}$ & $\mathrm{x}$ & $\mathrm{x}$ & $\mathrm{x}$ & $\mathrm{x}$ & $\mathrm{x}$ & $\mathrm{x}$ & $\mathrm{x}$ & $\mathrm{x}$ & $\mathrm{x}$ & $\mathrm{x}$ & $\mathrm{x}$ & $\mathrm{x}$ & $\mathrm{x}$ & & & & $\mathrm{x}$ & $\mathrm{x}$ \\
\hline $\begin{array}{l}\text { Treatment } \\
\text { Allocation/Randomization }\end{array}$ & & $\mathrm{x}$ & & & & & & & & & & & & & & & & & & & & & & \\
\hline $\begin{array}{l}\text { Review Study Medication } \\
\text { Diary }\end{array}$ & & $\mathrm{x}$ & $\mathrm{x}$ & $\mathrm{x}$ & $\mathrm{x}$ & $\mathrm{x}$ & $\mathrm{x}$ & $\mathrm{x}$ & $\mathrm{x}$ & $\mathrm{x}$ & $\mathrm{x}$ & $\mathrm{x}$ & $\mathrm{x}$ & $\mathrm{x}$ & $\mathrm{x}$ & $\mathrm{x}$ & $\mathrm{x}$ & $\mathrm{x}$ & $\mathrm{x}$ & & & & $\mathrm{x}$ & $\mathrm{x}$ \\
\hline $\begin{array}{l}\text { Unblinding of treatment } \\
\text { assignment }\end{array}$ & & & & & & & & & & & $\mathrm{X}^{14}$ & & & & & & & & & $\mathrm{X}^{14}$ & & & & \\
\hline CLINICAL SAFETY EVALU & JATION & & & & & & & & & & & & & & & & & & & & & & & \\
\hline Physical Examination $^{1}$ & $\mathrm{x}$ & $\mathrm{x}$ & & & & $\mathrm{x}$ & & & & $\mathrm{x}$ & $\mathrm{x}$ & & & & $\mathrm{x}$ & & & & & $\mathrm{x}$ & & & & $\mathrm{x}$ \\
\hline Weight & $\mathrm{x}$ & $\mathrm{x}$ & & & & & & & & $\mathrm{x}$ & $\mathrm{x}$ & & & & & & & & & $\mathrm{x}$ & & & & $\mathrm{x}$ \\
\hline Height & $\mathrm{x}$ & & & & & & & & & & & & & & & & & & & & & & & \\
\hline 12-Lead ECG & $\mathrm{x}$ & & & & & $\mathrm{x}$ & & & & $\mathrm{x}$ & $\mathrm{x}$ & & & & $\mathrm{x}$ & & & & $\mathrm{x}$ & $\mathrm{x}$ & & & & $\mathrm{x}$ \\
\hline Vital Signs & $\mathrm{x}$ & $\mathrm{x}$ & $\mathrm{x}$ & $\mathrm{x}$ & $\mathrm{x}$ & $\mathrm{x}$ & $\mathrm{x}$ & $\mathrm{x}$ & $\mathrm{x}$ & $\mathrm{x}$ & $\mathrm{x}$ & $\mathrm{x}$ & $\mathrm{x}$ & $\mathrm{x}$ & $\mathrm{x}$ & $\mathrm{x}$ & $\mathrm{x}$ & $\mathrm{x}$ & $\mathrm{x}$ & $\mathrm{x}$ & & & $\mathrm{x}$ & $\mathrm{x}$ \\
\hline $\begin{array}{l}\text { Subject confirmation of birth } \\
\text { control }\end{array}$ & $\mathrm{x}$ & $\mathrm{x}$ & $\mathrm{x}$ & $\mathrm{x}$ & $\mathrm{x}$ & $\mathrm{x}$ & $\mathrm{x}$ & $\mathrm{x}$ & $\mathrm{x}$ & $\mathrm{x}$ & $\mathrm{x}$ & $\mathrm{x}$ & $\mathrm{x}$ & $\mathrm{x}$ & $\mathrm{x}$ & $\mathrm{x}$ & $\mathrm{x}$ & $\mathrm{x}$ & $\mathrm{x}$ & $\mathrm{x}^{16}$ & & & $\mathrm{x}$ & $\mathrm{x}$ \\
\hline $\begin{array}{l}\text { Review (Serious) Adverse } \\
\text { Events }^{2}\end{array}$ & $\mathrm{x}$ & $\mathrm{x}$ & $\mathrm{x}$ & $\mathrm{x}$ & $\mathrm{x}$ & $\mathrm{x}$ & $\mathrm{x}$ & $\mathrm{x}$ & $\mathrm{x}$ & $\mathrm{x}$ & $\mathrm{x}$ & $\mathrm{x}$ & $\mathrm{x}$ & X & $\mathrm{x}$ & $\mathrm{x}$ & $\mathrm{x}$ & $\mathrm{x}$ & $\mathrm{x}$ & $\mathrm{x}$ & $\mathrm{x}$ & $\mathrm{x}$ & $\mathrm{x}$ & $\mathrm{x}$ \\
\hline
\end{tabular}


Product: MK-5172

Protocol/Amendment No.: 052-04

\begin{tabular}{|c|c|c|c|c|c|c|c|c|c|c|c|c|c|c|c|c|c|c|c|c|c|c|c|c|}
\hline & & & & \multicolumn{16}{|c|}{ Treatment Days/Weeks } & \multicolumn{3}{|c|}{ Follow-Up Weeks } & \multicolumn{2}{|c|}{ Unscheduled Visits } \\
\hline Immediate Treatment Arm & \multirow{3}{*}{ Screen } & \multirow{3}{*}{ 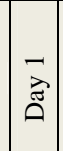 } & \multirow{3}{*}{$\hat{\vec{\Xi}}$} & \multirow{3}{*}{2} & \multirow{3}{*}{3} & \multirow{3}{*}{4} & \multirow{3}{*}{6} & \multirow{3}{*}{8} & \multirow{3}{*}{10} & \multirow{3}{*}{12} & \multirow[b]{2}{*}{ NA } & \multirow{2}{*}{ NA } & \multirow{2}{*}{ NA } & \multirow{2}{*}{ NA } & \multirow{2}{*}{ NA } & \multirow{2}{*}{ NA } & \multirow{2}{*}{ NA } & \multirow{2}{*}{ NA } & \multirow{2}{*}{ NA } & & & & & \\
\hline Intensive PK Arm* & & & & & & & & & & & & & & & & & & & & FU4 & FU 12 & FU 24 & Viral Fail Conf & Discon \\
\hline Deferred Treatment Arm & & & & & & & & & & & 16 & 17 & 18 & 19 & 20 & 22 & 24 & 26 & 28 & & & & & \\
\hline Visit No. & 1 & 2 & 3 & 4 & 5 & 6 & 7 & 8 & 9 & 10 & 11 & 12 & 13 & 14 & 15 & 16 & 17 & 18 & 19 & 20 & 21 & 22 & 23 & 24 \\
\hline Visit Window & -60 days & NA & \begin{tabular}{|l}
$-6 /+7$ \\
days
\end{tabular} & $\pm 1 \mathrm{w}$ & jeek & \begin{tabular}{|l|}
$-1 /+2$ \\
week
\end{tabular} & & $\pm 2 \mathrm{w}$ & & \begin{tabular}{|c|}
$-2 /+4$ \\
week \\
\end{tabular} & \begin{tabular}{|c|} 
\pm 2 \\
week
\end{tabular} & & $\pm 1 \mathrm{we}$ & & $\begin{array}{c}-1 /+2 \\
\text { week }\end{array}$ & & \pm 2 week & & $\begin{array}{l}-2 /+4 \\
\text { week }\end{array}$ & $\begin{array}{c} \pm 2 \\
\text { weeks }\end{array}$ & $\pm 4 \mathrm{w}$ & veeks & NA & \\
\hline LABORATORY SAFETY EV & VALUAT & IION & & & & & & & & & & & & & & & & & & & & & & \\
\hline Coagulation & $\mathrm{x}$ & $\mathrm{x}$ & $\mathrm{x}$ & $\mathrm{x}$ & $\mathrm{x}$ & $\mathrm{x}$ & $\mathrm{x}$ & $\mathrm{x}$ & $\mathrm{x}$ & $\mathrm{x}$ & $\mathrm{x}$ & $\mathrm{x}$ & $\mathrm{x}$ & $\mathrm{x}$ & $\mathrm{x}$ & $\mathrm{x}$ & $\mathrm{x}$ & $\mathrm{x}$ & $\mathrm{x}$ & $\mathrm{x}$ & $\mathrm{x}$ & $\mathrm{x}$ & $\mathrm{x}$ & $\mathrm{x}$ \\
\hline Chemistry \& Hematology & $\mathrm{x}$ & $\mathrm{x}$ & $\mathrm{x}$ & $\mathrm{x}$ & $\mathrm{x}$ & $\mathrm{x}$ & $\mathrm{x}$ & $\mathrm{x}$ & $\mathrm{x}$ & $\mathrm{x}$ & $\mathrm{x}$ & $\mathrm{x}$ & $\mathrm{x}$ & $\mathrm{x}$ & $\mathrm{x}$ & $\mathrm{x}$ & $\mathrm{x}$ & $\mathrm{x}$ & $\mathrm{x}$ & $\mathrm{x}$ & $\mathrm{x}$ & $\mathrm{x}$ & $\mathrm{x}$ & $\mathrm{x}$ \\
\hline Urinalysis $^{3}$ & $\mathrm{x}$ & & $\mathrm{x}$ & $\mathrm{x}$ & $\mathrm{x}$ & $\mathrm{x}$ & $\mathrm{x}$ & $\mathrm{x}$ & $\mathrm{x}$ & $\mathrm{x}$ & & $\mathrm{x}$ & $\mathrm{x}$ & $\mathrm{x}$ & $\mathrm{x}$ & $\mathrm{x}$ & $\mathrm{x}$ & $\mathrm{x}$ & $\mathrm{x}$ & $\mathrm{x}$ & $\mathrm{x}$ & $\mathrm{x}$ & $\mathrm{x}$ & $\mathrm{x}$ \\
\hline Cryoglobulinemia labs $^{15}$ & & $\mathrm{x}$ & & & & & & & & $x$ & & & & & & & & & $x$ & & $x$ & & & \\
\hline $\begin{array}{l}\text { HBA1C (only for subjects with } \\
\text { a prior diagnosis of diabetes) }\end{array}$ & $\mathrm{x}$ & & & & & & $\mathrm{x}$ & & & $\mathrm{x}$ & & & & & & $\mathrm{x}$ & & & $\mathrm{x}$ & & $\mathrm{x}$ & & & \\
\hline HBsAg & $\mathrm{x}$ & & & & & & & & & & & & & & & & & & & & & & & \\
\hline HIV screen & $\mathrm{x}$ & & & & & & & & & & & & & & & & & & & & & & & \\
\hline $\begin{array}{l}\text { Pregnancy Test (females of } \\
\text { child bearing potential only) }\end{array}$ & $\mathrm{x}$ & $\mathrm{x}$ & & & & & & & & $\mathrm{x}$ & & & & & & & & & $\mathrm{x}$ & & $\mathrm{x}$ & $\mathrm{x}$ & $\mathrm{x}$ & $\mathrm{x}$ \\
\hline PATIENT REPORTED OUT & ГCOME & & & & & & & & & & & & & & & & & & & & & & & \\
\hline SF36® Health Survey & & $\mathrm{x}$ & & & & & & & & $\mathrm{x}$ & & & & & & & & & $\mathrm{x}$ & & $\mathrm{X}$ & & & $\mathrm{x}$ \\
\hline PHARMACOKINETICS & & & & & & & & & & & & & & & & & & & & & & & & \\
\hline $\begin{array}{l}\text { MK-5172 (population PK in } \\
\text { all subjects) }\end{array}$ & & $\mathrm{X}$ & & & & & & & & $\mathrm{X}$ & & & & & & & & & $\mathrm{X}$ & & & & $\mathrm{x}$ & $\mathrm{x}$ \\
\hline $\begin{array}{l}\text { MK-8742 (population PK in } \\
\text { all subjects) }\end{array}$ & & $\mathrm{X}$ & & & & & & & & $\mathrm{X}$ & & & & & & & & & $\mathrm{X}$ & & & & $\mathrm{x}$ & $\mathrm{x}$ \\
\hline $\begin{array}{l}\text { MK-5172 (intensive PK Arm } \\
\text { only) }\end{array}$ & & $\mathrm{X}$ & & & & $\mathrm{X}$ & & & & $\mathrm{X}$ & & & & & & & & & & & & & $\mathrm{x}$ & $\mathrm{x}$ \\
\hline $\begin{array}{l}\text { MK-8742 (intensive PK Arm } \\
\text { only) }\end{array}$ & & $\mathrm{X}$ & & & & $\mathrm{X}$ & & & & $\mathrm{X}$ & & & & & & & & & & & & & $\mathrm{x}$ & $\mathrm{x}$ \\
\hline $\begin{array}{l}\text { MK-5172 (evening pre-dose } \\
\text { PK cohort only) }\end{array}$ & & & & & & $\mathrm{X}$ & & & & $\mathrm{X}$ & & & & & & & & & & & & & $\mathrm{x}$ & $\mathrm{x}$ \\
\hline $\begin{array}{l}\text { MK-8742 (evening pre-dose } \\
\text { PK cohort only) }\end{array}$ & & & & & & $\mathrm{X}$ & & & & $\mathrm{X}$ & & & & & & & & & & & & & $\mathrm{x}$ & $\mathrm{x}$ \\
\hline HCV EVALUATIONS & & & & & & & & & & & & & & & & & & & & & & & & \\
\hline HCV Genotype Determination & $\mathrm{x}$ & & & & & & & & & & $\mathrm{x}$ & & & & & & & & & & & & & \\
\hline HCV RNA Level $^{7}$ & $\mathrm{x}$ & $\mathrm{x}$ & $\mathrm{x}$ & $\mathrm{x}$ & $\mathrm{x}$ & $\mathrm{x}$ & $\mathrm{x}$ & $\mathrm{x}$ & $\mathrm{x}$ & $\mathrm{x}$ & $\mathrm{x}$ & $\mathrm{x}$ & $\mathrm{x}$ & $\mathrm{x}$ & $\mathrm{x}$ & $\mathrm{x}$ & $\mathrm{x}$ & $\mathrm{x}$ & $\mathrm{x}$ & $\mathrm{x}$ & $\mathrm{x}$ & $\mathrm{x}$ & $\mathrm{x}$ & $\mathrm{X}^{11}$ \\
\hline $\begin{array}{l}\text { Plasma for HCV Viral } \\
\text { Resistance and Biomarker }{ }^{5,7}\end{array}$ & & $\mathrm{x}$ & & & & & & & & & & & & & & & & & & $\mathrm{x}$ & $\mathrm{x}$ & $\mathrm{x}$ & $\mathrm{x}$ & $\mathrm{X}^{11}$ \\
\hline $\begin{array}{l}\text { Blood (DNA) for genetic } \\
\text { analysis }^{6}\end{array}$ & & $\mathrm{x}$ & & & & & & & & & & & & & & & & & & & & & & \\
\hline $\begin{array}{l}\text { Blood (DNA) for Future } \\
\text { Biomedical Research }^{7}\end{array}$ & & $\mathrm{x}$ & & & & & & & & & & & & & & & & & & & & & & \\
\hline
\end{tabular}




\begin{tabular}{|c|c|c|c|c|c|c|c|c|c|c|c|c|c|c|c|c|c|c|c|c|c|c|c|c|}
\hline & & & & \multicolumn{16}{|c|}{ Treatment Days/Weeks } & \multicolumn{3}{|c|}{ Follow-Up Weeks } & \multicolumn{2}{|c|}{ Unscheduled Visits } \\
\hline Immediate Treatment Arm & \multirow{3}{*}{ Screen } & \multirow{3}{*}{$\mid \begin{array}{l}\overrightarrow{3} \\
\overrightarrow{\widetilde{a}}\end{array}$} & \multirow{3}{*}{$\hat{\overparen{\oplus}}$} & \multirow{3}{*}{2} & \multirow{3}{*}{3} & \multirow{3}{*}{4} & \multirow{3}{*}{6} & \multirow{3}{*}{8} & \multirow{3}{*}{10} & \multirow{3}{*}{12} & \multirow{3}{*}{$\mathrm{N}$} & \multirow{3}{*}{\begin{tabular}{|l|} 
NA \\
17 \\
\end{tabular}} & \multirow{3}{*}{\begin{tabular}{|l|} 
NA \\
18 \\
\end{tabular}} & \multirow{3}{*}{$\begin{array}{l}\text { NA } \\
19 \\
\end{array}$} & \multirow{3}{*}{\begin{tabular}{l|} 
NA \\
20 \\
\end{tabular}} & \multirow{3}{*}{\begin{tabular}{|l|} 
NA \\
22 \\
\end{tabular}} & \multirow{3}{*}{\begin{tabular}{|l|} 
NA \\
24 \\
\end{tabular}} & \multirow{3}{*}{\begin{tabular}{|l|} 
NA \\
26 \\
\end{tabular}} & \multirow{3}{*}{\begin{tabular}{|l|} 
NA \\
28
\end{tabular}} & \multirow{3}{*}{ FU4 } & \multirow{3}{*}{ FU 12} & \multirow{3}{*}{ FU 24} & \multirow{3}{*}{\begin{tabular}{||} 
Unsched/HCV \\
Viral Fail Conf \\
Visit
\end{tabular}} & \multirow{3}{*}{$\begin{array}{c}\text { Early } \\
\text { Discon } \\
\text { Visit }\end{array}$} \\
\hline Intensive PK Arm* & & & & & & & & & & & & & & & & & & & & & & & & \\
\hline Deferred Treatment Arm & & & & & & & & & & & & & & & & & & & & & & & & \\
\hline Visit No. & 1 & 2 & 3 & 4 & 5 & 6 & 7 & 8 & 9 & 10 & 11 & 12 & 13 & 14 & 15 & 16 & 17 & 18 & 19 & 20 & 21 & 22 & 23 & 24 \\
\hline Visit Window & -60 days & $\mathrm{NA}$ & $\begin{array}{c}-6 /+7 \\
\text { days }\end{array}$ & \multicolumn{2}{|c|}{ \pm 1 week } & $\begin{array}{c}-1 /+2 \\
\text { week }\end{array}$ & \multicolumn{3}{|c|}{ \pm 2 week } & \begin{tabular}{|l|}
$-2 /+4$ \\
week
\end{tabular} & $\begin{array}{c} \pm 2 \\
\text { week }\end{array}$ & \multicolumn{3}{|c|}{ \pm 1 week } & \begin{tabular}{|c|}
$-1 /+2$ \\
week
\end{tabular} & \multicolumn{3}{|c|}{ \pm 2 week } & \begin{tabular}{|c|}
$-2 /+4$ \\
week
\end{tabular} & $\begin{array}{c} \pm 2 \\
\text { weeks }\end{array}$ & $\pm 4 \mathrm{w}$ & veeks & NA & \\
\hline DRUG ADMINISTRATION & & & & & & & & & & & & & & & & & & & & & & & & \\
\hline $\begin{array}{l}\text { MK-5172 or placebo } \\
\text { (blinded) }^{8}\end{array}$ & & $\mathrm{x}$ & $\mathrm{x}$ & $\mathrm{x}$ & $\mathrm{x}$ & $\mathrm{x}$ & $\mathrm{x}$ & $\mathrm{x}$ & $\mathrm{x}$ & $x^{11,12}$ & & & & & & & & & & & & & & \\
\hline MK-8742 or placebo(blinded) ${ }^{8}$ & & $\mathrm{x}$ & $\mathrm{x}$ & $\mathrm{x}$ & $\mathrm{x}$ & $\mathrm{x}$ & $\mathrm{x}$ & $\mathrm{x}$ & $\mathrm{x}$ & $\mathrm{x}^{11,12}$ & & & & & & & & & & & & & & \\
\hline MK-5172 (open label) ${ }^{8}$ & & & & & & & & & & $\mathrm{x}^{11,12}$ & $\mathrm{x}$ & $\mathrm{x}$ & $\mathrm{x}$ & $\mathrm{x}$ & $\mathrm{x}$ & $\mathrm{x}$ & $\mathrm{x}$ & $\mathrm{x}$ & $\mathrm{X}^{11,12}$ & & & & & \\
\hline MK-8742 (open label) ${ }^{8}$ & & & & & & & & & & $\mathrm{x}^{11,12}$ & $\mathrm{x}$ & $\mathrm{x}$ & $\mathrm{x}$ & $\mathrm{x}$ & $\mathrm{x}$ & $\mathrm{x}$ & $\mathrm{x}$ & $\mathrm{x}$ & $\mathrm{x}^{11,12}$ & & & & & \\
\hline
\end{tabular}




\section{NOTE: Subjects in the Immediate treatment arm will have Visits 1-10, be unblinded at Week 16, and will then move to Visits 20-22. Subjects in the Deferred treatment arm will have Visits $1-$} 10, be unblinded at Week 16, and will then have visits 11-22.

"Subjects in the Intensive PK arm will have Visits 1-10 and will then move to Visits 20-22.

1 A comprehensive PE will be done at screening and baseline (Day1). For all other visits a focused PE will be conducted when clinically indicated.

2 Review of Adverse Events should include collecting serious adverse events throughout the study and collecting all adverse events Day 1 (post-dose) through 14 days following the last dose of study drug. Adverse events occurring prior to study drug administration or after study drug discontinuation, as a result of a protocol-specified procedure or intervention, should also be reported.

3 Urinalysis will be obtained on all NDD subjects and when feasible on HD subjects.

4 Female subject who is of childbearing potential. Serum pregnancy tests at screening visit. Routinely use urine pregnancy tests beginning predose on Day 1 , however, a serum pregnancy test can be used for patients on hemodialysis who do not produce enough urine for testing.. The urine pregnancy test results must be provided to the investigator and/or site personnel. Subjects should be instructed to contact the investigator and/or site personnel immediately if the result of the self-pregnancy test is positive.

5 Blood samples will be collected for HCV viral resistance testing at baseline, viral failure confirmation visit, and FU visits. At the same time points, samples will be collected for proteomics, and metabolomics and other exploratory analysis.

6 Blood sample will be collected for IL28B genotyping and genetic analysis for ADME and HLA genes. The sample should be sent as a single whole blood sample and the testing facility will extract DNA and split into 2 aliquots for each analysis.

7 Informed Consent for future biomedical research samples must be obtained before the DNA samples are collected. DNA for analysis should be obtained predose, on Day 1 (or with the next scheduled blood draw), as the last sample drawn, on randomized subjects only, or at a later date as soon as the informed consent is obtained. Any leftover plasma from HCV RNA or leftover plasma for HCV viral resistance and biomarker will be stored for future research if the subject consents to participate in the FBR sub-study.

$8 \quad$ MK-5172 and MK-8742 will be provided on a monthly basis. The site will call the Interactive Voice Response System (IVRS) to obtain component ID assignment.

9 Procedures on Day 1 should be performed prior to the first evening dose unless specified otherwise.

10 If a subject is confirmed viral failure during therapy (i.e. break through), then the sample collection for HCV RNA and Viral Resistance/Biomarker is not needed for the early discontinuation visit.

11 There will be no dispensing of study medication on this day. However, the subject will take their last dose(s) of week 12 on that day.

12 For the Week 12 (Week 28 for deferred treatment arm), all subjects will hold their last evening dose of study medications. Subjects will have predose sample taken the next morning at their study visit.

13 All laboratory sampling should be performed prior to dialysis on days with a scheduled dialysis session.

14 Unblinding will occur at Week 16 of the study after all data through Week 12 and safety follow-up after Week 12 have been completed, data cleaned and queries resolved. If the subject is determined to have received active therapy during the first 12 weeks, the visit at Week16 will be the FU 4 visit and those study procedures should be followed. If the subject was determined to have received placebo during the first 12 weeks, the visit at Week 16 will be counted as Visit 11 (Day 1 of active dosing) and those study visits followed. Unblinding is not applicable to the Intensive PK Arm.

15 Serum cryocrit, C4 complement component, and Rheumatoid factor should be collected for all subjects with cryoglobulinemia reported in their Medical History. Because of stability and shipping issues, these samples should only be collected on a Monday or Tuesday.

16 Confirmation of birth control is only needed for 14 days past last dose of study drugs. 


\subsection{TRIAL PROCEDURES}

\subsection{Trial Procedures}

The Trial Flow Chart - Section 6.0 summarizes the trial procedures to be performed at each visit. Individual trial procedures are described in detail below. It may be necessary to perform these procedures at unscheduled time points if deemed clinically necessary by the investigator.

Furthermore, additional evaluations/testing may be deemed necessary by the investigator and or the Sponsor for reasons related to subject safety. In some cases, such evaluation/testing may be potentially sensitive in nature (e.g., HIV, Hepatitis C, etc.), and thus local regulations may require that additional informed consent be obtained from the subject. In these cases, such evaluations/testing will be performed in accordance with those regulations.

\subsubsection{Administrative Procedures}

\subsubsection{Informed Consent}

The investigator or qualified designee must obtain documented consent from each potential subject or each subject's legally acceptable representative prior to participating in a clinical trial or Future Biomedical Research.

\subsection{General Informed Consent}

Consent must be documented by the subject's dated signature or by the subject's legally acceptable representative's dated signature on a consent form along with the dated signature of the person conducting the consent discussion.

A copy of the signed and dated consent form should be given to the subject before participation in the trial.

The initial informed consent form, any subsequent revised written informed consent form and any written information provided to the subject must receive the IRB/ERC's approval/favorable opinion in advance of use. The subject or his/her legally acceptable representative should be informed in a timely manner if new information becomes available that may be relevant to the subject's willingness to continue participation in the trial. The communication of this information will be provided and documented via a revised consent form or addendum to the original consent form that captures the subject's dated signature or by the subject's legally acceptable representative’s dated signature.

Specifics about a trial and the trial population will be added to the consent form template at the protocol level.

The informed consent will adhere to IRB/ERC requirements, applicable laws and regulations and Sponsor requirements. 


\subsection{Consent and Collection of Specimens for Future Biomedical Research}

The investigator or qualified designee will explain the Future Biomedical Research consent to the subject, answer all of his/her questions, and obtain written informed consent before performing any procedure related to the Future Biomedical Research sub-trial. A copy of the informed consent will be given to the subject.

\subsubsection{Inclusion/Exclusion Criteria}

All inclusion and exclusion criteria will be reviewed by the investigator or qualified designee to ensure that the subject qualifies for the trial.

\subsubsection{Subject Identification Card}

All subjects will be given a Subject Identification Card identifying them as participants in a research trial. The card will contain trial site contact information (including direct telephone numbers) to be utilized in the event of an emergency. The investigator or qualified designee will provide the subject with a Subject Identification Card immediately after the subject provides written informed consent.

\subsubsection{Medical History}

A medical history will be obtained by the investigator or qualified designee.

\subsubsection{Prior and Concomitant Medications Review}

\subsection{Prior Medications}

The investigator or qualified designee will review prior medication use, including any protocol-specified washout requirement, and record prior medication taken by the subject within 30 days before starting the trial.

\subsection{Concomitant Medications}

The investigator or qualified designee will record medication, if any, taken by the subject during the trial.

\subsubsection{Assignment of Screening Number}

All consented subjects will be given a unique screening number that will be used to identify the subject for all procedures that occur prior to randomization or allocation. Each subject will be assigned only one screening number. Screening numbers must not be re-used for different subjects.

Any subject who is screened multiple times will retain the original screening number assigned at the initial screening visit. 


\subsubsection{Assignment of Randomization Number}

All eligible subjects will be randomly allocated and will receive a randomization number. The randomization number identifies the subject for all procedures occurring after randomization. Once a randomization number is assigned to a subject, it can never be reassigned to another subject.

A single subject cannot be assigned more than 1 randomization number.

\subsubsection{Trial Compliance (Medication/Diet/Activity/Other)}

The investigator/study coordinator will give the subject a Study Medication Diary to be completed during the study period. The investigator/study coordinator will be responsible for entering the subject's identification (allocation number), visit number, and the dates before giving the diary card to the subject. The subject will be instructed to record dates/times and the number of tablets or capsules of study drug doses on the diary card for the entire time period. Only the subject should enter information on the diary card. The subject is to return the completed diary card at each scheduled visit. At visits when used/unused study medications are returned, site personnel must verify the accuracy of the dosing diary by comparing entries with amounts of returned study medication. If a discrepancy is noted, investigator/study coordinator must discuss the discrepancy with the subject, and the explanation must be documented. Only the subject shall make any changes to the entries on the diary card. The subject will initial the diary card to confirm that the information is accurate. The investigator/study coordinator will be responsible for transferring the appropriate information from the diary card onto the appropriate case report form.

Interruptions from the protocol specified treatment plan require consultation between the investigator and the Sponsor and written documentation of the collaborative decision on subject management.

\subsubsection{Clinical Procedures/Assessments}

\subsubsection{Physical Examination}

All physical examinations must be performed by the principal investigator or subinvestigator (physician, physician assistant or nurse practitioner).

A complete physical examination, performed at the Screening visit and Day 1 includes the following assessments: general appearance, head, eyes, ears/nose/ throat, neck, lymph nodes, skin, lungs, heart, abdomen, musculoskeletal, and neurologic evaluations. Breast, rectal, and genitourinary/pelvic exams should be performed when clinically indicated. For all other visits, a focused exam will be performed when clinically indicated. Any significant changes between the screening visit and Day 1 should be noted in the Medical History eCRF. Any significant changes after receiving study therapy at Day 1 must be reported as adverse events and entered on the adverse event eCRF. If the subject is discontinued for any reason during the treatment phase, every attempt should be made to perform a final physical examination. 
Protocol/Amendment No.: 052-04

Patients with a diagnosis of cryoglobulinemia at study entry should be specifically evaluated for signs and symptoms of the disease.

\subsubsection{Weight and Height Assessment}

The subject's weight should be assessed as mentioned in the flow chart. Clinically significant changes from Day 1 should also be captured as AEs in the CRF. Weight and height will also be utilized to assess the patients GFR in order to monitor the renal disease status.

\subsubsection{12-Lead ECG}

Special care must be taken for proper lead placement. Subjects should be shaved as necessary for proper lead placement. Subjects should be resting in a semi-recumbent position for at least 10 minutes prior to having ECG readings obtained. However, clinically significant findings from the screening ECG must be captured in the medical history eCRF. For ECGs performed during treatment or during the follow-up period, any clinically significant changes compared with the screening ECG must be captured as AEs.

\subsubsection{Vital Signs}

Vital signs will include heart rate (sitting), blood pressure (sitting), and oral temperature. Subjects should be resting in a semi-recumbent position for at least 10 minutes prior to having vital sign measurements obtained.

Note: Oral temperatures should be taken, but if oral is not possible, tympanic, rectal, and axillary temps may be taken,

After the screening visit, the site should indicate whether or not the result is clinically significant and if any subsequent changes constitute an adverse event.

\subsubsection{Birth Control Confirmation}

Confirmation must be obtained by site personnel that subjects and their partner(s) are using acceptable methods of contraception. This assessment must be documented in the subject's study chart at each specified visit.

\subsubsection{Adverse Events}

The principal investigator or sub-investigator (physician, physician assistant or nurse practitioner) must determine the severity and relationship to study medication(s) of all adverse events. A physician investigator must review, initial and date the severity of all adverse events and their relationship to study medications when initial assessment of an adverse event is made by a physician assistant or nurse practitioner. Designated medical practitioners must be licensed and the responsibilities transferred to them must be documented in the site file. For details please refer to Section 7.2 


\subsubsection{Noninvasive Methods of Cirrhosis Evaluation}

FibroScan - This method for assessing liver cirrhosis has gained increasing acceptance. In the US, this methodology is FDA approved and in other countries it is often the preferred method of assessment. Fibroscan results are influenced by a number of confounders including ALT, ascites, and underlying disease. Hepatitis $\mathrm{C}$ is one of the best studied and is the disease state with the most reproducible/reliable results. Fibroscan has been evaluated in many liver diseases for the staging of liver fibrosis, and has been demonstrated to be very effective or differentiating cirrhosis (F4) from no cirrhosis $(<\mathrm{F} 4)$, but it is less capable of differentiating gradations of fibrosis. In a large study by Castera, et al [42], a population of patients with chronic hepatitis $\mathrm{C}$, a cut-off of $12.5 \mathrm{kPa}$ was selected for cirrhotics. At this cut-off, the sensitivity and specificity of the test for cirrhosis were $87 \%$ and $91 \%$, respectively and the negative predictive value was $95 \%$. Since this analysis was assessed specifically in patients with chronic hepatitis $\mathrm{C}$, the cut-off value $\leq 12.5 \mathrm{kPa}$ used by Castera was selected to exclude cirrhotics in the current study.

FibroTest + APRI - Various methodologies have been developed in order to improve the sensitivity and specificity of blood tests used to diagnose cirrhosis in patients with chronic hepatitis $\mathrm{C}$ infections. One such algorithm, the Sequential Algorithm for Fibrosis Evaluation (SAFE), which uses a combination of Fibrotest and the aspartate aminotransferase-to platelet ratio index (APRI) is very accurate for diagnosing cirrhosis [43]. For cirrhosis, the SAFE for F4 algorithm provides a diagnostic accuracy of $89.5 \%$ with a negative predictive value of $94.6 \%$. Using this algorithm, it is estimated that only $6.2 \%$ of the patients would need a liver biopsy to confirm the diagnosis of cirrhosis. The cut-off values for excluding cirrhotics using the two tests, without the use of liver biopsy, are $\leq 1$ and $\leq 0.48$ for FibroTest and APRI when the SAFE for F4 is used. This study uses this method with one variation and that is the more stringent requirement that both the APRI and FibroTest need to be consistent with no cirrhosis, i.e. APRI is $\leq 1$ AND Fibrotest $\leq 0.48$. Accordingly, the Sponsor is confident these cut-off values that will differentiate cirrhotic from non-cirrhotic patients with reasonable accuracy in this study.

\subsubsection{Patient-Reported Outcomes}

\section{SF36v2® Health Survey}

Health-related quality of life will be assessed using the SF-36v2® Health Survey, Acute (1week recall) Form, a generic health survey, which includes 36 questions to measure functional health and well-being from the patient's perspective. The SF-36v2 ${ }^{\circledR}$ measures each of the following eight health domains: Physical Functioning, Role Limitations-Physical, Bodily Pain, General Health, Vitality, Social Functioning, Role Limitations-Emotional, and Mental Health. The eight health domain scores contribute to the computation of the Physical Component Summary (PCS) and Mental Component Summary (MCS) scores.

Subjects will be administered the SF-36v2 ${ }^{\circledR}$ on an electronic device and are to complete the SF-36v2 ${ }^{\circledR}$ on their own at the beginning of the appropriate study visit [Day 1, Week 12, Week 28 (deferred treatment arm only), Follow-Up Week 12, and Early Discontinuation]. 
Every attempt should be made to complete the questionnaires prior to receiving study treatment, discussing any medical conditions, or receiving any medical results (see study flow chart). It should take subjects approximately 5-10 minutes to complete the SF-36v2 ${ }^{\circledR}$.

\subsubsection{Laboratory Procedures/Assessments}

Details regarding specific laboratory procedures/assessments to be performed in this trial are provided below. The total amount of blood/tissue to be drawn/collected over the course of the trial (from pre-trial to post-trial visits), including approximate blood/tissue volumes drawn/collected by visit and by sample type per subject can be found in Section 12.4.

NOTE: All laboratory sampling should be performed prior to dialysis on days with a scheduled dialysis session.

\subsubsection{Laboratory Safety Evaluations (Hematology, Chemistry and Urinalysis)}

Laboratory tests for hematology, chemistry and urinalysis are specified in Table 6.

NOTE: For subjects on hemodialysis, samples should be drawn prior to any scheduled HD. 
Protocol/Amendment No.: 052-04

Table 6 Laboratory Tests

\begin{tabular}{|c|c|c|c|}
\hline Hematology & Chemistry & Urinalysis & Other \\
\hline Hematocrit & Albumin & Specific Gravity & Hemoglobin A1C (HbA1c) \\
\hline Hemoglobin & Alkaline phosphatase & $\mathrm{pH}$ & Hepatitis C Virus Genotype \\
\hline Platelet count & Alanine aminotransferase (ALT) & Glucose & Plasma HCV RNA \\
\hline $\begin{array}{l}\text { WBC (total and } \\
\text { differential) }\end{array}$ & $\begin{array}{l}\text { Aspartate aminotransferase } \\
\text { (AST) }\end{array}$ & Protein & Prothrombin time (PT) \\
\hline \multirow[t]{14}{*}{$\begin{array}{l}\text { Erythrocytes } \\
\text { (RBC count) }\end{array}$} & Creatinine & Ketones & $\begin{array}{l}\text { International normalized } \\
\text { Ratio (INR) }\end{array}$ \\
\hline & $\begin{array}{l}\text { Creatinine Clearance (for CKD } \\
\text { patients throughout) }\end{array}$ & Occult Blood & $\begin{array}{l}\text { Human Chorionic } \\
\text { gonadotropin } \\
\text { (Urine pregnancy test kits to } \\
\text { sites) }\end{array}$ \\
\hline & Creatine Kinase & Bilirubin & $\begin{array}{l}\text { HIV-1 serology (screening } \\
\text { only) }\end{array}$ \\
\hline & Gamma-glutamyltransferase & Nitrite & HBsAg (screening only) \\
\hline & Glucose (serum glucose) & Leukocytes & $\begin{array}{l}\text { APRI calculation (screening } \\
\text { only) }\end{array}$ \\
\hline & Potassium & Erythrocytes & $\begin{array}{l}\text { Fibrosure }{ }^{\circledR} \text { (Fibrotest) as } \\
\text { requested by site for entry } \\
\text { critiera (may be performed } \\
\text { locally) }\end{array}$ \\
\hline & Sodium & $\begin{array}{l}\text { Microscopic exam, if } \\
\text { abnormal results are } \\
\text { noted }\end{array}$ & eGFR calculation \\
\hline & Total Bilirubin & & \\
\hline & Direct Bilirubin & & $\begin{array}{l}\text { For subjects with } \\
\text { cryoglobulinemia at study } \\
\text { entry: } \\
\text { - Serum cryocrit } \\
\quad \text { (cryoglobulin level) }\end{array}$ \\
\hline & Indirect Bilirubin & & - Rheumatoid factor \\
\hline & Total protein & & $\begin{array}{l}\text { - C4 Complement } \\
\text { component }\end{array}$ \\
\hline & Blood Urea Nitrogen & & \\
\hline & Amylase & & \\
\hline & Lipase & & \\
\hline
\end{tabular}

\subsubsection{Pharmacokinetic/Pharmacodynamic Evaluations}

The decision as to which plasma samples collected will be assayed for evaluation of pharmacokinetics/pharmacodynamics will be collaboratively determined by the Departments of Quantitative Pharmacology and Pharmacometrics (QPP) and the appropriate department within Late-Stage Development. If indicated, these samples may also be assayed and/or pooled for assay in an exploratory manner for metabolites and/or additional pharmacodynamic markers. 
Protocol/Amendment No.: 052-04

\subsection{Blood Collection for Plasma MK-5172 and MK-8742}

Sample collection, storage and shipment instructions for plasma samples will be provided in the operations/laboratory manual.

All subjects allocated and enrolled in the study (all 3 treatment arms) will be part of the population PK group (i.e. sparse PK sampling scheme). See Table 7 for sampling scheme. For the Week 12 sampling, all subjects will withhold their last evening dose of study medications. Subjects will have a predose sample taken the next morning at their study visit, subjects will then take their study medications, and have a 2 hour post dose sample taken. For the Week 28 sampling, subjects in the deferred treatment arm will hold their last evening dose of study medications. Subjects will have a predose sample taken the next morning at their study visit, subjects will then take their study medications, and have a 2 hour post dose sample taken.

Five (5) HD subjects and five (5) subjects not on HD will be allocated to an intensive PK subgroup receiving open-label MK-5172 in combination with MK-8742. See Table 8 for sampling scheme. At Week 4, these 10 subjects will take their study medications in the PM and be domiciled overnight for the sample collections. The 24-hour PK collection in HD and non-HD subjects (Week 4) will be used to characterize the representative steady-state exposures in both populations.

An additional ten (10) HD subjects and ten (10) non-dialysis subjects will be identified from the immediate and deferred treatment arms to participate in an evening pre-dose PK cohort, but only the samples from the subjects on active treatment (immediate treatment arm) will be analyzed. See Table 9 for sampling scheme. A visiting nurse will collect a pre-dose sample at the protocol specified Week 4 PK timepoint prior to their PM dose. The predose PK sample collected in the evening pre-dose PK cohort will be used to correlate the PK exposures between PK samples collected in the AM (e.g., Week 12, 28) and the PM (e.g., Week 4).

All PK samples (from population PK, intensive PK, and evening pre-dose PK) will be used to evaluate not only PK exposures in the non-dialysis CKD and HD populations, but also to assess the PK/PD and PK/AE relationships of MK-5172 and MK-8742, as appropriate. 
Product: MK-5172

Protocol/Amendment No.: 052-04

Table 7 Pharmacokinetic Sampling Timepoints- Population PK (All Subjects)

\begin{tabular}{|c|c|c|c|c|c|}
\hline $\begin{array}{c}\text { Visit } \\
\text { Numbe } \\
\text { r }\end{array}$ & $\begin{array}{c}\text { Study } \\
\text { Population }\end{array}$ & Study Day/Week & $\begin{array}{c}\text { Time Relative to Dose of } \\
\text { MK-5172, MK-8742 }\end{array}$ & $\begin{array}{c}\text { MK-5172 } \\
\text { PK Sample }^{1}\end{array}$ & $\begin{array}{c}\text { MK-8742 } \\
\text { PK Sample }^{1}\end{array}$ \\
\hline 2 & $\begin{array}{c}\text { All } 3 \\
\text { treatment } \\
\text { arms }\end{array}$ & Day 1 & Predose & $\mathrm{x}$ & $\mathrm{x}$ \\
\hline \multirow{2}{*}{10} & \multirow{2}{*}{$\begin{array}{c}\text { All } 3 \\
\text { treatment } \\
\text { arms }\end{array}$} & \multirow{2}{*}{ Week 12} & Predose & $\mathrm{X}$ & $\mathrm{X}$ \\
\hline & & & 2 hrs Postdose & $\mathrm{x}$ & $\mathrm{x}$ \\
\hline \multirow{4}{*}{19} & Deferred & \multirow[b]{2}{*}{ Week 28} & Predose & $\mathrm{x}$ & $\mathrm{X}$ \\
\hline & $\begin{array}{l}\text { Treatment } \\
\text { Arm Only }\end{array}$ & & $\sim 2$ hrs Postdose & $\mathrm{x}$ & $\mathrm{x}$ \\
\hline & $\begin{array}{c}\text { Not } \\
\text { applicable }\end{array}$ & $\begin{array}{c}\text { Unsched/Viral } \\
\text { Failure Conf } \\
\text { Visit } \\
\end{array}$ & $\mathrm{NA}^{3}$ & $\mathrm{x}$ & $\mathrm{x}$ \\
\hline & $\begin{array}{c}\text { Not } \\
\text { applicable }\end{array}$ & $\begin{array}{l}\text { Early Discon } \\
\text { Visit } \\
\end{array}$ & $\mathrm{NA}^{3}$ & $\mathrm{X}$ & $\mathrm{X}$ \\
\hline \multicolumn{6}{|c|}{$\begin{array}{l}1 \text { mL of blood will be collected at each specified time point for plasma PK assessments of MK-5172 } \\
\text { and MK-8742. } \\
2 \text { Time Relative to last Dose of MK-5172, MK-8742 must be recorded in INFORM } \\
3 \text { The date and time of the last MK-5172, MK-8742 dose prior to all PK sample collection must be } \\
\text { recorded in INFORM } \\
\text { Note: At the time of PK sample collection, subjects will be asked to provide information regarding the } \\
\text { time/date of the last MK-5172, MK-8742 dose prior to the PK sample collection. (This can also be } \\
\text { obtained by referencing the subject's study medication diary). }\end{array}$} \\
\hline
\end{tabular}


Protocol/Amendment No.: 052-04

Table 8 Pharmacokinetic Sampling Timepoints- Intensive PK Arm

\begin{tabular}{|c|c|c|c|c|}
\hline $\begin{array}{c}\text { Visit } \\
\text { Number }\end{array}$ & $\begin{array}{c}\text { Study } \\
\text { Day/Week }\end{array}$ & $\begin{array}{c}\text { Time Relative to Dose of MK-5172, } \\
\text { MK- } 8742^{2}\end{array}$ & $\begin{array}{l}\text { MK-5172 PK } \\
\text { Sample }^{1}\end{array}$ & $\begin{array}{c}\text { MK-8742 } \\
\text { PK Sample }\end{array}$ \\
\hline 2 & Day $1^{4}$ & Predose & $\mathrm{x}$ & $\mathrm{x}$ \\
\hline \multirow{11}{*}{6} & \multirow{11}{*}{ Week 4} & Predose & $\mathrm{x}$ & $\mathrm{X}$ \\
\hline & & 0.5 & $\mathrm{x}$ & $\mathrm{X}$ \\
\hline & & 1 & $\mathrm{x}$ & $\mathrm{x}$ \\
\hline & & 2 & $\mathrm{x}$ & $\mathrm{X}$ \\
\hline & & 3 & $\mathrm{x}$ & $\mathrm{X}$ \\
\hline & & 4 & $\mathrm{x}$ & $\mathrm{x}$ \\
\hline & & 6 & $\mathrm{x}$ & $\mathrm{x}$ \\
\hline & & 8 & $\mathrm{x}$ & $\mathrm{X}$ \\
\hline & & 12 & $\mathrm{x}$ & $\mathrm{X}$ \\
\hline & & 16 & $\mathrm{x}$ & $\mathrm{x}$ \\
\hline & & 24 & $\mathrm{x}$ & $\mathrm{X}$ \\
\hline \multirow[t]{4}{*}{10} & \multirow[t]{2}{*}{ Week $12^{4}$} & Predose & $\mathrm{X}$ & $\mathrm{X}$ \\
\hline & & $\sim 2$ hrs Postdose & $\mathrm{x}$ & $\mathrm{X}$ \\
\hline & $\begin{array}{l}\text { Unsched/Viral } \\
\text { Failure Conf } \\
\text { Visit }\end{array}$ & $\mathrm{NA}^{3}$ & $\mathrm{x}$ & $\mathrm{X}$ \\
\hline & $\begin{array}{c}\text { Early Discon } \\
\text { Visit }\end{array}$ & $\mathrm{NA}^{3}$ & $\mathrm{x}$ & $\mathrm{x}$ \\
\hline \multirow{4}{*}{\multicolumn{5}{|c|}{$\begin{array}{l}1 \text { m } ~ m \text { of blood will be collected at each specified time point for plasma PK assessments of MK-5172 } \\
\text { and MK-8742. } \\
2 \text { Time Relative to last Dose of MK-5172, MK-8742 must be recorded in INFORM } \\
3 \text { The date and time of the last MK-5172, MK-8742 dose prior to each PK sample collected must be } \\
\text { recorded in INFORM } \\
{ }^{4} \text { Since all subjects will have samples taken on Day } 1 \text { and on Week } 12 \text { for population PK, additional } \\
\text { samples for Intensive PK do not need to be collected. }\end{array}$}} \\
\hline & & & & \\
\hline & & & & \\
\hline & & & & \\
\hline \multicolumn{5}{|c|}{$\begin{array}{l}\text { Note: At the time of PK sample collection, subjects will be asked to provide information regarding the } \\
\text { time/date of the last MK-5172, MK- } 8742 \text { dose prior to the PK sample collection. (This can also be } \\
\text { obtained by referencing the subject's study medication diary). }\end{array}$} \\
\hline
\end{tabular}


Protocol/Amendment No.: 052-04

Table 9 Pharmacokinetic Sampling Timepoints- Evening Pre-Dose PK Cohort

\begin{tabular}{|c|c|c|c|c|c|}
\hline $\begin{array}{l}\text { Visit } \\
\text { Numbe } \\
\text { r }\end{array}$ & $\begin{array}{c}\text { Study } \\
\text { Population }\end{array}$ & Study Day/Week & $\begin{array}{l}\text { Time Relative to Dose of } \\
\text { MK-5172, MK-8742 }\end{array}$ & $\begin{array}{c}\text { MK-5172 } \\
\text { PK Sample }\end{array}$ & $\begin{array}{l}\text { MK-8742 } \\
\text { PK Sample }^{1}\end{array}$ \\
\hline 6 & $\begin{array}{c}\text { Immediate } \\
\text { and Deferred } \\
\text { Treatment } \\
\text { arm } \\
\end{array}$ & Week 4 & Predose & $\mathrm{x}$ & $\mathrm{X}$ \\
\hline \multirow[t]{2}{*}{10} & \multirow{2}{*}{$\begin{array}{c}\text { Immediate } \\
\text { and Deferred } \\
\text { Treatment } \\
\text { arm }\end{array}$} & \multirow[t]{2}{*}{ Week $12^{4}$} & Predose & $\mathrm{X}$ & $\mathrm{X}$ \\
\hline & & & $\sim 2$ hrs Postdose & $\mathrm{X}$ & $\mathrm{X}$ \\
\hline & $\begin{array}{c}\text { Not } \\
\text { applicable }\end{array}$ & $\begin{array}{l}\text { Unsched/Viral } \\
\text { Failure Conf } \\
\text { Visit }\end{array}$ & $\mathrm{NA}^{3}$ & $\mathrm{x}$ & $\mathrm{X}$ \\
\hline & $\begin{array}{c}\text { Not } \\
\text { applicable }\end{array}$ & $\begin{array}{l}\text { Early Discon } \\
\text { Visit }\end{array}$ & $\mathrm{NA}^{3}$ & $\mathrm{x}$ & $\mathrm{X}$ \\
\hline \multicolumn{6}{|c|}{${ }^{1} \sim 4 \mathrm{~mL}$ of blood will be collected at each specified time point for plasma PK assessments of MK-5172 } \\
\hline \multirow{2}{*}{\multicolumn{6}{|c|}{$\begin{array}{l}\text { Time Relative to last Dose of MK-5172, MK-8742 must be recorded in INFORM } \\
\text { The date and time of the last MK-5172, MK-8742 dose prior to each PK sample collected must be } \\
\text { recorded in INFORM }\end{array}$}} \\
\hline & & & & & \\
\hline \multicolumn{6}{|c|}{$\begin{array}{l}{ }^{4} \text { Since all subjects will have samples taken on Week } 12 \text { for population PK, additional samples for evening } \\
\text { pre-dose PK do not need to be collected }\end{array}$} \\
\hline \multicolumn{6}{|c|}{$\begin{array}{l}\text { Note: At the time of PK sample collection, subjects will be asked to provide information regarding the } \\
\text { time/date of the last MK-5172, MK- } 8742 \text { dose prior to the PK sample collection. (This can also be } \\
\text { obtained by referencing the subject's study medication diary). }\end{array}$} \\
\hline
\end{tabular}

\subsubsection{Future Biomedical Research}

The following specimens are to be obtained as part of Future Biomedical Research:

- Blood for genomics use

- Leftover plasma from HCV RNA

- Leftover plasma from viral resistance and biomarkers

\subsubsection{HCV Evaluation}

The following specimens are to be obtained as part of Efficacy/Pharmacogenetic Measurements:

- Samples for HCV Genotype evaluation must be obtained as part of the main consent for inclusion in the study.

- Blood must be drawn from each subject as part of the main consent to assess HCV RNA plasma levels at various time points as shown in the flow chart. HCV-RNA in 
plasma will be measured using a COBAS ${ }^{\mathrm{TM}}$ AmpliPrep/COBAS ${ }^{\mathrm{TM}}$ Taqman $^{\mathrm{TM}} \mathrm{HCV}$ Test, v2.0 ${ }^{\circledR}$ assay. Lleftover plasma may be used for future biomedical research only if the subject signed for future biomedical consent.

- Blood must be drawn from each subject as part of the main consent to assess viral resistance mutation and processed as instructed by the central laboratory manual.

- Protein and metabolites may be measured from blood samples to compare biomarkers measured prior to treatment, to biomarkers measured at several time points during treatment that correlate with subject response to treatment (sustained viral response).

- Samples collected for IL28B genotyping and genetic analysis for ADME and HLA genes associated with liver injury are obtained at Day 1 as part of the main consent. The assay performed is specific to the $I L 28 B$ gene region and genes related to HLA and ADME. Any remaining specimen after the genetic analysis has been performed will be destroyed.

Note: Samples may also be used for future assay development and validation

\subsubsection{Other Procedures}

\subsubsection{Withdrawal/Discontinuation}

Subjects who discontinue/withdraw from treatment prior to completion of the treatment regimen should be encouraged to continue to be followed for all remaining study visits.

\subsection{Withdrawal From Future Biomedical Research}

Subjects may withdraw their consent for Future Biomedical Research and have their specimens and all derivatives destroyed. Subjects may withdraw consent at any time by contacting the principal investigator for the main trial. If medical records for the main trial are still available, the investigator will contact the Sponsor using the designated mailbox (clinical.specimen.management@merck.com), and a form will be provided by the Sponsor to obtain appropriate information to complete specimen withdrawal. Subsequently, the subject's specimens will be removed from the biorepository and be destroyed. A letter will be sent from the Sponsor to the investigator confirming the destruction. It is the responsibility of the investigator to inform the subject of completion of destruction. Any analyses in progress at the time of request for destruction or already performed prior to the request being received by the Sponsor will continue to be used as part of the overall research trial data and results. No new analyses would be generated after the request is received.

In the event that the medical records for the main trial are no longer available (e.g., if the investigator is no longer required by regulatory authorities to retain the main trial records) or the specimens have been completely anonymized, there will no longer be a link between the subject's personal information and their specimens. In this situation, the request for specimen destruction cannot be processed. 


\subsubsection{Blinding/Unblinding}

IVRS/IWRS should be used for emergency unblinding in the event that this is required for subject safety. The emergency unblinding call center will provide after-hours emergency unblinding coverage when the investigator is not available.

In the event that unblinding has occurred, the circumstances around the unblinding (e.g., date and reason) must be documented promptly, and the Sponsor Clinical Director notified as soon as possible. Only the principal investigator or delegate and the respective subject's code should be unblinded. Trial site personnel and Sponsor personnel directly associated with the conduct of the trial should not be unblinded.

\subsubsection{Calibration of Critical Equipment}

The investigator or qualified designee has the responsibility to ensure that any critical device or instrument used for a clinical evaluation/test during a clinical trial that provides important information about inclusion/exclusion criteria and/or safety or efficacy parameters shall be suitably calibrated and maintained to ensure that the data obtained is reliable and/or reproducible. Documentation of equipment calibration must be retained with the study documentation as source documentation at the trial site.

Critical Equipment for this trial includes:

None

\subsubsection{Rescreening}

- Subjects who have previously completed the screening visit (Visit 1) and were deemed eligible for randomization into this study, but failed to be randomized within the 60-day window, may be rescreened to re-evaluate study eligibility. To reconfirm the subject's eligibility, all pre-study evaluations should be repeated, after approval from the SPONSOR, except for the following:

- HCV GT Determination

- Liver biopsy

- 12-Lead ECG

If any of the laboratory exclusion criteria are met, the site may have the abnormal value retested one time.

\subsubsection{PK Sampling Time Points}

Subjects must follow the protocol defined specific time points for predose or post dose in respect to study medication administration for PK sample collection. If predose PK sample is required by the protocol, the subject should withhold their dose the day of PK sample. For 
Protocol/Amendment No.: 052-04

detailed time points of PK sample collection please refer to Table 7, Table 8 and Table 9 in Section 7.1.4.2.1.

\subsubsection{Visit Requirements}

Visit requirements are outlined in Section 6.0 - Trial Flow Chart. Specific procedure-related details are provided above in Section 7.1 - Trial Procedures.

\subsubsection{Screening}

Within 60 days prior to administration of the initial dose of study drug, potential subjects will be evaluated to determine that they fulfill the entry requirements as set forth in Section 5.1. Verification should be obtained to confirm that the subject is non-cirrhotic and the subject's fibrosis score must be captured to support secondary data analysis. The primary etiology of the subject's renal disease must be captured. The investigator will discuss with each potential subject the nature of the study, its requirements, and its restrictions.

Subjects will be instructed that they are required to use two acceptable methods of birth control from at least 2 weeks prior to Day 1 and throughout treatment, or longer if dictated by local regulations, after the last dose of study medication.

Subjects will be instructed about the restrictions for concomitant medications, as noted in Section 5.5.

All screening procedures listed for Visit 1 in the Study Flow Chart must be completed and subject eligibility confirmed by the investigator prior to the subject's randomization and drug administration.

All subjects will be given a card, at the time of screening, identifying them as participants in a research study. The card will contain contact information (including direct telephone numbers) to be utilized in the event of an emergency.

\subsubsection{Treatment Period Visit}

\section{Treatment Day 1 (Visit 2)}

\section{Pretreatment Procedures}

Day 1 procedures listed on the Study Flow Chart should be performed prior to dosing unless specified otherwise. For female subjects, a urine pregnancy test will be performed at the site prior to study drug initiation. If the urine pregnancy test result is negative, the subject will be eligible for randomization and the remainder of the pretreatment (Day 1) testing/procedures will be performed. If the urine pregnancy test result is positive, the subject must not be randomized. 
Blood will be collected for assay of safety evaluations, plasma HCV RNA, and PK measurements. These samples will be sent to the appropriate central laboratory(ies) following the procedure(s) set forth in the manual(s).

Additional samples will be collected for genetic evaluation of host parameters related to the response of HCV subjects to MK-5172 and MK-8742 therapies.

\subsubsection{Drug Administration}

Following completion of the Day 1 procedures and confirmation of eligibility, the site pharmacist or study coordinator will contact the IVRS for assignment of the drug to be administered. Sites should not call IVRS for drug administration until the subject has met all criteria for the study and are ready to receive the first dose of study medication on Day 1.

The first dose of trial treatment will be taken by the subject in the evening of Day 1 (Visit 2) for all subjects (Immediate Treatment, Deferred Treatment and Intensive PK arms) and in the evening of the Week 16 visit (Visit 10) only for subjects in the deferred treatment arm. Subsequent dosing will be taken in the evenings by the subject at approximately the same time each day (except for Week 12 and Week 28 doses which must be withheld the night before for the predose PK collection at those visits).

Subjects who discontinue therapy in the trial prior to the last scheduled treatment visit should have an Early Discontinuation visit and then continue into follow-up visits.

At a minimum, collect the following information when a subject discontinues:

1. The reason the subject discontinued.

2. The date of the last dose of study medications from the trial.

3. The date of the last assessment and/or contact. A follow-up contact (telephone or visit) will be arranged as appropriate.

4. (Serious) Adverse events.

5. Final Assessments: Every effort should be made to ensure that all procedures and evaluations scheduled for the Early Discon Visit are performed.

6. Retrieve all study medications from the subject.

\subsubsection{Follow-Up Visits}

At the completion of study therapy (see Section 5.6) subjects will return to the study site for follow-up visits at 4, 12, and 24 weeks, after the last dose of study drug. If a subject completes 12 weeks of therapy (immediate treatment arm and intensive PK arm), the 4, 12, and 24 -week follow-up visits will occur approximately 16, 24, and 36 weeks after Day 1, respectively. Similarly, if a subject completes 24 weeks of therapy (deferred treatment arm), 
the 4, 12, and 24 -week follow-up visits will occur approximately 28, 36, and 48 weeks after Day 1 , respectively.

Subjects who discontinue because they have met criteria for virologic failure while on study therapy should complete an Early Discontinuation Visit as outlined in the Study Flow Chart (Section 6), and return to the study site for follow-up visits at 4, 12, and 24 weeks following the confirmation of virologic failure. Subjects who meet the virologic failure criterion of relapse (having HCV RNA $\geq$ LLoQ following end of all study therapy, after becoming undetectable (TND) at end of treatment) will return to the study site for follow-up visits at 4 , 12, and 24 weeks as outlined in the Study Flow Chart (Section 6).

Subjects who discontinue for reasons other than virologic failure should complete an Early Discontinuation Visit as outlined in the Study Flow Chart and return to the study site for follow-up visits at 4, 12, and 24 weeks following the discontinuation of treatment.

\section{Follow-up after Trial Completion}

All subjects who have taken at least one dose of MK-5172 or MK-8742 will be asked to consent to a follow-up protocol (MK-5172 Protocol 017, a 3 year follow-up program to study efficacy and/or resistance associated variants to any compound used in a MK-5172 treatment regimen). Subjects included in this follow-up protocol may include subjects who have initiated other HCV treatments i.e. rescue or other clinical trials, subjects who failed therapy in this trial who do not want to initiate a new HCV treatment and subjects who achieved viral remission during this trial. Subjects who undergo kidney transplant and either complete or discontinue this study will also be followed in PN017. The purpose of this follow-up protocol is to follow resistance associated variants (RAVs) over time and in the case of treatment responders, to follow durability of response.

\subsubsection{Evaluations of Laboratory Safety Signals}

Laboratory safety measurements will be evaluated weekly throughout the study to assess potential liver safety signals.

If a subject has one or more of the laboratory ECI criteria (Refer section 7.2.3.2) at the last dosing visit (Week 12 for immediate treatment arm or intensive PK arm or Week 24 for deferred treatment arm), then the subject should return to the site weekly for additional monitoring until the values normalize.

\subsubsection{Trial Unblinding}

For emergency unblinding please refer to Section 7.1.4.4.

Unblinding will occur at Week 16 of the study after all data through Week 12 and safety follow-up after Week 12 have been completed, data cleaned and queries resolved. If the subject is determined to have received active therapy during the first 12 weeks, the visit at Week 16 will be the FU 4 visit and those study procedures should be followed. If the subject 
was determined to have received placebo during the first 12 weeks, the visit at Week 16 will be counted as Visit 11 (Day 1 of active dosing) and those study visits followed.

\subsection{Assessing and Recording Adverse Events}

An adverse event is defined as any untoward medical occurrence in a patient or clinical investigation subject administered a pharmaceutical product and which does not necessarily have to have a causal relationship with this treatment. An adverse event can therefore be any unfavourable and unintended sign (including an abnormal laboratory finding, for example), symptom, or disease temporally associated with the use of a medicinal product or protocolspecified procedure, whether or not considered related to the medicinal product or proto colspecified procedure. Any worsening (i.e., any clinically significant adverse change in frequency and/or intensity) of a preexisting condition that is temporally associated with the use of the Sponsor's product, is also an adverse event.

Changes resulting from normal growth and development that do not vary significantly in frequency or severity from expected levels are not to be considered adverse events. Examples of this may include, but are not limited to, teething, typical crying in infants and children and onset of menses or menopause occurring at a physiologically appropriate time.

Sponsor's product includes any pharmaceutical product, biological product, device, diagnostic agent or protocol-specified procedure, whether investigational (including placebo or active comparator medication) or marketed, manufactured by, licensed by, provided by or distributed by the Sponsor for human use.

Adverse events may occur during the course of the use of the Sponsor's product in clinical trials or within the follow-up period specified by the protocol, or prescribed in clinical practice, from overdose (whether accidental or intentional), from abuse and from withdrawal.

Adverse events may also occur in screened subjects during any pre-allocation baseline period as a result of a protocol-specified intervention, including washout or discontinuation of usual therapy, diet, placebo treatment or a procedure.

All adverse events will be recorded from the time the consent form is signed through 14 days following cessation of treatment and at each examination on the Adverse Event case report forms/worksheets. The reporting timeframe for adverse events meeting any serious criteria is described in section 7.2.3.1.

\subsubsection{Definition of an Overdose for This Protocol and Reporting of Overdose to the Sponsor}

In this trial, an overdose is any dose higher than: Any intake in excess of the prescribed dose of MK-5172 or MK-8742 per calendar day.

If an adverse event(s) is associated with ("results from") the overdose of Sponsor's product or vaccine, the adverse event(s) is reported as a serious adverse event, even if no other seriousness criteria are met.

MK-5172-052-04 Final Protocol

04-Jun-2014 
If a dose of Sponsor's product or vaccine meeting the protocol definition of overdose is taken without any associated clinical symptoms or abnormal laboratory results, the overdose is reported as a non-serious Event of Clinical Interest (ECI), using the terminology "accidental or intentional overdose without adverse effect."

All reports of overdose with and without an adverse event must be reported within 24 hours to the Sponsor either by electronic media or paper. Sponsor Contact information can be found in the Investigator Trial File Binder (or equivalent).

\subsubsection{Reporting of Pregnancy and Lactation to the Sponsor}

Although pregnancy and lactation are not considered adverse events, it is the responsibility of investigators or their designees to report any pregnancy or lactation in a subject (spontaneously reported to them), including the pregnancy of a male subject's female partner that occurs during the trial or within 14 days of completing the trial. All subjects and female partners of male subjects who become pregnant must be followed to the completion/termination of the pregnancy. Pregnancy outcomes of spontaneous abortion, missed abortion, benign hydatidiform mole, blighted ovum, fetal death, intrauterine death, miscarriage and stillbirth must be reported as serious events (Important Medical Events). If the pregnancy continues to term, the outcome (health of infant) must also be reported.

Such events must be reported within 24 hours to the Sponsor either by electronic media or paper. Sponsor Contact information can be found in the Investigator Trial File Binder (or equivalent).

\subsubsection{Immediate Reporting of Adverse Events to the Sponsor}

\subsubsection{Serious Adverse EventsAdverse Events and Incidents}

A serious adverse event is any adverse event occurring at any dose or during any use of Sponsor's product that:

- Results in death;

- Is life threatening;

- Results in persistent or significant disability/incapacity;

- Results in or prolongs an existing inpatient hospitalization;

- Is a congenital anomaly/birth defect;

- Is a cancer;

- Is associated with an overdose;

- Is an other important medical event

Refer to Table 10 for additional details regarding each of the above criteria.

Any serious adverse event, or follow up to a serious adverse event, including death due to any cause that occurs to any subject from the time the consent is signed through 14 days following cessation of treatment or within the established off therapy follow-up period for safety described in the protocol, whether or not related to the Sponsor's product, must be 
reported within 24 hours to the Sponsor either by electronic media or paper. Sponsor Contact information can be found in the Investigator Trial File Binder (or equivalent).

Additionally, any serious adverse event, considered by an investigator who is a qualified physician to be related to the Sponsor's product that is brought to the attention of the investigator at any time outside of the time period specified in the previous paragraph also must be reported immediately to the Sponsor.

All subjects with serious adverse events must be followed up for outcome.

\subsubsection{Events of Clinical Interest}

Selected non-serious and serious adverse events are also known as Events of Clinical Interest (ECI) and must be recorded as such on the Adverse Event case report forms/worksheets and reported within 24 hours to the Sponsor either by electronic media or paper. Sponsor Contact information can be found in the Investigator Trial File Binder (or equivalent).

Events of clinical interest for this trial include:

1. an overdose of Sponsor's product, as defined in Section 7.2.1 - Definition of an Overdose for This Protocol and Reporting of Overdose to the Sponsor, that is not associated with clinical symptoms or abnormal laboratory results.

2. first instance of ALT or AST $>500 \mathrm{IU} / \mathrm{L}$ from the initiation of study therapy through 14 days following treatment and not associated with virologic failure*

3. first instance of ALT or AST>3x baseline AND >100 IU/L from the initiation of study therapy through 14 days following treatment and not associated with virologic failure*

4. first instance of alkaline phosphatase>3x ULN from the initiation of study therapy through 14 days following treatment and not associated with virologic failure*

*Note: The purpose of the criteria is to specify a threshold of abnormal hepatic tests that may require an additional evaluation for an underlying etiology. The trial site guidance for assessment and follow up of these criteria can be found in the Investigator Trial File Binder (or equivalent).

\subsubsection{Protocol-Specific Exceptions to Serious Adverse Event Reporting}

\subsubsection{Evaluating Adverse Events}

An investigator who is a qualified physician will evaluate all adverse events with respect to the elements outlined in Table 10. The investigator's assessment of causality is required for each adverse event. Refer to Table 10 for instructions in evaluating adverse events. 
Table 10 Evaluating Adverse Events

\begin{tabular}{|c|c|c|}
\hline \multirow{3}{*}{$\begin{array}{l}\text { Maximum } \\
\text { Intensity }\end{array}$} & Mild & $\begin{array}{l}\text { awareness of sign or symptom, but easily tolerated (for pediatric trials, awareness of symptom, but easily tolerated) } \\
\end{array}$ \\
\hline & Moderate & discomfort enough to cause interference with usual activity (for pediatric trials, definitely acting like something is wrong) \\
\hline & Severe & incapacitating with inability to work or do usual activity (for pediatric trials, extremely distressed or unable to do usual activities) \\
\hline \multirow[t]{9}{*}{ Seriousness } & \multicolumn{2}{|c|}{ A serious adverse event (AE) is any adverse event occurring at any dose or during any use of Sponsor's product that: } \\
\hline & \multicolumn{2}{|c|}{$\dagger$ Results in death; or } \\
\hline & \multicolumn{2}{|c|}{$\begin{array}{l}\text { †Is life threatening; or places the subject, in the view of the investigator, at immediate risk of death from the event as it occurred [Note: This does not include an } \\
\text { adverse event that, had it occurred in a more severe form, might have caused death.]; or }\end{array}$} \\
\hline & \multicolumn{2}{|c|}{$†$ Results in a persistent or significant disability/incapacity (substantial disruption of one's ability to conduct normal life functions); or } \\
\hline & \multicolumn{2}{|c|}{$\begin{array}{l}\text { †Results in or prolongs an existing inpatient hospitalization (hospitalization is defined as an inpatient admission, regardless of length of stay, even if the } \\
\text { hospitalization is a precautionary measure for continued observation. (Note: Hospitalization for an elective procedure to treat a pre-existing condition that has not } \\
\text { worsened is not a serious adverse event. A pre-existing condition is a clinical condition that is diagnosed prior to the use of a Merck product and is documented in the } \\
\text { patient's medical history.); or }\end{array}$} \\
\hline & \multicolumn{2}{|c|}{$\dagger$ Is a congenital anomaly/birth defect (in offspring of subject taking the product regardless of time to diagnosis); or } \\
\hline & \multicolumn{2}{|c|}{ Is a cancer; or } \\
\hline & \multirow{2}{*}{\multicolumn{2}{|c|}{$\begin{array}{l}\text { Is associated with an overdose (whether accidental or intentional). Any adverse event associated with an overdose is considered a serious adverse event. An } \\
\text { overdose that is not associated with an adverse event is considered a non-serious event of clinical interest and must be reported within } 24 \text { hours. } \\
\text { Other important medical events that may not result in death, not be life threatening, or not require hospitalization may be considered a serious adverse event when, } \\
\text { based upon appropriate medical judgment, the event may jeopardize the subject and may require medical or surgical intervention to prevent one of the outcomes listed } \\
\text { previously (designated above by a } \dagger \text { ). }\end{array}$}} \\
\hline & & \\
\hline Duration & \multicolumn{2}{|c|}{ Record the start and stop dates of the adverse event. If less than 1 day, indicate the appropriate length of time and units } \\
\hline Action taken & \multicolumn{2}{|c|}{ Did the adverse event cause the Sponsor's product to be discontinued? } \\
\hline \multirow[t]{4}{*}{$\begin{array}{l}\text { Relationship to } \\
\text { Sponsor's } \\
\text { Product }\end{array}$} & \multicolumn{2}{|c|}{$\begin{array}{l}\text { Did the Sponsor's product cause the adverse event? The determination of the likelihood that the Sponsor's product caused the adverse event will be provided by an } \\
\text { investigator who is a qualified physician. The investigator's signed/dated initials on the source document or worksheet that supports the causality noted on the AE } \\
\text { form, ensures that a medically qualified assessment of causality was done. This initialed document must be retained for the required regulatory time frame. The } \\
\text { criteria below are intended as reference guidelines to assist the investigator in assessing the likelihood of a relationship between the test drug and the adverse event } \\
\text { based upon the available information. } \\
\text { The following components are to be used to assess the relationship between the Sponsor's product and the AE; the greater the correlation with the components } \\
\text { and their respective elements (in number and/or intensity), the more likely the Sponsor's product caused the adverse event: }\end{array}$} \\
\hline & Exposure & $\begin{array}{l}\text { Is there evidence that the subject was actually exposed to the Sponsor's product such as: reliable history, acceptable compliance assessment (pill } \\
\text { count, diary, etc.), expected pharmacologic effect, or measurement of drug/metabolite in bodily specimen? }\end{array}$ \\
\hline & Time Course & $\begin{array}{l}\text { Did the AE follow in a reasonable temporal sequence from administration of the Sponsor's product? } \\
\text { Is the time of onset of the AE compatible with a drug-induced effect (applies to trials with investigational medicinal product)? }\end{array}$ \\
\hline & Likely Cause & $\begin{array}{l}\text { Is the AE not reasonably explained by another etiology such as underlying disease, other drug(s)/vaccine(s), or other host or environmental } \\
\text { factors }\end{array}$ \\
\hline
\end{tabular}


Protocol/Amendment No.: 052-04

\begin{tabular}{|c|c|c|}
\hline \multirow{3}{*}{$\begin{array}{l}\text { Relationship } \\
\text { to Sponsor's } \\
\text { Product } \\
\text { (continued) }\end{array}$} & \multicolumn{2}{|c|}{ The following components are to be used to assess the relationship between the Sponsor's product and the AE: (continued) } \\
\hline & Dechallenge & $\begin{array}{l}\text { Was the Sponsor's product discontinued or dose/exposure/frequency reduced? } \\
\text { If yes, did the AE resolve or improve? } \\
\text { If yes, this is a positive dechallenge. If no, this is a negative dechallenge. } \\
\text { (Note: This criterion is not applicable if: (1) the AE resulted in death or permanent disability; (2) the AE resolved/improved despite } \\
\text { continuation of the Sponsor's product; (3) the trial is a single-dose drug trial); or (4) Sponsor's product(s) is/are only used one time.) }\end{array}$ \\
\hline & Rechallenge & $\begin{array}{l}\text { Was the subject re-exposed to the Sponsor's product in this trial? } \\
\text { If yes, did the AE recur or worsen? } \\
\text { If yes, this is a positive rechallenge. If no, this is a negative rechallenge. } \\
\text { (Note: This criterion is not applicable if: (1) the initial AE resulted in death or permanent disability, or (2) the trial is a single-dose drug trial); } \\
\text { or (3) Sponsor's product(s) is/are used only one time.) } \\
\text { NOTE: IF A RECHALLENGE IS PLANNED FOR AN ADVERSE EVENT WHICH WAS SERIOUS AND WHICH MAY HAVE BEEN } \\
\text { CAUSED BY THE SPONSOR'S PRODUCT, OR IF RE-EXPOSURE TO THE SPONSOR'S PRODUCT POSES ADDITIONAL POTENTIAL } \\
\text { SIGNIFICANT RISK TO THE SUBJECT THEN THE RECHALLENGE MUST BE APPROVED IN ADVANCE BY THE SPONSOR } \\
\text { CLINICAL DIRECTOR AND THE INSTITUTIONAL REVIEW BOARD/INDEPENDENT ETHICS COMMITTEE. }\end{array}$ \\
\hline & \begin{tabular}{l}
\multicolumn{2}{l}{ Consistency } \\
with Trial \\
Treatment \\
Profile \\
\end{tabular} & $\begin{array}{l}\text { Is the clinical/pathological presentation of the } \mathrm{AE} \text { consistent with previous knowledge regarding the Sponsor's product or drug class } \\
\text { pharmacology or toxicology? }\end{array}$ \\
\hline \multicolumn{3}{|c|}{$\begin{array}{l}\text { The assessment of relationship will be reported on the case report forms /worksheets by an investigator who is a qualified physician according to his/her best clinical judgment, including } \\
\text { consideration of the above elements. }\end{array}$} \\
\hline \multicolumn{2}{|c|}{ Record one of the following: } & Use the following scale of criteria as guidance (not all criteria must be present to be indicative of a Sponsor's product relationship). \\
\hline $\begin{array}{l}\text { Yes, there is } \\
\text { possibility of } S \\
\text { relationship. }\end{array}$ & $\begin{array}{cr}\text { a } & \text { reasonable } \\
\text { ponsor's } & \text { product }\end{array}$ & $\begin{array}{l}\text { There is evidence of exposure to the Sponsor's product. The temporal sequence of the AE onset relative to the administration of the Sponsor's } \\
\text { product is reasonable. The AE is more likely explained by the Sponsor's product than by another cause. }\end{array}$ \\
\hline $\begin{array}{l}\text { No, there is } n \\
\text { possibility of } S \\
\text { relationship }\end{array}$ & $\begin{array}{l}\text { ot a reasonable } \\
\text { ponsor's product }\end{array}$ & $\begin{array}{l}\text { Subject did not receive the Sponsor's product OR temporal sequence of the AE onset relative to administration of the Sponsor's product is not } \\
\text { reasonable OR there is another obvious cause of the AE. (Also entered for a subject with overdose without an associated AE.) }\end{array}$ \\
\hline
\end{tabular}




\subsubsection{Sponsor Responsibility for Reporting Adverse Events}

All Adverse Events will be reported to regulatory authorities, IRB/IECs and investigators in accordance with all applicable global laws and regulations.

\subsection{TRIAL GOVERNANCE AND OVERSIGHT}

\subsubsection{Scientific Advisory Committee}

This trial was developed in collaboration with a Scientific Advisory Committee (SAC). The SAC comprises both Sponsor and non-Sponsor scientific experts who provide input with respect to trial design, interpretation of trial results and subsequent peer-reviewed scientific publications.

\subsubsection{Data Monitoring Committee}

To supplement the routine trial monitoring outlined in this protocol, an external Data Monitoring Committee (DMC) will monitor the interim data from this trial. The voting members of the committee are external to the Sponsor. The members of the DMC must not be involved with the trial in any other way (e.g., they cannot be trial investigators) and must have no competing interests that could affect their roles with respect to the trial. The DMC will include 3-5 clinicians experienced in hepatology and nephrology and 1 external statistician; this is in addition to the unblinded trial statistician who will be a non-voting member of the committee.

The DMC will make recommendations to the SPONSOR regarding steps to ensure both subject safety and the continued ethical integrity of the trial. Also, the DMC will review interim trial results, consider the overall risk and benefit to trial participants (see Section 8.2.9 - Interim Analyses) and recommend to the SPONSOR if the trial should continue in accordance with the protocol.

Specific details regarding responsibilities and governance, including the roles and responsibilities of the various members and the SPONSOR; meeting facilitation; the trial governance structure; and requirements for and proper documentation of DMC reports, minutes, and recommendations will be described in a separate charter that is reviewed and approved by the DMC. The DMC will monitor the trial at an appropriate frequency, as described in the detailed DMC charter. The DMC will also make recommendations to the SPONSOR regarding steps to ensure both subject safety and the continued ethical integrity of the trial.

A DMC recommendation will be communicated to the Sponsor as agreed to in the Collaboration agreement.

\subsection{STATISTICAL ANALYSIS PLAN}

This section outlines the statistical analysis strategy and procedures for the study. If, after the study has begun, changes are made to primary and/or key secondary hypotheses, or the 
The primary hypothesis is that patients treated with MK-5172 + MK-8742 for 12 weeks will achieve a $S_{12}$ rate higher than the reference $S_{12}$ rate of $45 \%$. The hypothesis will be evaluated within the subjects of the immediate treatment and the intensive PK arms, and it will be tested at two-sided significant level (type-I error) of 0.05. A 95\% asymptotic (Wald) confidence interval (CI) will also be constructed for the SVR 12 rate.

Several considerations led us to choose a reference SVR of $45 \%$ for this study:

(1) IFN mono-therapy is recommended for HCV-infected patients with CKD stages 3-5 who are on or not yet on maintenance dialysis therapy [19]. The meta-analyses, conducted by Fabrizi et al, revealed a summary SVR 24 of 39\% (CI 32\%-46\%) [20].

(2) Given the substantial variation in the GT1 proportion of the studies (ranging from 0 to 1) in the Fabrizi meta-analyses, a Bayesian logistic regression model for SVR was used to account for the variation of GT1 proportions. Twenty studies with GT1 proportion were identified from the Fabrizi paper and included in the re-analysis. Non-informative priors were used for the Bayesian random-effect model containing a random intercept and a fixedeffect of GT1 proportion. The model predicts that, if the studies had enrolled 100\% GT1, the posterior probability/confidence that the true overall population mean for SVR rate would have been at most $45 \%$ is about 0.90 .

(3) A SVR of approximately 40\% was observed in a large study of PEG-IFN/RBV in 3,070 HCV GT1 patients without renal disease conducted in the United States [28]. The SVR response of patients with CKD stage 4-5 is not expected to be higher than that of the general $\mathrm{HCV}$ population without renal disease.

\subsubsection{Safety Analysis}

The All-Subjects-as-Treated population will be employed for safety analyses. For this protocol, the proportion of subjects who experience the following adverse events during the study treatment period will be estimated for each arm: adverse events of elevated laboratory values that are reported as ECIs described in section 7.2.3.2 (Tier 1 events). The Tier 1 events rates of the immediate treatment arm will be compared to those of the placebo treatment period of the deferred treatment arm. P-values and 95\% confidence intervals for between-treatment differences will be calculated using the Miettinen and Nurminen method [29].

Safety and tolerability will be carefully monitored throughout the study by the SPONSOR (or designee) in accordance with standard procedures and also by an external Data Monitoring Committee (DMC).

\subsubsection{Power and Sample Size}

This study will randomize 105 subjects into the immediate treatment arm and 105 subjects into the deferred treatment group. In addition, 10 subjects will be enrolled as intensive PK cohort. The primary hypothesis will be evaluated within the subjects of the immediate treatment and the intensive PK arms $(n=115)$. It would have at least 95\% power to 
demonstrate that the $\mathrm{SVR}_{12}$ rate of $\mathrm{MK}-5172+\mathrm{MK}-8742$ is higher than the reference $\mathrm{SVR}_{12}$ rate of $45 \%$ at an overall one-sided $0.025 \alpha$-level, if the true $S_{12}$ rate of $M K-5172+$ MK8742 is about $65 \%$. The power and sample size are based on the assumption that approximately $10 \%$ of the randomized subjects would have missing $\mathrm{SVR}_{12}$ rate due to death or early discontinuation from study with reasons unrelated to their responses to the HCV treatment and will be excluded from the mFAS population (i.e. assuming the mFAS population size of 103 subjects). The calculation is based on SAS PROC POWER based on ztest using the normal approximation to the binomial distribution.

\subsection{Statistical Analysis Plan}

\subsubsection{Responsibility for Analyses/In-House Blinding}

The statistical analyses of the data obtained from this study will be the responsibility of the Clinical Biostatistics department of the SPONSOR. Certain specific analyses such as PK, pharmacogenetics and resistance will be the responsibility of the appropriate departments of the SPONSOR.

Subjects in the immediate and deferred treatment arms, and corresponding site personnel and investigators will remain blinded to the treatment groups for the first 12 weeks of treatment period under in-house blinding procedures. The second 12 weeks of active treatment period for the deferred arm will be conducted as an open-label study. The database will be unblinded at Week 16 visit for the immediate and the deferred treatment arm. Note that the Week 16 visit takes place 16 weeks after randomization, and it is also the FU WK4 visit for the immediate treatment arm.

The Clinical Biostatistics department will generate the randomized allocation schedule(s) for study treatment assignment. Randomization will be implemented by an IVRS. (Please note, the 10 subjects assigned to the Intensive PK Arm will be open-label)

Pharmacokinetic (PK) measurements will be conducted in support of PK evaluations. Additionally, a small team as specified in a separate Modeling and Simulation (M\&S) Modeling Analysis Plan, and who are separate from the study team, will be unblinded for the purpose of preparing the pharmacokinetic analyses. No PK data or results from the PK analyses will be shared with the study team, and the unblinded group will not be members of the study team.

During the course of the trial, periodic safety analyses will be conducted. An external data monitoring committee (DMC) will be established to safeguard the interests of trial participants, to provide ongoing review of those safety data and to monitor the overall conduct of the trial.

For the first 12 weeks of treatment period, treatment-level and patient-level safety results will be provided by the unblinded statistician to DMC. Limited additional SPONSOR personnel may be unblinded to the treatment level results of these reviews, if required, in order to act on the recommendations of the DMC. The extent to which individuals are unblinded with 
respect to results of interim reviews will be documented by the unblinded statistician. The unblinded statistician will not be involved in any discussions regarding modifications to the protocol, statistical methods, identification of protocol violators, or data validation efforts after the interim analyses.

The DMC will perform periodic reviews of safety data in order to protect subject welfare and preserve study integrity. There will be no enrollment pause in the study during these reviews. The DMC is to recommend to the SPONSOR whether the nature, frequency, and severity of adverse effects associated with study treatment warrant the early termination of the study in the best interests of the participants, whether the study should continue as planned, or the study should continue with modifications. While the DMC will be asked to advise SPONSOR regarding future conduct of the study, including possible early study termination, SPONSOR retains final decision-making authority on all aspects of the study. Additional logistical details will be provided in the external DMC Charter.

\subsubsection{Hypotheses/Estimation}

Objectives and hypotheses of the study are stated in Section 3.0.

\subsubsection{Analysis Endpoints}

Efficacy and safety endpoints that will be evaluated are listed in the following sections.

\subsubsection{Efficacy/Pharmacokinetic Endpoints}

\subsection{Efficacy Endpoints}

An initial description of efficacy measures is provided in Section 4.2.3.1.

The primary efficacy endpoint will be the $\mathrm{SVR}_{12}$ rate of the subjects in the immediate treatment and the intensive PK arms.

The secondary efficacy endpoints are

1. The $\mathrm{SVR}_{4}$ and $\mathrm{SVR}_{24}$ rates of the subjects within the immediate treatment and the intensive PK arms.

2. The $\mathrm{SVR}_{4}, \mathrm{SVR}_{12}$, and $\mathrm{SVR}_{24}$ rates in the deferred treatment arm following the end of all active study therapy.

3. The $\mathrm{SVR}_{4}, \mathrm{SVR}_{12}$, and $\mathrm{SVR}_{24}$ rates following the end of all active study therapy for all treatment arms combined.

4. The emergence of viral resistant to MK-5172 and MK-8742 when administered as a combination regimen

5. Proportion of achieving TND, TD(u), and TD(q) at EOT

\subsection{Pharmacokinetic Endpoints}

An initial description of efficacy measures is provided in Section 4.2.3.3. 
Protocol/Amendment No.: 052-04

The PK endpoints for MK-5172, MK-8742 are $\mathrm{AUC}_{0-24}, \mathrm{C}_{2 \mathrm{hr}}$ and $\mathrm{C}_{\text {trough }}$

\subsection{Exploratory Endpoints}

1. The level of biomarkers (e.g., proteins and metabolite production), that may be predictive of tolerability of study drugs and virologic response to MK-5172 in combination with MK-8742

2. Change from baseline in health-related quality of life for each of the SF-36v2 eight health domain scores (Physical Functioning, Role Limitations-Physical, Bodily Pain, General Health, Vitality, Social Functioning, Role Limitations-Emotional, and Mental Health), and the Physical Component Summary (PCS) and Mental Component Summary (MCS) score

3. Change from baseline in Serum cryoglobulin level, rheumatoid factor, and C4 Complement in subjects with Cryoglobulinemia

\subsubsection{Safety Endpoints}

An initial description of safety measures is provided in Section 4.2.3.2.

The analysis of safety results will follow a tiered approach. The tiers differ with respect to the analyses that will be performed. Safety parameters or adverse events of special interest that are identified a priori constitute Tier 1 safety endpoints that will be subject to inferential testing for statistical significance with p-values and 95\% confidence intervals provided for between-group comparisons. Other safety parameters will be considered Tier 2 or Tier 3. Tier 2 parameters (requires that at least 4 subjects in each treatment group exhibit the event) will be assessed via point estimates with 95\% confidence intervals provided for betweengroup comparisons; only point estimates by treatment group are provided for Tier 3 safety parameters.

For this protocol, the Tier 1 safety parameters and adverse events are the proportion of subjects with adverse events of the following types at any time during the study therapy period: adverse events of elevated laboratory values that are reported as ECIs described in section 7.2.3.2.

The following are Tier 2 safety parameters and adverse events:

1. Proportion of subjects with adverse experiences of the following types at any time during the study therapy period: (1) at least one adverse event; (2) a drug-related adverse event; (3) a serious adverse event; (4) a renal serious adverse event; (5) a serious and drugrelated adverse event; (6) an adverse event leading to discontinuation from treatment; and (7) renal disease progression (defined as increasing dialysis frequency in subjects who were on hemodialysis at baseline, initiation of maintenance hemodialysis in subjects who were not on hemodialysis at baseline, or progressive increase in CKD stages). 
Protocol/Amendment No.: 052-04

2. Change from baseline in serum creatinine and BUN, estimated GFR at the end of active study therapy for the immediate treatment arm and at the end of placebo treatment for the deferred treatment arm for CKD 4-5 subjects who are not receiving HD at baseline.

Serious adverse experiences will continue to be collected throughout the study.

For all subjects in the deferred treatment arm, safety results during the period of receiving active study therapy will be summarized and described.

\subsubsection{Analysis Populations}

\subsubsection{Efficacy Analysis Populations}

The Modified Full Analysis Set (mFAS) population will serve as the primary population for the analysis of efficacy data in this study. The mFAS population is a subset of the subjects, who are randomized to the immediate treatment arm or who are assigned to the intensive PK arm, with subjects excluded for the following reasons:

- failure to receive at least one dose of study treatment

- missing data due to death with reasons unrelated to study drug or reasons other than liver disease

- missing data due to study discontinuation with reasons unrelated to progression of liver disease, study drug and their responses to the HCV treatment.

All subjects who return for follow-up visits will be included in the corresponding SVR analysis, regardless of the length of treatment received or reason for discontinuation.

A supportive analysis using the Per-Protocol (PP) population will be performed for the primary $\left(\mathrm{SVR}_{12}\right)$ and key secondary efficacy endpoints $\left(\mathrm{SVR}_{4}\right.$ and $\left.\mathrm{SVR}_{24}\right)$. The PP population is a subset of the mFAS population. The PP population excludes subjects due to important deviations from the protocol that may substantially affect the results of the primary and key secondary efficacy endpoints. Potential violations that may result in the exclusion of a subject from the PP population include:

- Violations of specific inclusion/exclusion criteria:

- The subject is infected with a non-GT $1 \mathrm{HCV}$ infection at entry or during the course of the study, including a mixed GT infection (with a non-GT 1) or a nontypeable genotype

- The subject met criteria for futility, virologic breakthrough or end of treatment failure but had undetectable MK-5172 levels at one or more pharmacokinetic sampling timepoints temporally associated with the failure timepoint

- The subject received concomitant medications that are prohibited due to their potential to result in a clinically significant lowering of the MK-5172 concentrations including: 
- $\quad$ CYP3A4 inducers such as rifampin, carbamazepine and efavirenz

- $\quad$ P-gp inducers such as St. John's Wort

- Any co-administered medication, currently unidentified, but for which subsequent clinical DDI data indicate that co-administration with MK-5172 leads to a clinically significant lowering of MK-5172 concentrations

- Other violations may be identified during the course of data collection and they will be listed specifically in the CSR

A subject with important deviations from the protocol as described above at randomization will be excluded from the PP population. For subjects with important deviations from the protocol as described above during course of the treatment, data obtained subsequent to the violation will be excluded from analysis.

The FAS population consists of all randomized subjects who have received at least one dose of study treatment. This is one of the supportive analysis populations in this study.

Subjects will be included in the treatment group to which they are randomized for the analysis of efficacy data using mFAS, FAS, and PP populations. Details on the approach to handling missing data are provided in Section 8.2.5 Statistical Methods.

\subsubsection{Safety Analysis Populations}

The All Subjects as Treated (ASaT) population will be used for the analysis of safety data in this study. The ASaT population consists of all randomized subjects who received at least one dose of study treatment. Subjects will be included in the treatment group corresponding to the study treatment they actually received for the analysis of safety data using the ASaT population. For most subjects this will be the treatment group to which they are randomized. Subjects who take incorrect study treatment for the entire treatment period will be included in the treatment group corresponding to the study treatment they actually received.

At least one laboratory or vital sign measurement obtained subsequent to at least one dose of study treatment is required for inclusion in the analysis of each specific parameter. To assess change from baseline, a baseline measurement is also required.

Details on the approach to handling missing data for safety analyses are provided in Section 8.2.5 Statistical Methods.

\subsubsection{Statistical Methods}

The approach to handling missing data is described in Section 8.2.5.1. Statistical testing and inference for safety analyses are described in Section 8.2.5.2. Unless otherwise stated, all statistical tests will be conducted at the $\alpha=0.05$ (2-sided) level. 


\subsubsection{Statistical Methods for Efficacy Analyses}

\section{Missing values}

A missing data point for a given study visit may be due to any one of the following reasons: a visit occurred but data were not collected or were unusable; a visit did not occur; and a subject discontinued from the study before reaching the visit. Subjects who prematurely discontinued the assigned treatment should remain in the study for the follow-up, if possible.

The HCV RNA outcome is categorized as TND, TD(u), and TD(q). There are 3 types of missing data handled by different approaches.

1. Intermittent missing: If a missing data point is immediately preceded and followed by non-missing HCV RNA outcomes, the missing value would be imputed to the worse outcome of the two. For example, if a missing data point is preceded by $\operatorname{TD}(q)$ and followed by $\mathrm{TD}(\mathrm{u})$ or $\mathrm{TND}$, then the missing value would be imputed as $\mathrm{TD}(\mathrm{q})$; if a missing data point is preceded by $\mathrm{TD}(\mathrm{u})$ and followed by TND, then the missing value would be imputed as TD(u); when a missing value is flanked by two TND, then the missing value would be imputed as TND.

2. Non-intermittent missing related to the study drug: For missing values due to death with reasons related to study drug or liver disease, and missing values due to premature study discontinuations with treatment related reasons such as, progression of liver disease, clinical or laboratory adverse event related to the study drug, or lack of efficacy (e.g. discontinuation from the study following a confirmed HCV RNA TD(q)), the missing values will be considered as treatment failures and thereafter.

3. Non-intermittent missing unrelated to the study drug: For missing data due to death with reasons unrelated to study drug or reasons other than liver disease, and due to premature study discontinuations with reasons unrelated to treatment such as loss to follow-up, protocol violation, patient withdrew consent, etc., the missingness mechanism is unlikely to related to patients' response to the HCV treatment, and therefore the missing at random (MAR) assumption is plausible. The approaches to address this type of missing data depend on the analytical strategy, and they are described in the following sections.

In addition, a missing baseline/Day1 HCV RNA result will be replaced with a screening result, if available. Missing values in the health-related quality of life data will not be imputed.

\section{$\underline{S V R}_{12}$ rate (proportion of achieving SVR $\underline{12}$ )}

The study hypothesis is that patients treated with MK-5172 + MK-8742 for 12 weeks will achieve a SVR12 rate higher than the reference SVR12 rate of $45 \%$. Correspondingly, the null hypothesis $\left(\mathrm{H}_{0}\right)$ is that $\mathrm{SVR}_{12}$ rate $\leq 45 \%$; the alternative hypothesis $(\mathrm{Ha})$ is that $\mathrm{SVR}_{12}$ rate $>45 \%$. A treatment effect is established statistically by showing that the lower bound of the two-sided $95 \%$ confidence interval for $\mathrm{SVR}_{12}$ rate $>45 \%$. A Wald test will be performed 
Protocol/Amendment No.: 052-04

at two-sided significant level (type-I error) of 0.05 . This hypothesis will be evaluated primarily in the combined immediate treatment and intensive PK arms.

\section{$\underline{\text { Primary approach }}$}

The $\mathrm{SVR}_{12}$ rate is estimated by the ratio of the number of subjects achieving $\mathrm{SVR}_{12}$ versus the number of subjects in the mFAS population, that is, the proportion of subjects with $\mathrm{SVR}_{12}$ in the mFAS. A two-sided 95\% asymptotic (Wald) confidence interval will be calculated. Under the assumption of missing completely at random (MCAR), the mFAS population excludes subjects who did not experience virologic failure, but prematurely withdraw from study due to reasons not related to the study drug (i.e., the subjects with the type 3 missing value) . Note that subjects with documented virologic failure during the treatment or followup period, even if they withdrew prematurely due to reasons not related to study drug, are included in the mFAS population and classified as failures.

\section{Secondary approaches}

The $\mathrm{SVR}_{12}$ rate will also be estimated in the PP population as supportive analysis, using the approach described above.

Sensitivity analysis will be conducted in the FAS population to address the robustness of the study conclusion to the management of the type 3 missing information. In this analysis, the type 3 missing will be imputed as failure. The $\mathrm{SVR}_{12}$ rate is estimated by the ratio of the number of subjects achieving $\mathrm{SVR}_{12}$ versus the number of subjects in the FAS population, that is, the proportion of subjects with $\mathrm{SVR}_{12}$ in the FAS. However, it is useful to note that this approach invokes maximal stress to the robustness of the study results and puts a bound on the extent of the impact of the type 3 missing information.

\section{Other efficacy endpoints}

The EOT HCV RNA for the immediate treatment and intensive PK arms and for the deferred treatment arm will be summarized as proportion according to the categories of TND, TD(u), and $\operatorname{TD}(q)$. Table 12 summarizes the key efficacy analyses. 
Protocol/Amendment No.: 052-04

Table 12 Analysis Strategy for Efficacy Endpoints

\begin{tabular}{|c|c|c|c|c|}
\hline $\begin{array}{c}\text { Endpoint/Variable } \\
\text { (Description, Time point) }\end{array}$ & $\begin{array}{l}\text { Primary vs } \\
\text { Secondary } \\
\text { Approach }^{\dagger}\end{array}$ & Statistical Method & $\begin{array}{c}\text { Analysis } \\
\text { Population }\end{array}$ & $\begin{array}{l}\text { Approach for } \\
\text { Non-Intermittent } \\
\text { Missing Data }\end{array}$ \\
\hline \multicolumn{5}{|l|}{ Primary: } \\
\hline $\mathrm{SVR}_{12}$ rate & $\mathrm{P}$ & $\begin{array}{c}\text { Wald test } \\
\text { (95\% asymptotic CI) }\end{array}$ & mFAS & $\begin{array}{c}\mathrm{TRD}=\mathrm{F}^{\ddagger} \\
\text { (i.e., } \mathrm{M}=\mathrm{F}^{\ddagger} \text { for type } 2 \\
\mathrm{DAO}^{\ddagger} \text { for type } 3\end{array}$ \\
\hline $\mathrm{SVR}_{12}$ rate & $\mathrm{S}$ & $\begin{array}{c}\text { Wald test } \\
(95 \% \text { asymptotic CI) }\end{array}$ & FAS & $\begin{array}{c}\mathrm{M}=\mathrm{F} \text { for type } 2 \text { and } \\
\text { type } 3\end{array}$ \\
\hline $\mathrm{SVR}_{12}$ rate & $\mathrm{S}$ & $\begin{array}{c}\text { Wald test } \\
\text { (95\% asymptotic CI) }\end{array}$ & $\mathrm{PP}$ & $\begin{array}{l}\text { TRD }=\mathrm{F} \\
\text { (i.e., } \mathrm{M}=\mathrm{F} \text { for type } 2 \\
\text { DAO for type } 3 \text { ) }\end{array}$ \\
\hline \multicolumn{5}{|l|}{ Secondary } \\
\hline $\mathrm{SVR}_{4}$ and $\mathrm{SVR}_{24}$ rates & $\mathrm{P}$ & 95\% asymptotic CI & mFAS & $\begin{array}{l}\text { TRD }=\mathrm{F} \\
\text { (i.e., } \mathrm{M}=\mathrm{F} \text { for type } 2 \\
\text { DAO for type } 3\end{array}$ \\
\hline $\mathrm{SVR}_{4}$ and $\mathrm{SVR}_{24}$ rates & $S$ & 95\% asymptotic CI & FAS & $\begin{array}{c}\mathrm{M}=\mathrm{F} \text { for type } 2 \text { and } \\
\text { type } 3\end{array}$ \\
\hline $\mathrm{SVR}_{4}$ and $\mathrm{SVR}_{24}$ rates & $S$ & 95\% asymptotic CI & $\mathrm{PP}$ & $\begin{array}{l}\text { TRD }=\mathrm{F} \\
\text { (i.e., } \mathrm{M}=\mathrm{F} \text { for type } 2 \\
\text { DAO for type } 3\end{array}$ \\
\hline \multicolumn{5}{|c|}{$\begin{array}{l}\text { P=Primary approach; } \mathrm{S}=\text { Secondary approach. } \\
\text { Imputation for specific missing values described in Section 8.2.5.1 } \\
\text { TRD=F is Treatment-Related Discontinuation = Failure; DAO = Dat }\end{array}$} \\
\hline
\end{tabular}

\section{Subject Virologic Failure: Non-response, Rebound, Breakthrough, and Relapse}

Summary statistics will be provided to describe the rates of occurrence of subject virologic non-response, rebound, breakthrough, and relapse. Definitions for subject virologic nonresponse, rebound, breakthrough, and relapse are in Section 4.2.3.1.1.2

\subsubsection{Statistical Methods for Safety Analyses}

Safety and tolerability will be assessed by clinical review of all relevant parameters including ECIs, adverse events and laboratory parameters.

The analysis of safety results will follow a tiered approach (Table 13). The tiers differ with respect to the analyses that will be performed. Safety parameters or adverse experiences of special interest that are identified a priori constitute "Tier 1" safety endpoints that will be subject to inferential testing for statistical significance with p-values and $95 \%$ confidence intervals provided for between-group comparisons. Other safety parameters will be considered Tier 2 or Tier 3. Tier 2 parameters will be assessed via point estimates with 95\% confidence intervals provided for between-group comparisons; only point estimates by treatment group are provided for Tier 3 safety parameters.

Adverse experiences (specific terms as well as system organ class terms) and predefined limits of change in laboratory and vital signs that are not pre-specified as endpoints of special 
interest will be classified as belonging to "Tier 2" or "Tier 3", based on the number of events observed. Membership in Tier 2 requires that at least 4 patients in any treatment group exhibit the event; all other adverse experiences and predefined limits of change will belong to Tier 3.

The threshold of at least 4 events was chosen because the 95\% confidence interval for the between-group difference in percent incidence will always include zero when treatment groups of equal size each have less than 4 events and thus would add little to the interpretation of potentially meaningful differences. Because many $95 \%$ confidence intervals may be provided without adjustment for multiplicity, the confidence intervals should be regarded as a helpful descriptive measure to be used in review, not a formal method for assessing the statistical significance of the between-group differences in adverse experiences and predefined limits of change.

Changes from baseline in laboratory and vital signs that are not pre-specified as endpoints of special interest will be considered Tier 3 safety parameters. Summary statistics for baseline, on-treatment, and change from baseline values will be provided in table format.

Values for missing safety laboratory data or missing vital signs will not be imputed; however, a missing Baseline/Day 1 result will be replaced with a screening result, if available. If no pretreatment laboratory value is available, the Baseline/Day 1 value will be assumed to be normal (i.e., no grade [Grade 0]) for the summary of graded laboratory abnormalities. Missing values will be handled using the Data-As-Observed (DAO) approach.

For this protocol, the primary safety analysis will compare the safety data in the immediate treatment arm during the treatment period to those of the deferred treatment arm during the placebo treatment period. For categorical data, p-values (Tier 1 only) and 95\% confidence intervals (Tier 1 and Tier 2) will be provided for between-treatment differences in the percentage of patients with events; these analyses will be performed using the Miettinen and Nurminen method [29], an unconditional, asymptotic method. For continuous data (as Tier 2), 95\% confidence intervals will be provided for differences in the group means using asymptotic method

The safety parameters and adverse events during the active study therapy period, or within 14 days after discontinuing active study therapy will also be summarized for the combined immediate treatment and intensive PK arms, and for the differed treatment arm. 95\% CI will be provided for the proportion of subjects with the Tier 1 and Tier 2 adverse events. 
Protocol/Amendment No.: 052-04

Table 13 Analysis Strategy for Safety Parameters

\begin{tabular}{|c|c|c|c|c|}
\hline Safety Tier & Safety Endpoint ${ }^{\dagger}$ & p-Value & $\begin{array}{c}\text { 95\% CI for } \\
\text { Treatment } \\
\text { Comparison }\end{array}$ & $\begin{array}{l}\text { Descriptive } \\
\text { Statistics }\end{array}$ \\
\hline Tier 1 & $\begin{array}{l}\text { AEs of elevated laboratory values that are } \\
\text { reported as ECIs }\end{array}$ & $\mathrm{X}$ & $\mathrm{X}$ & $\mathrm{X}$ \\
\hline Tier 2 & $\begin{array}{l}\text { Any AE } \\
\text { Any Serious AE } \\
\text { Any Drug-Related AE } \\
\text { Any Serious and Drug-Related AE } \\
\text { Renal SAE } \\
\text { Discontinuation due to AE } \\
\text { Renal disease progression } \\
\text { Change from Baseline Results (serum } \\
\text { creatinine, BUN, and estimated GFR) } \\
\text { Specific AEs, SOCs, or PDLCs }{ }^{\ddagger} \text { (incidence } \geq 4 \\
\text { of patients in one of the treatment groups) }\end{array}$ & & $\begin{array}{l}\text { X } \\
X \\
X \\
X \\
X \\
X \\
X \\
X \\
X\end{array}$ & $\begin{array}{l}\text { X } \\
X \\
X \\
X \\
X \\
X \\
X \\
X \\
X\end{array}$ \\
\hline Tier 3 & $\begin{array}{l}\text { Specific AEs, SOCs, or PDLCs }{ }^{\mp} \text { (incidence }<4 \\
\text { of patients in one of the treatment groups) } \\
\text { Change from Baseline Results (Labs and Vital } \\
\text { Signs) }\end{array}$ & & & $\begin{array}{l}\mathrm{X} \\
\mathrm{X}\end{array}$ \\
\hline \multicolumn{5}{|c|}{$\begin{array}{l}\text { Adverse events references refer to both Clinical and Laboratory AEs. }^{+} \\
\text {Includes only those endpoints not pre-specified as Tier } 1 \text { or not already pre-specifed as Tier-2 } \\
\text { endpoints. } \\
\text { Note: SOC=System Organ Class; PDLC=Pre-Defined Limit of Change; } \mathrm{X}=\text { results will be provided. }\end{array}$} \\
\hline
\end{tabular}

\subsubsection{Summaries of Baseline Characteristics, Demographics, and Other Analyses Demographic and Baseline Characteristics}

The comparability of the treatment groups for each relevant characteristic will be assessed by the use of descriptive statistics. No statistical hypothesis tests will be performed on these characteristics. The number and percentage of subjects screened, randomized, the primary reasons for screen failure, and the primary reason for discontinuation will be displayed. Demographic variables (e.g., age, gender, and genotype subtype), primary and secondary diagnoses, prior and concomitant therapies will be summarized by treatment arm using descriptive statistics for continuous or categorical variables, as appropriate. Summary statistics for the baseline efficacy measure (HCV RNA) will also be provided by treatment group.

\section{Pharmacokinetic Analyses}

Summary statistics for the concentrations of MK-5172 and MK-8742 will be provided for the immediate treatment and the intensive PK groups. PK/PD analysis may also be performed within the intensive PK and evening pre-dose PK cohorts for MK-5172 and MK-8742.

\section{Viral Resistance Measurements}


Viral resistance testing will focus on the entire NS3/4A and NS5A regions for all subjects and for those who meet the subject virologic failure criteria (see Section 4.2.3.1.).

HCV genotyping is conducted using the Versant HCV genotype (LiPA) 2.0 manufactured by Innogenetics. In the US, the assay is distributed by Siemens.

\section{$\underline{\text { IL28B Analyses and Other Genetic Analysis }}$}

Exploratory descriptive analyses will include demographic and selected baseline characteristics by $I L 28 B$ genotype overall, as well as SVR 12 by IL28B genotype by treatment arm. Additional genetic analysis may be conducted to identify variations in HLA and ADME genes related to liver injury or other safety findings.

\section{$\underline{\text { Patient-Reported Outcomes Measurement }}$}

Descriptive summary statistics will be provided for the change from baseline scores for each of the SF-36v2 eight health domains (Physical Functioning, Role Limitations-Physical, Bodily Pain, General Health, Vitality, Social Functioning, Role Limitations-Emotional, and Mental Health), and Physical Component Summary (PCS) and Mental Component Summary (MCS). These analyses will be conducted for the immediate and deferred treatment arms at Week 12, Week 28 (deferred treatment arm only), Follow-Up Week 12, and Early Discontinuation. Missing data will not be imputed and the analysis will be based on observed data only (DAO approach). These analyses will be based on the FAS population. No multiplicity adjustment will be applied.

\subsubsection{Multiplicity}

As there is only a single primary efficacy hypothesis which is being conducted at the onesided $\alpha=0.025$ level, no multiplicity adjustment is needed for the primary efficacy analysis. The secondary efficacy objectives are estimation objectives, are supportive in nature and have no associated hypotheses. Therefore, no multiplicity adjustment is necessary for the secondary efficacy analysis.

\subsubsection{Sample Size and Power Calculations}

\subsubsection{Efficacy Analysis}

This study will randomize 105 subjects into the immediate treatment arm and 105 subjects into the deferred treatment group. In addition, 10 subjects will be enrolled as intensive PK cohort. The primary hypothesis will be evaluated within the subjects of the immediate treatment and the intensive PK arms $(n=115)$. It would have at least $95 \%$ power to demonstrate that the $\mathrm{SVR}_{12}$ rate of $\mathrm{MK}-5172+\mathrm{MK}-8742$ is higher than the reference $\mathrm{SVR}_{12}$ rate of $45 \%$ at an overall one-sided $0.025 \alpha$-level, if the true $S R_{12}$ rate of $M K-5172+$ MK8742 is about $65 \%$. The power and sample size are based on the assumption that approximately $10 \%$ of the randomized subjects would have missing $\mathrm{SVR}_{12}$ rate due to death or early discontinuation from study with reasons unrelated to their responses to the HCV treatment and will be excluded from the mFAS population (i.e. assuming the mFAS 
population size of 103 subjects). The calculation is based on SAS PROC POWER based on ztest using the normal approximation to the binomial distribution. Table 14 summarizes such power calculations for the primary efficacy analysis under various assumptions about the true SVR12 rate of MK-5172 + MK-8742.

Table 14 Power Calculations for the Primary Hypothesis Test Within the Subjects of the Immediate Treatment and the Intensive PK Arms

\begin{tabular}{|c|c|}
\hline \hline True $\mathrm{SVR}_{12}$ rate & Power to reject $\mathrm{H}_{0}$ \\
\hline $60 \%$ & $86 \%$ \\
\hline $62 \%$ & $93 \%$ \\
\hline $65 \%$ & $98 \%$ \\
\hline $70 \%$ & $>99 \%$ \\
\hline
\end{tabular}

\subsubsection{Safety Analysis}

The primary safety analysis will compare the safety data in the immediate treatment arm during the treatment period to those of the deferred treatment arm during the placebo period. Table 15 summarizes the power to detect an adverse event rate difference between the treatment arms using a 2-sided 5\% alpha level, if the immediate treatment arm has a two-fold or three-fold increment in adverse event rate. These calculations are under various assumptions about the true adverse event rate in the deferred treatment arm, and assume 105 subjects in each arm.

Table 16 gives the power to rule out a $50 \%$ or $100 \%$ higher adverse event rate in the immediate treatment arm at a 1-sided 2.5\% alpha level, if the event rates are the same in both the immediate and deferred treatment arms. The calculations assume 105 subjects in each arm and are based on an asymptotic method proposed by Farrington and Manning (1990).

Table 15 Power to Detect Difference in Adverse Event Rate

\begin{tabular}{|c|c|c|}
\hline $\begin{array}{c}\text { True event rate in } \\
\text { the deferred arm while } \\
\text { placebo administered }\end{array}$ & $\begin{array}{c}\text { True event rate in } \\
\text { The Immediate treatment } \\
\text { arm }\end{array}$ & $\begin{array}{c}\text { Power to } \\
\text { detect difference } \\
\text { at a 2-sided 0.05 } \alpha \text {-level) }\end{array}$ \\
\hline Two-fold increment & \multicolumn{2}{|c|}{} \\
\hline $10 \%$ & $20 \%$ & $53 \%$ \\
\hline $15 \%$ & $30 \%$ & $74 \%$ \\
\hline $20 \%$ & $40 \%$ & $89 \%$ \\
\hline $25 \%$ & $50 \%$ & $97 \%$ \\
\hline Three-fold increment & $30 \%$ & $96 \%$ \\
\hline $10 \%$ & $45 \%$ & $99 \%$ \\
\hline $15 \%$ & $60 \%$ & $>99 \%$ \\
\hline $20 \%$ & $75 \%$ & $>99 \%$ \\
\hline \hline
\end{tabular}


Protocol/Amendment No.: 052-04

Table 16 Power to Rule Out a $50 \%$ or $100 \%$ Higher Adverse Event Rate in the Immediate Treatment Arm, Assuming Both Arms Have the Same Event Rate

\begin{tabular}{|c|c|c|}
\hline $\begin{array}{c}\text { True event rate in both } \\
\text { immediate and deferred arms }\end{array}$ & $\begin{array}{c}\text { Power to rule out a 50\% } \\
\text { increase in the immediate } \\
\text { treatment arm }\end{array}$ & $\begin{array}{c}\text { Power to rule out a 100\% } \\
\text { increase in the immediate } \\
\text { treatment arm }\end{array}$ \\
\hline $10 \%$ & $21 \%$ & $63 \%$ \\
\hline $15 \%$ & $32 \%$ & $84 \%$ \\
\hline $20 \%$ & $43 \%$ & $95 \%$ \\
\hline $25 \%$ & $55 \%$ & $99 \%$ \\
\hline $30 \%$ & $66 \%$ & $>99 \%$ \\
\hline
\end{tabular}

\subsubsection{Subgroup Analyses and Effects of Baseline Factors}

To determine whether the response is consistent across various subgroups, the SVR12 rate with 95\% CIs will be estimated within each category of the following classification variables:

- Sex (female, male)

- GT: (1a vs 1 non-a)

- IL28B CC genotype vs. non-CC genotype

- $\mathrm{HCV}$ RNA at baseline, low ( $\leq 800,000 \mathrm{IU} / \mathrm{mL})$ versus high $(>800,000 \mathrm{IU} / \mathrm{mL})$

- Stage of fibrosis (Non-cirrhotic vs. Cirrhotic)

- Dialysis (yes vs. no)

- Diabetes (yes vs. no)

- CKD stage (4 and 5)

- Prior IFN or PEG-IFN \pm Ribavirin treatment response (Treatment naïve, Relapser, Partial responder, and Null Responder)

The consistency of the efficacy response will be assessed descriptively using summary statistics for each category of the classification variables listed above.

In addition, Safety comparison between the immediate and differed treatment arms will also be conducted according to the subgroups of on dialysis vs. not on dialysis, diabetics vs. nondiabetics, and CKD stages (4 and 5). 


\subsubsection{Interim Analyses}

No formal interim analyses are planned for this study. During the course of the trial, periodic safety analyses will be conducted for the accruing data and will be reviewed by an external $\mathrm{DMC}$ at regular intervals to ensure the safety of the patients participating in the clinical trial.

\subsubsection{Compliance (Medical Adherence)}

In this study, as part of the routine recording of the amount of study treatment taken by each subject, the number of tablets remaining in study packaging will be counted, reviewed, and recorded at regular intervals. These results will be used to calculate subject compliance.

A day within the study will be considered an "On-Therapy" day if the subject takes the MK5172 100mg/MK-8742 50mg or the Placebo tablet(s). The "Number of Days Should be on Therapy" is the total number of days from randomization to the date of the last dose of study medication for that subject. Note, the date of the last dose of study medication would be the last scheduled day for treatment administration for subject who completed the assigned treatment.

For each subject, percent compliance will then be calculated using the following formula:

$$
\text { Percent Compliance }=\frac{\text { Number of Days on Therapy }}{\text { Number of Days Should be on Therapy }} \times 100 \text {. }
$$

Summary statistics will be provided on percent compliance.

\subsection{LABELING, PACKAGING, STORAGE AND RETURN OF CLINICAL SUPPLIES}

\subsection{Investigational Product}

The investigator shall take responsibility for and shall take all steps to maintain appropriate records and ensure appropriate supply, storage, handling, distribution and usage of investigational product in accordance with the protocol and any applicable laws and regulations.

Clinical Supplies will be provided by the Sponsor as summarized in Table 17.

Clinical supplies will be packaged to support enrollment and replacement subjects as required. When a replacement subject is required, the Sponsor or designee needs to be contacted prior to dosing the replacement supplies. 
Protocol/Amendment No.: 052-04

Table 17 Product Descriptions

\begin{tabular}{|c|c|}
\hline Product Name \& Potency & Dosage Form \\
\hline MK-5172 100mg & Tablet \\
\hline MK-5172 0mg & Tablet \\
\hline MK-8742 50mg & Tablet \\
\hline MK-8742 0mg & Tablet \\
\hline MK-5172 100mg / MK8742 50mg & Tablet \\
\hline MK-5172 0mg / MK8742 0mg & Tablet \\
\hline
\end{tabular}

All placebos were created by the Sponsor to match the active product.

The single entity tablet formulations will be used in the Intensive PK arm and for the first 12 weeks of dosing in the Immediate Treatment and Deferred Treatment arms. The fixed dose combination formulation will be used for Week 16 to Week 28 dosing in the Deferred Treatment arm.

\subsection{Packaging and Labeling Information}

Clinical supplies will be affixed with a clinical label in accordance with regulatory requirements.

Subjects will receive open label and blinded monthly finished good bottles. No kitting is required.

\subsection{Clinical Supplies Disclosure}

Part of the trial is open-label; therefore, the subject, the trial site personnel, the Sponsor and/or designee are not blinded. Treatment (name, strength or potency) is included in the label text; random code/disclosure envelopes or lists are not provided.

The central electronic randomization system (IVRS/IWRS) should be used in order to unblind subjects and to unmask treatment identity. The Sponsor will not provide random code/disclosure envelopes or lists with the clinical supplies.

Treatment identification information is to be unmasked ONLY if necessary for the welfare of the subject. Every effort should be made not to unblind the subject unless necessary.

In the event that unblinding has occurred, the circumstances around the unblinding (e.g., date and reason) must be documented promptly, and the Sponsor Clinical Director notified as soon as possible. Only the principal investigator or delegate and the respective subject's code should be unblinded. Trial site personnel and Sponsor personnel directly associated with the conduct of the trial should not be unblinded. 


\subsection{Storage and Handling Requirements}

Clinical supplies must be stored in a secure, limited-access location under the storage conditions specified on the label.

Receipt and dispensing of trial medication must be recorded by an authorized person at the trial site.

Clinical supplies may not be used for any purpose other than that stated in the protocol.

\subsection{Returns and Reconciliation}

The investigator is responsible for keeping accurate records of the clinical supplies received from the Sponsor or designee, the amount dispensed to and returned by the subjects and the amount remaining at the conclusion of the trial.

For all trial sites, the local country Sponsor personnel or designee will provide appropriate documentation that must be completed for drug accountability and return.

\subsection{Standard Policies}

Trial site personnel will have access to a central electronic randomization system (IVRS/IWRS system) to allocate subjects, to assign treatment to subjects and to manage the distribution of clinical supplies. Each person accessing the IVRS system must be assigned an individual unique PIN. They must use only their assigned PIN to access the system, and they must not share their assigned PIN with anyone.

\subsection{ADMINISTRATIVE AND REGULATORY DETAILS}

\subsection{Confidentiality}

\subsubsection{Confidentiality of Data}

By signing this protocol, the investigator affirms to the Sponsor that information furnished to the investigator by the Sponsor will be maintained in confidence, and such information will be divulged to the institutional review board, ethics review committee (IRB/ERC) or similar or expert committee; affiliated institution and employees, only under an appropriate understanding of confidentiality with such board or committee, affiliated institution and employees. Data generated by this trial will be considered confidential by the investigator, except to the extent that it is included in a publication as provided in the Publications section of this protocol.

\subsubsection{Confidentiality of Subject Records}

By signing this protocol, the investigator agrees that the Sponsor (or Sponsor representative), IRB/ERC, or regulatory authority representatives may consult and/or copy trial documents in order to verify worksheet/case report form data. By signing the consent form, the subject agrees to this process. If trial documents will be photocopied during the process of verifying 
worksheet/case report form information, the subject will be identified by unique code only; full names/initials will be masked prior to transmission to the Sponsor.

By signing this protocol, the investigator agrees to treat all subject data used and disclosed in connection with this trial in accordance with all applicable privacy laws, rules and regulations.

\subsubsection{Confidentiality of Investigator Information}

By signing this protocol, the investigator recognizes that certain personal identifying information with respect to the investigator, and all subinvestigators and trial site personnel, may be used and disclosed for trial management purposes, as part of a regulatory submissions, and as required by law. This information may include:

1. name, address, telephone number and e-mail address;

2. hospital or clinic address and telephone number;

3. curriculum vitae or other summary of qualifications and credentials; and

4. other professional documentation.

Consistent with the purposes described above, this information may be transmitted to the Sponsor, and subsidiaries, affiliates and agents of the Sponsor, in your country and other countries, including countries that do not have laws protecting such information. Additionally, the investigator's name and business contact information may be included when reporting certain serious adverse events to regulatory authorities or to other investigators. By signing this protocol, the investigator expressly consents to these uses and disclosures.

If this is a multicenter trial, in order to facilitate contact between investigators, the Sponsor may share an investigator's name and contact information with other participating investigators upon request.

\subsubsection{Confidentiality of IRB/IEC Information}

The Sponsor is required to record the name and address of each IRB/IEC member that reviews and approves this trial. The Sponsor is also required to document that each IRB/IEC meets regulatory and ICH GCP requirements by requesting and maintaining records of the names and qualifications of the IRB/IEC members and to make these records available for regulatory agency review upon request by those agencies.

\subsection{Compliance with Financial Disclosure Requirements}

Financial Disclosure requirements are outlined in the US Food and Drug Administration Regulations, Financial Disclosure by Clinical Investigators (21 CFR Part 54). It is the Sponsor's responsibility to determine, based on these regulations, whether a request for 
Financial Disclosure information is required. It is the investigator's/subinvestigator's responsibility to comply with any such request.

The investigator/subinvestigator(s) agree, if requested by the Spon sor in accordance with 21 CFR Part 54, to provide his/her financial interests in and/or arrangements with the Sponsor to allow for the submission of complete and accurate certification and disclosure statements. The investigator/subinvestigator(s) further agree to provide this information on a Certification/Disclosure Form, commonly known as a financial disclosure form, provided by the Sponsor or through a secure password-protected electronic portal provided by the Sponsor. The investigator/subinvestigator(s) also consent to the transmission of this information to the Sponsor in the United States for these purposes. This may involve the transmission of information to countries that do not have laws protecting personal data.

\subsection{Compliance with Law, Audit and Debarment}

By signing this protocol, the investigator agrees to conduct the trial in an efficient and diligent manner and in conformance with this protocol; generally accepted standards of Good Clinical Practice (e.g., International Conference on Harmonization of Technical Requirements for Registration of Pharmaceuticals for Human Use Good Clinical Practice: Consolidated Guideline and other generally accepted standards of good clinical practice); and all applicable federal, state and local laws, rules and regulations relating to the conduct of the clinical trial.

The Code of Conduct, a collection of goals and considerations that govern the ethical and scientific conduct of clinical investigations sponsored by Merck, is provided in Section 12.1 Merck Code of Conduct for Clinical Trials.

The investigator also agrees to allow monitoring, audits, IRB/ERC review and regulatory authority inspection of trial-related documents and procedures and provide for direct access to all trial-related source data and documents.

The investigator agrees not to seek reimbursement from subjects, their insurance providers or from government programs for procedures included as part of the trial reimbursed to the investigator by the Sponsor.

The investigator shall prepare and maintain complete and accurate trial documentation in compliance with Good Clinical Practice standards and applicable federal, state and local laws, rules and regulations; and, for each subject participating in the trial, provide all data, and, upon completion or termination of the clinical trial, submit any other reports to the Sponsor as required by this protocol or as otherwise required pursuant to any agreement with the Sponsor.

Trial documentation will be promptly and fully disclosed to the Sponsor by the investigator upon request and also shall be made available at the trial site upon request for inspection, copying, review and audit at reasonable times by representatives of the Sponsor or any regulatory authorities. The investigator agrees to promptly take any reasonable steps that are 
requested by the Sponsor as a result of an audit to cure deficiencies in the trial documentation and worksheets/case report forms.

The investigator must maintain copies of all documentation and records relating to the conduct of the trial in compliance with all applicable legal and regulatory requirements. This documentation includes, but is not limited to, the protocol, worksheets/case report forms, advertising for subject participation, adverse event reports, subject source data, correspondence with regulatory authorities and IRBs/ERCs, consent forms, investigator's curricula vitae, monitor visit logs, laboratory reference ranges, laboratory certification or quality control procedures and laboratory director curriculum vitae. By signing this protocol, the investigator agrees that documentation shall be retained until at least 2 years after the last approval of a marketing application in an ICH region or until there are no pending or contemplated marketing applications in an ICH region or until at least 2 years have elapsed since the formal discontinuation of clinical development of the investigational product. Because the clinical development and marketing application process is variable, it is anticipated that the retention period can be up to 15 years or longer after protocol database lock. The Sponsor will determine the minimum retention period and notify the investigator when documents may be destroyed. The sponsor also recognizes that documents may need to be retained for a longer period if required by local regulatory requirements. All trial documents shall be made available if required by relevant regulatory authorities. The investigator must consult with and obtain written approval by the Sponsor prior to discarding trial and/or subject files.

ICH Good Clinical Practice guidelines recommend that the investigator inform the subject's primary physician about the subject's participation in the trial if the subject has a primary physician and if the subject agrees to the primary physician being informed.

The investigator will promptly inform the Sponsor of any regulatory authority inspection conducted for this trial.

Persons debarred from conducting or working on clinical trials by any court or regulatory authority will not be allowed to conduct or work on this Sponsor's trials. The investigator will immediately disclose in writing to the Sponsor if any person who is involved in conducting the trial is debarred or if any proceeding for debarment is pending or, to the best of the investigator's knowledge, threatened.

In the event the Sponsor prematurely terminates a particular trial site, the Sponsor will promptly notify that trial site's IRB/IEC.

According to European legislation, a Sponsor must designate an overall coordinating investigator for a multi-center trial (including multinational). When more than one trial site is open in an EU country, Merck, as the Sponsor, will designate, per country, a national principal coordinator (Protocol CI), responsible for coordinating the work of the principal investigators at the different trial sites in that Member State, according to national regulations. For a single-center trial, the Protocol CI is the principal investigator. In addition, the Sponsor must designate a principal or coordinating investigator to review the 
trial report that summarizes the trial results and confirm that, to the best of his/her knowledge, the report accurately describes the conduct and results of the trial [Clinical Study Report (CSR) CI]. The Sponsor may consider one or more factors in the selection of the individual to serve as the Protocol CI and or CSR CI (e.g., availability of the CI during the anticipated review process, thorough understanding of clinical trial methods, appropriate enrollment of subject cohort, timely achievement of trial milestones). The Protocol CI must be a participating trial investigator.

\subsection{Compliance with Trial Registration and Results Posting Requirements}

Under the terms of the Food and Drug Administration Modernization Act (FDAMA) and the Food and Drug Administration Amendments Act (FDAAA), the Sponsor of the trial is solely responsible for determining whether the trial and its results are subject to the requirements for submission to the Clinical Trials Data Bank, http://www.clinicaltrials.gov. Merck, as Sponsor of this trial, will review this protocol and submit the information necessary to fulfill these requirements. Merck entries are not limited to FDAMA/FDAAA mandated trials. Information posted will allow subjects to identify potentially appropriate trials for their disease conditions and pursue participation by calling a central contact number for further information on appropriate trial locations and trial site contact information.

By signing this protocol, the investigator acknowledges that the statutory obligations under FDAMA/FDAAA are that of the Sponsor and agrees not to submit any information about this trial or its results to the Clinical Trials Data Bank.

\subsection{Quality Management System}

By signing this protocol, the Sponsor agrees to be responsible for implementing and maintaining a quality management system with written development procedures and functional area standard operating procedures (SOPs) to ensure that trials are conducted and data are generated, documented, and reported in compliance with the protocol, accepted standards of Good Clinical Practice, and all applicable federal, state, and local laws, rules and regulations relating to the conduct of the clinical trial.

\subsection{Data Management}

The investigator or qualified designee is responsible for recording and verifying the accuracy of subject data. By signing this protocol, the investigator acknowledges that his/her electronic signature is the legally binding equivalent of a written signature. By entering his/her electronic signature, the investigator confirms that all recorded data have been verified as accurate.

Detailed information regarding Data Management procedures for this protocol will be provided separately. 


\subsection{Publications}

This trial is intended for publication, even if terminated prematurely. Publication may include any or all of the following: posting of a synopsis online, abstract and/or presentation at a scientific conference, or publication of a full manuscript. The Sponsor will work with the authors to submit a manuscript describing trial results within 12 months after the last data become available, which may take up to several months after the last subject visit in some cases such as vaccine trials. However, manuscript submission timelines may be extended on OTC trials. For trials intended for pediatric-related regulatory filings, the investigator agrees to delay publication of the trial results until the Sponsor notifies the investigator that all relevant regulatory authority decisions on the trial drug have been made with regard to pediatric-related regulatory filings. Merck will post a synopsis of trial results for approved products on www.clinicaltrials.gov by 12 months after the last subject's last visit for the primary outcome, 12 months after the decision to discontinue development, or product marketing (dispensed, administered, delivered or promoted), whichever is later.

These timelines may be extended for products that are not yet marketed, if additional time is needed for analysis, to protect intellectual property, or to comply with confidentiality agreements with other parties. Authors of the primary results manuscript will be provided the complete results from the Clinical Study Report, subject to the confidentiality agreement. When a manuscript is submitted to a biomedical journal, the Sponsor's policy is to also include the protocol and statistical analysis plan to facilitate the peer and editorial review of the manuscript. If the manuscript is subsequently accepted for publication, the Sponsor will allow the journal, if it so desires, to post on its website the key sections of the protocol that are relevant to evaluating the trial, specifically those sections describing the trial objectives and hypotheses, the subject inclusion and exclusion criteria, the trial design and procedures, the efficacy and safety measures, the statistical analysis plan, and any amendments relating to those sections. The Sponsor reserves the right to redact proprietary information.

For multicenter trials, subsequent to the multicenter publication (or after public disclosure of the results online at www.clinicaltrials.gov if a multicenter manuscript is not planned), an investigator and his/her colleagues may publish their data independently. In most cases, publication of individual trial site data does not add value to complete multicenter results, due to statistical concerns. In rare cases, publication of single trial site data prior to the main paper may be of value. Limitations of single trial site observations in a multicenter trial should always be described in such a manuscript.

Authorship credit should be based on 1) substantial contributions to conception and design, or acquisition of data, or analysis and interpretation of data; 2) drafting the a rticle or revising it critically for important intellectual content; and 3) final approval of the version to be published. Authors must meet conditions 1,2 and 3. Significant contributions to trial execution may also be taken into account to determine authorship, provided that contributions have also been made to all three of the preceding authorship criteria. Although publication planning may begin before conducting the trial, final decisions on authorship and the order of authors' names will be made based on participation and actual contributions to 
the trial and writing, as discussed above. The first author is responsible for defending the integrity of the data, method(s) of data analysis and the scientific content of the manuscript.

The Sponsor must have the opportunity to review all proposed abstracts, manuscripts or presentations regarding this trial 45 days prior to submission for publication/presentation. Any information identified by the Sponsor as confidential must be deleted prior to submission; this confidentiality does not include efficacy and safety results. Sponsor review can be expedited to meet publication timelines.

\subsection{LIST OF REFERENCES}

1. Report of a WHO Consultation Organized in Collaboration with the Viral Hepatitis Prevention Board. Global surveillance and control of hepatitis C. J of Hepatology 1999; 6:35-47.

2. Leung, NWY. Management of viral hepatitis C. Gastroenterology-Hepatology. 2002;17: S146- S154.

3. Poordad F, McCone J Jr, Bacon BR, Bruno S, Manns MP, Sulkowski MS, Jacobson IM, Reddy KR, Goodman ZD, Boparai N, DiNubile MJ, Sniukiene V, Brass CA, Albrecht JK, Bronowicki JP; SPRINT-2 Investigators. Boceprevir for untreated chronic HCV genotype 1 infection. N Engl J Med. 2011; 364:1195-206.

4. McHutchison JG, Everson GT., Gordon SC, Jacobson IM: Sulkowski M, Kauffman R, McNair L, Alam J, Muir AJ., PROV. Telaprevir with peginterferon and ribavirin for chronic HCV genotype 1 infection. N. Engl. J Med. 2009; 360 (18): 1827- 1838.

5. Armstrong GL, Wasley A, Simard EP, McQuillan GM, Kuhnert WL, Alter MJ. The prevalence of Hepatitis C virus infection in the United States, 1999 through 2002. Ann Intern Med 2006;144(10):705-14.

6. Finelli L, Miller JT, Tokars JI, Alter MJ, Arduino MJ. National surveillance of dialysisassociated diseases in the United States. Semin Dialysis 2002;18(1):52-61.

7. Fissell RB, Bragg-Gresham JL, Woods JD, Jadoul M, Gillespie B, Hedderwick SA, et al. Patterns of hepatitis $\mathrm{C}$ prevalence and seroconversion in hemodialysis units from three continents: The DOPPS. Kidney Int 2004;65:2335-42.

8. Coresh J, Astor BC, Greene T, Eknoyan G, Levey AS. Prevalence of chronic kidney disease and decreased kidney function in the adult US Population: Third national health and nutrition examination survey. Am J Kidney Dis 2003;41(1):1-12.

9. Satapathy SK, Lingisetty CS, Williams S. Higher prevalence of chronic kidney disease and shorter renal survival in patients with chronic hepatitis $C$ virus infection. Hepatol Int 2012;6:369-78. 
Protocol/Amendment No.: 052-04

10. Crook ED, Penumalee S, Gavini B, Filippova K: Hepatitis C is a predictor of poorer renal survival in diabetic patients. Diabetes Care 2005;28:2187-2191.

11. Butt AA, Wang X, Fried LF. HCV Infection and the incidence of CKD. Am J Kidney Dis 2011;57(3):396-402.

12. Noureddine LA, Usman SA, Yu Z, Moorthi RN, Moe SM. Hepatitis C increases the risk of progression of chronic kidney disease in patients with Glomerulonephritis. J Nephrol 2010;32:311-6.

13. El-Serrag HB, Hampel H, Yeh C, Rabeneck L. Extrahepatic manifestations of Hepatitis C among United States male vererans. Hepatology 2002;36(6):1440-6.

14. Fabrizi F, Aghemo A, Messa P. Impact of hepatitis $C$ on survivial of dialysis patients: a link with cardiovascular mortality? J Viral Hepat Int 2013:1-6.

15. Fabrizi F, Martin P, Dixit V, Bunnapradist S, Kanwal F, Dulai G. Post-transplant diabetes mellitus and HCV seropositive status after renal transplantation: meta-analysis of clinical studies. Am J Transplant 2005;5:2433-40.

16. Rocatello D, Fornasieri A, Giachino O, Rossi D, Beltrame A, Banfi G, et al. Multicenter study on Hepatitis C virus-related Cryoglobulinemic Glomerulonephritis. Am J Kidney Dis 2013;49(1):69-82.

17. Cruzado JM, Casanovas-Taltavull T, Torras J, Baliellas C, Gil-Vernet S, Grinyo JM. Pretransplant interferon prevents Hepatitis C virus-associated Glomerulonephritis in renal allografts by NCA-RNA clearance. Am J Transplant 2003;3:357-60.

18. Fabrizi F, Martin P, Dixit V, Bunnapradist S, Dulai G. Hepatitis C virus antibody status and survival after renal transplantation: Meta-analysis of observational studies. Am J Transplant 2005;5:1452-61.

19. Gordon CE, Balk EM, Becker BN, Crooks PA, Jaber BL, Johnson CA, et al. KDOQI US commentary on the KDIGO clinical practice guideline for the prevention, diagnosis, evaluation, and treatment of Hepatitis C in CKD. Am J Kidney Dis 2008;52(5):811-25.

20. Fabrizi F, Dixit V, Messa P, Martin P. Interferon monotherapy of chronic hepatitis C in dialysis patients: meta-analysis of clinical trials. J Viral Hepat 2008;15:79-88.

21. Fabrizi F, Dixit V, Messa P, Martin P. Pegylated interferon monotherapy of chronic hepatitis $\mathrm{C}$ in dialysis patients: meta-analysis of clinical trials. J Viral Hepat 2010;82:768-75.

22. Fabrizi F, Dixit V, Martin P, Messa P. Combined antiviral therapy of hepatitis C virus in dialysis patients: meta-analysis of clinical trials. J Viral Hepat 2011;18:e263-e269. 
Protocol/Amendment No.: 052-04

23. Basu PP, Siriki R, Shah NJ, Farhat S, Mittimana K, Atluri S, et al. Telaprevir with adjusted dose of ribavirin in naïve CHC-G1: Efficacy and treatment in CHC in hemodialysis population. Target C (RCT). J Hepatol 2013;58:S25-S44.

24. Mauss S, Hueppe D, Alshuth U. Renal impairment is frequent in chronic hepatitis C patients under triple therapy with telaprevir or boceprevir.

25. Virlogeux V, Goncalves F, Pradat P, Maynard M, Hartig-Lavie K, Amiri M, et al. Boceprevir and telaprevir based triple therapy for chronic hepatitis C: On-treatment efficacy and impact on kidney function and meld score. J of Hepatol 2013;58:S229-S407.

26. Alberti A. Impact of a sustained virological response on the long-term outcome of hepatitis C. Liver Int. 2011 Jan;31 Suppl 1:18-22.

27. Martinot-Peignoux M, Stern C, Maylin S, Ripault MP, Boyer N, Leclere L, Castelnau C, Giuily N, El Ray A, Cardoso AC, Moucari R, Asselah T, Marcellin P. Twelve weeks posttreatment follow-up is as relevant as 24 weeks to determine the sustained virologic response in patients with hepatitis $C$ virus receiving pegylated interferon and ribavirin. Hepatology. 2010 Apr;51(4):1122-6.

28. McHutchison JG, Lawitz EJ, Shiffman ML, et al. Peginterferon alfa-2b or alfa-2a with ribavirin for treatment of hepatitis C infection. N Engl J Med 2009; 361(6):580-93.

29. Miettinen O, Nurminen M. Comparative analysis of two rates. Statistics in Medicine 1985; 4: 213-26.

30. Koch GG, Johnson WD, and Tolley HD. A linear models approach to the analysis of survival and extent of disease in multidimensional contingency tables. Journal of the American Statistical Association 1972; 67(340):783-796.

31. Zhao Y, Preisser JS, and Koch GG. Sensitivity Analysis for Withdrawals in Grouped Time-to-event Data. Statistics in Biopharmaceutical Research, Published online: 06 Sep 2013 URL http://www.tandfonline.com/eprint/itd4wGeQzz45muHk6x6V/full

32. Spiegel BMR, Younossi ZM, Hays RD, Revicki D, Robbins S, Kanwal F. Impact of hepatitis $\mathrm{C}$ on health related quality of life: a systematic review and quantitative assessment. Hepatology 2005; 41:790-800.

33. Foster GR. Quality of life considerations for patients with chronic hepatitis C. Journal of Viral Hepatitis 2009; 16:605-611.

34. Fabrizi F, Messa P, Martin P. Health-related quality of life in dialysis patients with HCV infection. Int J Artif Organs 2009; 32:473-81.

35. Avramovic M, Stefanovic V. Health-related quality of life in different stages of renal failure. Artificial Organs 2012; 36(7):581-589. 
Protocol/Amendment No.: 052-04

36. Mujais SK, Story K, Brouillette J, Takano T, Soroka S, Franek C, Mendelssohn D and Finkelstein FO. Health-related quality of life in CKD patients: correlates and evolution over time. Clin J Am Soc Nephrol 2009; 4: 1293-1301.

37. Molsted S, Prescott L, Heaf J, et al. Assessment and clinical aspects of health-related quality of life in dialysis patients and patients with chronic kidney disease. Nephron Clin Pract 2007; 106(1):c24-33.

38. Soni RK, Weisbord SD, Unruh ML. Health-related quality of life outcomes in chronic kidney disease. Curr Opin Nephrol Hypertens 2010; 19(2):153-159.

39. Y Zhang, L Zhang, S Abraham, et al. Assessment of the Impact of Renal Impairment on Systemic Exposure of New Molecular Entities: Evaluation of Recent New Drug Applications. Clin Pharmacol Ther. 2009 Mar;85(3):305-11. doi: 10.1038/clpt.2008.208. Epub 2008 Nov 19.

40. TW Lapinski, A Parfieniuk, M Rogalska-Plonska, et al. Prevalence of cryoglobulinaemia in hepatitis C virus- and hepatitis C virus/human immunodeficiency virus-infected individuals: implications for renal function. Liver International 2009; ISSN 1478-3223: 1158-1161.

41. Foucher J, Chanteloup E, Vergniol J, Caste'ra L, Le Bail B, Adhoute X, Bertet J, Couzigou P, de Le'dinghen V. Diagnosis of cirrhosis by transient elastography (FibroScan): a prospective study. Gut 2006;55:403-408.

42. Castera L, Forns X, Alberti A. Non-Invasive evaluation of liver fibrosis using transient elastography. Journal of Hepatology 2008; 48: 835-847.

43. Boursier J, de Ledinghen V, Zarski JP, Fouchard-Hubert I, Gallois Y, Overti F, Cales P and multicentric groups from SNIFF 32, VINDIAG 7, and ANRS/HC/EP23 FIRBOSTAR studies. Hepatology 2012; 55(1): 58-67.

44. Chou R, Wasson N. Blood tests to diagnose fibrosis or cirrhosis in patients with chronic hepatitis C virus infection. Annals of Internal Medicine 2013;158:807-820. 